\title{
WEEDS OF WHEAT IN NEPAL: A LITERATURE REVIEW
}

\author{
D. R. Dangol
}

\section{ABSTRACT}

This paper reviews publications dealing with weeds and/or their control in wheat (Triticum aestivum L.) in Nepal and enumerates 370 weed species of 210 genera in 54 families. This review shows that the Asteraceae (69 spp.), Poaceae (52 spp.), Leguminosae (25 spp.), Polygonaceae (19 spp.), Caryophyllaceae (18 spp.), Scrophulariaceae (17 spp.), Euphorbiaceae and Lamiaceae (14 spp. each), Brassicaceae and Cyperaceae (13 spp.), and Solanaceae (11 spp. each) were the dominant families, which accounted for $71.35 \%$ of the total weeds. This paper also documented 63 species reported as the principal or most common weeds and 4 species (Chenopodium album, Cynodon dactylon, Phalaris minor, Polygonum plebeium) as the most commonly reported weeds of wheat in Nepal in the papers reviewed. This paper also highlighted the fact that the researchers from the Tribhuvan University, Department of Plant Resources (then Department of Medicinal Plants/HMG) and Nepal Agricultural Research Council mentioned about the deposition of their herbarium specimens in the respective organizations. This paper also analyzed the publication media in which the reviewed papers appeared.

Key words: principal weeds, Triticum aestivum, weed flora, weed survey, wheat

\section{INTRODUCTION}

In Nepal, wheat (Triticum aestivum L.) is the principal winter crop with an annual production of 1746 thousand M. Tons in 2011 (www.indexmundi.com). The average yield of this crop is $1300 \mathrm{Kg} / \mathrm{ha}$ which is low in comparison to other countries. The low production of wheat may be associated with many factors; among them, weeds are one of the major problems. The weeds infesting the wheat crop reduce the crop yield upto $50 \%$ in Nepal (Malla and Ranjit 1980, Ranjit 1982, Bhatta 1981, Shivakoti et al. 1977). To get maximum production, weeds of wheat should be managed properly. Since the weed management depends upon the type and intensity of weeds present in the crop fields, a sound knowledge on wheat weeds is necessary for determining economic control measures (Holm et al. 1977, Gupta 1984). With the same viewpoints, weeds of wheat fields from different parts of Nepal were reported (See references). However, these reports remain scattered up-to-date. This study was, therefore, undertaken to review the literature to prepare a list of species, determine the principal weed species and their distribution and herbarium specimen records mentioned in the papers dealing with weeds of wheat and their control in Nepal.

\section{TRENDS IN WEED SURVEY IN WHEAT FIELDS}

There are altogether 26 published papers on weeds and their control: 9 dealing with taxonomy and some aspects of weed flora, 8 with weed control on wheat and 2 with weed flora and their control. Numata, a Japanese ecologist published a paper on "methodology for the study of weed vegetation" giving examples of weeds of wheat of Nepal (Numata 1982). The studies on weeds and /or their control was initiated by Dr. O. P. Gupta in 1977 who worked as a visiting professor at the Institute of Agriculture and Animal Science, Tribhuvan University, Rampur, 
Chitwan, Nepal for 2 years. In late 1970's the results of weed surveys in crop fields of Rampur (Gupta et al. 1977) and Kathamndu Valley (Rajbhandary and Ojha 1979) were reported. In 1980's, there were some works on weed flora of Jiri (Numata 1982), Kavre (Joshi and Gretzmacher 1999), Birganj (Dangol 1987), Rampur (Dangol et al. 1988, Shah et al. 2011ab), Kirtipur, Kathmandu (Chaudhary and Shrestha 1981), Khokana, Lalitpur (Sapkota et al. 2010) and Nepal (Regmi et al. 1986). Research works on weed control trials were also conducted in different Agricultural Research Stations of Nepal (Bhatta 1981, Malla and Ranjit 1980, Ranjit 81/82, 1982, 1983a, 1983b, Sthapit 1986). Mazek (1991) studied the weed vegetation of cultivated fields in Nepal. There are two reports that deal with weed and weed control aspect. The summary of the family, genera and species along with study locations mentioned in the papers reviewed is given in table 1.

Table 1. List of the number of family (F), genus (G) and species (S) and study areas of the wheat weeds reported by the authors in the papers reviewed.

\begin{tabular}{|c|c|c|c|c|c|c|}
\hline SN & Authors \# & $\mathbf{F}$ & G & $\mathbf{S}$ & Study area* & Remarks** \\
\hline 1. & Bhatta (1981) & 3 & 6 & 4 & Bhairahawa & Trifolium, Vicia \\
\hline 2. & Chaudhary $(1979)^{\mathrm{C}}$ & 36 & 83 & 108 & $\begin{array}{l}\text { Kathmandu } \\
\text { valley }\end{array}$ & \\
\hline 3. & $\begin{array}{l}\text { Chaudhary and } \\
\text { Shrestha (1981) }\end{array}$ & 4 & 4 & 5 & Kirtipur & \\
\hline 4. & Dangol (1987)' & 16 & 33 & 35 & Birganj & \\
\hline 5. & Dangol (2000-2001)' & 21 & 54 & 65 & $\begin{array}{l}\text { Inner Terai } \\
\text { and Terai }\end{array}$ & \\
\hline 6. & Dangol et al. (1988)' & 27 & 57 & 74 & Rampur areas & \\
\hline 7. & $\begin{array}{l}\text { Dangol and Chaudhary } \\
\text { (1991) }\end{array}$ & 16 & 23 & 30 & Rampur & $\begin{array}{l}\text { Ixeris, } \\
\text { Unidentified } \\
\text { genus }\end{array}$ \\
\hline 8. & Dangol (2001) & 18 & 27 & 31 & Rampur & \\
\hline 9. & Devkota (1983) & 11 & 17 & 14 & & $\begin{array}{l}\text { Melilotus, Rumex, } \\
\text { Vicia, }\end{array}$ \\
\hline 10. & Gupta et al. $(1977)^{A}$ & 9 & 9 & 9 & Rampur & \\
\hline 11. & $\begin{array}{l}\text { Joshi and Gretzmacher } \\
(1999)\end{array}$ & 22 & 48 & 64 & Kavre & \\
\hline 12. & Malla and Ranjit (1980) & 5 & 6 & 4 & Khumal & Cyperus, Vicia, \\
\hline 13. & Manandhar $(1978)^{\mathrm{N}}$ & 19 & 86 & 122 & $\begin{array}{l}\text { Kathmandu } \\
\text { valley }\end{array}$ & \\
\hline
\end{tabular}


J. Nat. Hist. Mus. Vol. 27, 2013

\begin{tabular}{|c|c|c|c|c|c|c|}
\hline 14. & Mazek (1991) & 31 & 82 & 113 & Nepal & \\
\hline 15. & Numata (1982) & 7 & 9 & 11 & Jiri & Polygonum \\
\hline 16. & $\begin{array}{l}\text { Rajbhandary and Ojha } \\
\text { (1979) }\end{array}$ & 20 & 34 & 39 & $\begin{array}{l}\text { Kathmandu } \\
\text { valley }\end{array}$ & Heliotropium \\
\hline 17. & Ranjit (1981/82) & 4 & 7 & 5 & $\begin{array}{l}\text { Khu, Bhw and } \\
\text { Har }\end{array}$ & Rumex, Trifolium \\
\hline 18. & Ranjit (1983a) & 7 & 14 & 15 & Khumal & \\
\hline 19. & Ranjit (1983b) & 6 & 11 & 8 & $\begin{array}{l}\text { Khu, Bhw and } \\
\text { Har }\end{array}$ & $\begin{array}{l}\text { Alopecurus, } \\
\text { Rumex, Trifolium }\end{array}$ \\
\hline 20. & Ranjit (1999) & 6 & 11 & 7 & & $\begin{array}{l}\text { Alepecurus, } \\
\text { Gnaphalium, } \\
\text { Polygonum }\end{array}$ \\
\hline 21. & Ranjit (2003) & 6 & 8 & 6 & & $\begin{array}{l}\text { Alopecurus, } \\
\text { Rumex }\end{array}$ \\
\hline 22. & $\begin{array}{l}\text { Ranjit and Bhattarai } \\
\text { (1988) }\end{array}$ & 11 & 21 & 22 & Nepal & \\
\hline 23. & $\begin{array}{l}\text { Ranjit and } \\
\text { Suwanketnikom (2003) }\end{array}$ & 13 & 23 & 22 & Khumal & $\begin{array}{l}\text { Bothriospermum, } \\
\text { Lactuca, Mazus, } \\
\text { Polygonum, } \\
\text { Ranunculus }\end{array}$ \\
\hline 24. & Ranjit et al. (2006) & 20 & 48 & 43 & Nepal & $\begin{array}{l}\text { Alopecuros, } \\
\text { Alternanthera, } \\
\text { Cyperus, Digitaria, } \\
\text { Equisetum, } \\
\text { Lactuca, Lindernia, } \\
\text { Mazus, Polygonum, } \\
\text { Rumex }\end{array}$ \\
\hline 25. & Ranjit et al. (2009) & 6 & 8 & 8 & Khumal & \\
\hline 26. & Regmi et al. (1986) & 31 & 71 & 96 & Nepal & $\begin{array}{l}\text { Aster, Pratia, } \\
\text { Impatiens, Mazus, } \\
\text { Cerastium, } \\
\text { Scrophularia }\end{array}$ \\
\hline
\end{tabular}




\begin{tabular}{|c|c|c|c|c|c|c|}
\hline 27. & Sapkota et al. (2010) & 18 & 38 & 44 & Khokana & $\begin{array}{l}\text { Arenaria, Bidens, } \\
\text { Clerodendrum, } \\
\text { Convolvulus, } \\
\text { Cynoglossum, } \\
\text { Persicaria, } \\
\text { Torenia, Vernonia }\end{array}$ \\
\hline 28. & Shah et al. (2011a) & 11 & 19 & 19 & Rampur & Erigeron, Vicia \\
\hline 29. & Shah et al. (2011b) & 11 & 19 & 19 & Rampur & Erigeron, Vicia \\
\hline 30. & Sharma et al. (2010) & 19 & 38 & 43 & Manang & $\begin{array}{l}\text { Campanula, } \\
\text { Cremanthodium }\end{array}$ \\
\hline 31. & Shivakoti et al. (1977) & 3 & 3 & 3 & Rampur & \\
\hline 32. & Sthapit (1986) & 5 & 5 & 5 & LAC & \\
\hline 33. & Thapa (2003) & 26 & 48 & 57 & Pokhara & $\begin{array}{l}\text { Bothriochloa, } \\
\text { Erigeron }\end{array}$ \\
\hline 34. & $\begin{array}{l}\text { Thapa and Kayastha } \\
\text { (1996) }\end{array}$ & 26 & 48 & 54 & Pokhara & $\begin{array}{l}\text { Bothriochloa, } \\
\text { Equisetum, } \\
\text { Erigeron, } \\
\text { Marchantia, } \\
\text { Sonchus }\end{array}$ \\
\hline
\end{tabular}

\# Author(s) name marked with letters deposited their herbarium collections in different institutions in Nepal,

$A=$ Department of Agronomy, IAAS, Rampur, $C=$ Central Department of Botany (Kirtipur), $\mathrm{I}=\mathrm{IAAS}$ Herbarium (Rampur), and N=National Herbarium (Godawary),

* Khu, Bhw and Har=Khumal, Bhairahawa and Hardinath, LAC=Lumle Agricultureal Centre.

** Identified upto genus level only

\section{WEED DIVERSITY}

In Nepal, 370 species of wheat weeds representing 54 families and 210 genera of vascular plants were listed (Appendix 1). There were 4 non-flowering plants and 330 flowering plants (293 flowering plant dicots and 73 flowering plant monocots) (Figure 1). Among the total families, 16 families include 5 or more species which accounted for $79.19 \%$ of the total weed species recorded. Two most dominant families were the Aseraceae and Poaceae which had 69 and 52 weed species, respectively. There were 22 families with 2 to 4 species and 16 families with 1 species each. In the papers reviewed, the best-represented genus was Polygonum (15 spp.) followed by Euphorbia and Stellaria (8 spp. each), Eragrostis, and Solanum (7 spp. each), Vicia (6 spp.), and Lindernia and Veronica (5 spp. each). There were 15 genera with 4 spp., 16 genera with 3 spp., and 30 genera with 2 spp. The rest 141 genera were represented with $1 \mathrm{sp}$. each in the weeds of wheat in Nepal. 
J. Nat. Hist. Mus. Vol. 27, 2013

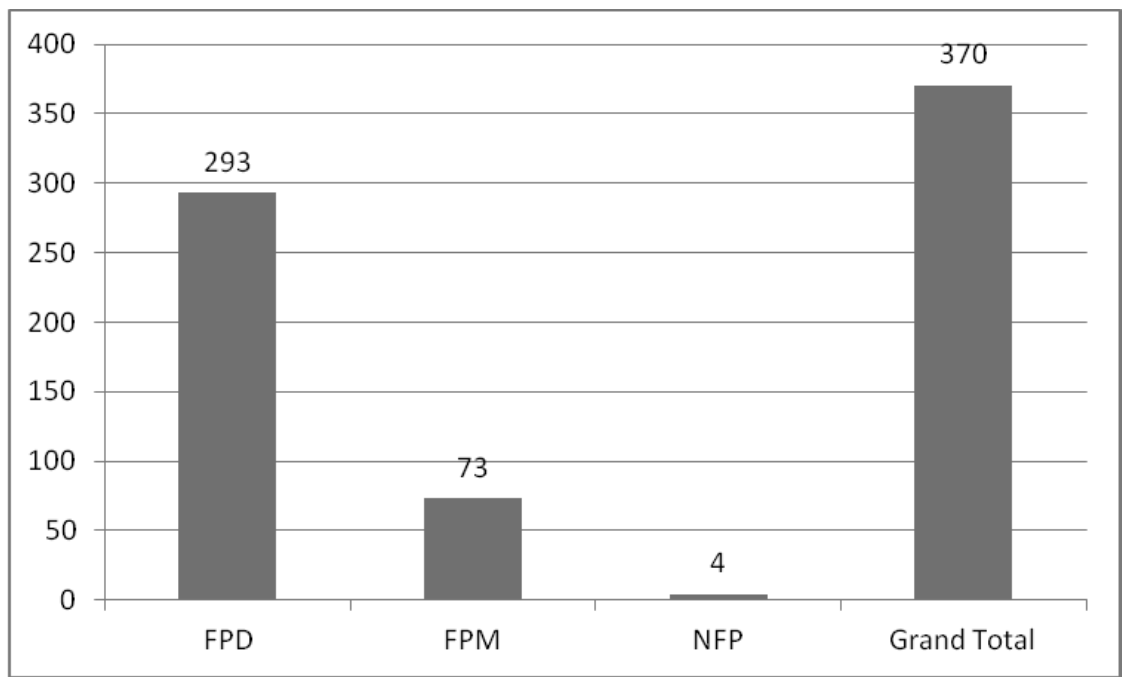

Figure 1. Floristic analysis of weed species of wheat in different groups (FPD= flowering plant dicot, FPM= flowering plants monocot, NFP= Non-flowering plants).

\section{PRINCIPAL WEEDS AND THEIR DISTRIBUTION}

Almost all authors of the reviewed papers mentioned some species as major/principal/ important weeds of wheat on the basis of visual estimation. However, Dangol et al. (1988) used summed dominance ratio $\{S D R=$ (relative density and relative frequency) $/ 2\}$ to calculate major/minor weeds. Numata (1982) also suggested such type of SDR expression for determining major weeds in farmlands. Mazek (1991) followed Braun-Blanquet method for estimating the cover value of weed species. Some authors used other ecological parameters for identifying dominant weeds (Dangol 2001, Ranjit et al. 2006, Sharma et al. 2010, Sapkota et al. 2010). The 63 weed species, which were reported as dominant/principal/abundant or major wheat weeds in the literature reviewed, are listed alphabetically in Table 2. Of the 63 major weeds, 53 were reported from hilly regions, 14 from the Inner Terai and 12 from Terai regions of Nepal. The species like Chenopodium album (Bethe), Phalaris minor (Ragate jhar), and Cynodon dactylon (Dubo), Polygonum plebeium (Sukuljhar) and Vicia sativa (Kutilkosha) were mentioned as major weeds for the three wheat growing regions of the country (table 2). Some of the weeds such as Chenopodium album, Phalaris minor, Vicia sativa, Polygonum plebeium (Sukule jhar) were the principal problem weeds of wheat in the Terai (Bhairahawa and Birganj) and hilly (Kathmandu Valley) regions of Nepal. Of the above three weeds, Chenopodium album and Cynodon dactylon were not reported as the major weeds of wheat in western hilly area (Sthapit 1986) and Phalaris minor in the central Inner Terai (Dangol et al., 1988). Some of the weeds were locally important; for example Cannabis sativa (Ganja or Bhang) was reported as one of the most problematic weeds in wheat in the Kathmandu Valley. 
Table 2. Alphabetical list of principal weeds reported in wheat of hills, inner Terai and Terai in Nepal.

\begin{tabular}{|c|c|c|c|c|c|c|c|c|c|}
\hline SN & Principal species & Hills $^{1}$ & $\begin{array}{l}\text { Inner } \\
\text { Terai }^{2} \\
\end{array}$ & Terai $^{3}$ & SN & Principal species & Hills $^{1}$ & $\begin{array}{l}\text { Inner } \\
\text { Terai }^{2} \\
\end{array}$ & Terai $^{3}$ \\
\hline 1. & Ageratum conyzoides & $\mathrm{K}$ & & & 33 & Lolium temulentum & & & $\mathrm{B}$ \\
\hline 2. & $\begin{array}{l}\text { Ageratum } \\
\text { houstonianum }\end{array}$ & & $\mathrm{C}$ & & 34 & Mazus japonicus & $\mathrm{K}$ & $\mathrm{C}$ & \\
\hline 3. & Alopecurus aequalis & $\mathrm{K}$ & & & 35 & Medicago denticulata & $\mathrm{P}$ & $\mathrm{C}$ & $\mathrm{R}$ \\
\hline 4. & $\begin{array}{l}\text { Alopecurus } \\
\text { nepalensis }\end{array}$ & $\mathrm{K}$ & & & 36 & Melilotus alba & & & $\mathrm{R}$ \\
\hline 5. & Alopecurus pratensis & $\mathrm{N}$ & & & 37 & Oxalis corniculata & $\mathrm{K}$ & C & \\
\hline 6. & Alternanthera sessilis & $\mathrm{K}$ & & & 38 & Oxalis latifolia & $\mathrm{K}$ & & \\
\hline 7. & Anagallis arvensis & & $\mathrm{C}$ & $\mathrm{B}, \mathrm{R}$ & 39 & Paspalum distichum & $\mathrm{J}$ & & \\
\hline 8. & Aeschynomene indica & $\mathrm{J}$ & & & 40 & Phalaris minor & $\mathrm{K}, \mathrm{L}, \mathrm{N}, \mathrm{P}$ & $\mathrm{C}$ & $\mathrm{B}, \mathrm{H}, \mathrm{R}$ \\
\hline 9. & Bidens pilosa & $\mathrm{P}$ & & & 41 & Phleum alpinum & $M$ & & \\
\hline 10. & $\begin{array}{l}\text { Botrhiospermum } \\
\text { tenellum }\end{array}$ & & $\mathrm{C}$ & & 42 & Phyla nodiflora & $\mathrm{K}$ & & \\
\hline \begin{tabular}{|l|}
11. \\
\end{tabular} & Cannabis sativa & $\mathrm{K}$ & & & 43 & Plantago major & $\mathrm{K}$ & & \\
\hline 12. & $\begin{array}{l}\text { Capsella bursa- } \\
\text { pastoris }\end{array}$ & $\mathrm{K}$ & & & 44 & Polygonum aviculare & $\mathrm{K}$ & & \\
\hline 13. & Carex nubigena & $\mathrm{J}$ & & & 45 & Polygonum capitatum & $\mathrm{L}$ & & \\
\hline 14. & Centella asiatica & $P$ & & & 46 & Polygonum hydropiper & $\mathrm{K}, \mathrm{L}, \mathrm{N}$ & & \\
\hline 15. & Chenopodium album & $\mathrm{K}, \mathrm{M}, \mathrm{N}, \mathrm{P}$ & $\mathrm{C}$ & $\mathrm{B}, \mathrm{H}, \mathrm{R}$ & 47 & Polygonum plebeium & $\mathrm{K}, \mathrm{N}, \mathrm{P}$ & $\mathrm{C}$ & $\mathrm{R}$ \\
\hline 16. & Convolvulus arvensis & $\mathrm{M}$ & & & 48 & Polygonum viscosum & $\mathrm{N}$ & & \\
\hline 17. & Coronopus didymus & $\mathrm{K}$ & & & 49 & Polypogon fugax & $\mathrm{N}$ & & \\
\hline 18. & Cynodon dactylon & $\mathrm{K}, \mathrm{N}, \mathrm{P}$ & $\mathrm{C}$ & $B, R$ & 50 & $\begin{array}{l}\text { Polypogon } \\
\text { monspeliensis }\end{array}$ & & & $\mathrm{R}$ \\
\hline 19. & Cyperus cyperoides & $\mathrm{J}$ & & & 51 & Rorippa palustris & $\mathrm{K}$ & & \\
\hline 20. & Cyperus rotundus & $P$ & & & 52 & Rumex crispus & $\mathrm{K}$ & & \\
\hline 21. & Digitaria adscendens & & $\mathrm{C}$ & & 53 & Rumex dentatus & & & $\mathrm{B}, \mathrm{R}$ \\
\hline 22. & Digitaria sanguinalis & & C & & 54 & Senecio vulgaris & $\mathrm{K}$ & & \\
\hline 23. & Drymaria cordata & $L$ & & & 55 & Sennebiera pinnatifida & $\mathrm{K}$ & & \\
\hline 24. & Eclipta prostrata & $L$ & & & 56 & Solanum carolinense & $\mathrm{J}$ & & \\
\hline 25. & Equisetum debile & $\mathrm{P}$ & & $\mathrm{B}$ & 57 & Solanum nigrum & $\mathrm{J}$ & & \\
\hline 26. & Euphorbia hirta & $\mathrm{K}$ & & & 58 & Soliva anthemifolia & $\mathrm{K}$ & & \\
\hline 27. & Fumaria indica & $\mathrm{P}$ & & & 59 & Stellaria media & $\mathrm{K}$ & & \\
\hline 28. & Galinsoga parviflora & $\mathrm{K}, \mathrm{L}$ & & & 60 & Vicia angustifolia & $\mathrm{K}$ & $\mathrm{C}$ & \\
\hline 29. & $\begin{array}{l}\text { Gnaphalium luteo- } \\
\text { album }\end{array}$ & $\mathrm{P}$ & $\mathrm{C}$ & B & 61 & Vicia hirsuta & $\mathrm{K}$ & $\mathrm{C}$ & \\
\hline 30. & $\begin{array}{l}\text { Hydrocotyle } \\
\text { nepalensis }\end{array}$ & $\mathrm{K}$ & & & 62 & Vicia sativa & $\mathrm{K}$ & $\mathrm{C}$ & $\mathrm{R}$ \\
\hline 31. & Imperata cylindrica & $\mathrm{K}$ & & & 63 & Vicia tetrasperma & $\mathrm{K}$ & & \\
\hline 32. & Lathyrus aphaca & & $\mathrm{C}$ & $\mathrm{B}, \mathrm{H}, \mathrm{R}$ & & & & & \\
\hline
\end{tabular}

${ }^{1}$ Hills: J=Jiri; $\mathrm{K}=$ Kathmandu valley; L=Lumle Agricultural Centre; $M=M a n a n g ; N=N a l d u n g ;$ $\mathrm{P}=$ Pokhara;

${ }^{2}$ Inner Terai: C=Chitwan (224 m),

${ }^{3}$ Terai: R=Rupandehi (Bhairahawa (109 m), Paklihawa, Chhotibansar); 6=Birganj (86 m); 
$7=$ Hardinath $(100 \mathrm{~m})]$ in which a particular species of weeds is present and $\mathrm{M}=$ Mangang Chenopodium album was the most commonly reported weed of wheat in Nepal which was listed in 31 papers out of 34 papers reviewed (table 3). Cynodon dactylon, Phalaris minor and Polygonum plebeium were the weeds mentioned in 28,25 , and 21 articles reviewed. Cannabis sativa and Gnaphalium luteo-album were listed in 18 papers and Oxalis corniculata was reported as weed in 15 literatures reviewed. One-hundred seventy-seven weeds were reported in only one literature (table 3). Chenopodium album and Cynodon dactylon were regarded as being the most important weeds in wheat in the world (Holm et al. 1977)

Table 3. The most commonly reported weeds of wheat in Nepal in the literature reviewed (total number of literature reviewed is 34 ).

\begin{tabular}{|c|c|c|c|}
\hline SN & Species list & $\begin{array}{r}\text { Total } \\
\text { species }\end{array}$ & $\begin{array}{r}\text { Total } \\
\text { literature }\end{array}$ \\
\hline 1. & Chenopodium album & 1 & 31 \\
\hline 2. & Cynodon dactylon & 1 & 28 \\
\hline 3. & Phalaris minor & 1 & 25 \\
\hline 4. & Polygonum plebeium & 1 & 21 \\
\hline 5. & Cannabis sativa, Gnaphalium luteo-album & 2 & 18 \\
\hline 6. & Oxalis corniculata & 1 & 15 \\
\hline 7. & $\begin{array}{l}\text { Anagallis arvensis, Centella asiatica, Polygonum } \\
\text { hydropiper, Solanum nigrum, Vicia hirsuta, Vicia sativa }\end{array}$ & 6 & 14 \\
\hline 8. & Avena fatua, Capsella bursa-pastoris, Lathyrus aphaca & 3 & 11 \\
\hline 9. & $\begin{array}{l}\text { Alternanthera sessilis, Hydrocotyle rotundifolia, Mazus } \\
\text { japonicus, Polypogon fugax, Vicia teterasperma }\end{array}$ & 5 & 10 \\
\hline 10. & $\begin{array}{l}\text { Ageratum conyzoides, Androsace rotundiflolia, Breea } \\
\text { arvnsis, Cyperus rotundus, Fumaria parviflora, Rungia } \\
\text { parviflora, Stellaria media }\end{array}$ & 7 & 9 \\
\hline 11. & $\begin{array}{l}\text { Alopecurus aequalis, Cardamine sylvatica, Gnaphalium } \\
\text { purpureum, Mazus surculosus, Medicago denticulata, } \\
\text { Melilotus alba, Melilotus indica, Phyla nodiflora, } \\
\text { Senebiera pinnatifida, Senecio vulgaris }\end{array}$ & 10 & 8 \\
\hline 12. & $\begin{array}{l}\text { Ageratum houstonianum, Bidens pilosa, Blumea lacera, } \\
\text { Convolvulus arvensis, Trifolium repens }\end{array}$ & 5 & 7 \\
\hline 13. & $\begin{array}{l}\text { Amaranthus spinosus, Bothriospermum tenellum, Digitaria } \\
\text { ciliaris, Eclipta prostrata, Emilia sonchifolia, Euphorbia } \\
\text { hirta, Galinsoga parviflora, Ixeris polycepala, Medicago } \\
\text { lupulina, Rumex dentaus, Spergula arvensis, Soliva } \\
\text { anthemifolia, Sonchus arvensis, Sonchus asper, Xanthium } \\
\text { strumarium }\end{array}$ & 15 & 6 \\
\hline 14. & See Appendix 1 & 18 & 5 \\
\hline
\end{tabular}




\begin{tabular}{|l|l|r|r|}
\hline 15. & See Appendix 1 & 17 & 4 \\
\hline 16. & See Appendix 1 & 36 & 3 \\
\hline 17. & See Appendix 1 & 64 & 2 \\
\hline 18. & See Appendix 1 & 177 & 1 \\
\hline
\end{tabular}

\section{HERBARIUM RECORDS}

The authors employed either in National Herbarium (Kathmandu) or in the academic institutions reported about the deposition of their weed collections (table 1). The herbarium specimens of weeds were housed in National Herbarium, Godawary (Manandhar 1978); IAAS Herbarium, Rampur (Dangol 1987, Dangol et al. 1988); Agronomy Department, IAAS, Rampur (Gupta et al. 1977); and Central Department of Botany, Kirtipur (Chaudhary 1979, Chaudhary and Shrestha 1981) and Weed Science Unit, Agronomy Division, NARC, Khumaltar, Lalitpur (Joshi and Gretzmacher 1999). Except the above-mentioned authors, the others did not mention in their papers whether they had prepared herbarium specimens or not.

\section{PUBLICATION MEDIA}

The authors of the papers on weed control, except Ranjit and Bhattarai (1988), who reported 22 common weeds of wheat for Nepal in Crop Weeds and Their Control) mentioned only few weed species from experimental plots of different locations of Nepal such as Khumaltar (Central midhill), Bhairahawa (western Terai), and Hardinath (central Terai). Their reports were published in "Krishi" (magazine in Nepalese language), "Wheat Research Reports", "Wheat Report", and "Wheat, Barley and Triticale Report", "Annual Reports", etc.

Researchers published their research findings related to weedflora, ecology and weed management in different scientific journals published from Nepal, India and other countries. The authors published their research articles in the "J. Inst. Agric. Anim. Sci." (Gupta et al. 1977, Dangol, 1987, Dangol et al. 1988); "J. Nat. Hist. Mus." (Chaudhary 1979, Chaudhary and Shrestha 1981), "Nepalese J. Agric." (Rajbhandary and Ojha 1979); "Bull. Bot. Surv. India” (Manandhar 1978); "Botanica Orientalis" (Sapkota et al. 2010); "Agriculture Development Journal" (Shah et al. 2011a), "International Research Journal of Applied and Basic Sciences" (Shah et al. 2011b), "Nepal Journal of Science and Technology" (Joshi and Gretzmacher 1999) and "Kasetsart J." (Ranjit and Suwanketnicom 2003). The article of Regmi et al. (1986) was published in the proceedings of winter crops workshop held at Rampur Agriculture Station, Chitwan. It is obvious from the papers (table 1) that most of the works were either conducted in the Kathmandu valley or in the Chitwan valley. The total number of weeds reported by Regmi et al. (1986) i.e. $96 \mathrm{spp}$. for Nepal is very few in compared to the number of weeds reported from the two valleys (Kathmandu: $122 \mathrm{spp}$. and Chitwan: $78 \mathrm{spp}$.). Some genera, which were not identified into species, are listed from the papers on weed control and some from the paper of Regmi et al. (1986).

\section{CONCLUSION}

It is evident from publications that weed survey works were mostly confined either to the Kathmandu Valley or to the Chitwan Valley, and were conducted more than 20 years ago in 
the former valleys, there opens a scope of conducting systematic and ecological surveys of weed community in the wheat fields of Nepal in order to understand the succession of weed community in the wheat fields vegetation and prioritize the weed management researches. Such type of survey should lead to establish weed herbarium at least in the agricultural academic and research institutions, namely in the Institute of Agriculture and Animal Science/ Tribhuvan University, University of Agriculture and Forestry, Rampur, Chitwan and National Council of Agricultural Research, Khumaltar, Nepal. The herbarium records will aid in tracing the succession of weed communities in wheat in Nepal in future and aid as teaching and research materials for students, agriculturists, botanists and ecologists. Ecophysiological study on the principal weeds of wheat need to be studied with respect to important wheat varieties grown in Nepal so that their interaction principles could be understood and applied in the weed management in wheat.

\section{ACKNOWLEDGEMENTS}

I would like to thank two peer reviewers for their comments on the previous draft of this manuscript and Rupak Dangol for typesetting.

\section{REFERENCES}

Bhatta, M.R., 1981. Weed control experiment in wheat. Wheat, Barley and Triticale. HMG, Department of Agriculture, NWDP, Siddharthnagar, Bhairahawa, Nepal, pp 163-166.

Chaudhary, R.P. and K.K. Shrestha, 1981. Weed flora of Kirtipur area (Kathmandu Valley). J. Nat. Hist. Mus., 5(1):37-48.

Chaudhary, R.P., 1979. Report on the weeds of wheat fields of Kathmandu valley. J. Nat. Hist. Mus., 3(1-4):83-93.

Dangol, D.R. 2001. Ecological studies and used of weeds of winter crop at Rampur, Chitwan. In Jha, P.K., S.R. Baral, S.B. Karmacharya, H.D. Lekhak, P. Lacoul and C.B. Baniya (eds) Environment and Agriculture: Biodiversity, Agriculture and Pollution in South Asia. Ecological Society, Kathmandu, Nepal, pp 430-434.

Dangol, D.R. and N.K. Chaudhary, 1994. Wheat-weed interactions at Rampur, Chitwan. In. F.P. Neupane (ed), IAAS Research Reports (1992-1993), Institure of Agriculture and Animal Science, Rampur, Chitwan, Nepal.

Dangol, D.R., 1987. Survey of weeds in wheat field at Birganj, Parsa, Nepal. J. Inst. Agric. Anim. Sci., 8:45-51.

Dangol, D.R., 1998. Agrestal weeds of Nepal: Identification, distribution, ecology and agricultural importance. Ph.D. Dissertation, University of Vienna, Austria.

Dangol, D.R., 2000-2001. A comparison of weedflora of wheat fields of Inner Terai and Terai of Nepal. J. Inst. Agric. Anim. Sci., 21-22:95-103.

Dangol, D.R., S.B. Gurung, I. Bhattarai and N.K. Chaudhary, 1988. Survey of weeds of major crops at Rampur and vicinity, in Chitwan, Nepal. J. Inst. Agric. Anim. Sci., 9:35-43.

Devkota, R.N., 1983. Weeds of wheat fields and their control (Nepali). Krishi, 20(5):20-24. 
Gupta, O.P., 1984. Scientific Weed Management. Today and Tomorrow's Printers and Publishers, New Delhi, India.

Gupta, O.P., S.R. Bajracharya and G.P. Shivakoti, 1977. A study of weed problems at Rampur, Chitwan, Nepal. J. Inst. Agric. Anim. Sci., 1:1-86

Hara, H. and L.H.J. Williams. 1979. An Enumeration of the Flowering Plants of Nepal. Volume 1. British Museum (Natural History), London.

Hara, H., A.O. Chater and L.H.J. Williams. 1979. An Enumeration of the Flowering Plants of Nepal. Volume 3. British Museum (Natural History), London.

Hara, H., W.T. Stearn and L.H.J. Williams. 1978. An Enumeration of the Flowering Plants of Nepal. Volume 2. British Museum (Natural History), London.

Holm, L.G., D.L. Pluncknett, J.V. Pancho, and J.P. Herberger, 1977. The World's Worst Weeds: Distribution and Biology. The University Press of Hawaii, USA.

Joshi, M. and R. Gretzmacher, 1999. Species composition and dominance of weeds in ricewheat cropping system of Kavre district, Nepal. Nepal Journal of Science and Technology, 1:19-26.

Malla, M.L. and J.D. Ranjit, 1980. Weed control experiment in wheat and barley. Wheat Report. HMG, Department of Agriculture, NWDP, Siddharthnagar, Bhairahawa, Nepal, pp 179-181.

Manandhar, N.P., 1978. A study of weeds on the river banks of Kathmandu Valley. Bull. Bot. Surv. India, 20(1-4):36-47.

Mazek, A., 1991. The Weed Vegetation of Nepal. Ph. D. Dissertation, University of Agriculture and Forestry, Institute of Botany, Vienna, Austria.

Nepal Wheat Production by Year. http://www.indexmundi.com/agriculture/?country= np\&commodity= wheat\&graph=production retrieved on July 6, 2013.

Numata, M., 1982. A methodology for the study of weed vegetation. In Holzner, W. and N. Numata (eds) Biology and Ecology of Weeds. Dr. W. Junk Publishers, The Hauge, pp 21-34. Rajbhandary, K.L. and S. Ojha, 1979. Notes on weeds associated with wheat crop. Nep. J. Agri., 14(13-14):49-51.

Ranjit, J.D., 2003. Response of wheat weeds to straw mulch in midhills. In Neupane, F.P., K.M. Bajracharya and D. Bhuju (eds), Proceedings of International Seminar on MountainsKathmandu, March 6-8, 2002. Royal Nepal Academy of Science and Technology, Kathmandu, Nepal, pp 372-377.

Ranjit, J.D. and A.N. Bhattarai, 1988. Crop weeds and their control in Nepal. Agricultural Research and Production Project. Winrock Internation/USAID Project No. 367-0149-3-50002, Kathmandu, Nepal.

Ranjit, J.D. and R. Suwanketnikom, 2003. Response of weeds and wheat yield to tillage and weed management. Kasetsart J. (Nat. Sci.), 37:389-400.

Ranjit, J.D., 1981/82. Weed control trial in wheat. Wheat Research Report. HMG, Department 
of Agriculture, NWDP, Siddharthnagar, Bhairahawa, Nepal, pp 141-248.

Ranjit, J.D., 1983a. Common weeds of wheat crop and their control (Nepali). Krishi, 20(4):17-19. Ranjit, J.D., 1983b. Weed control trial on wheat. Wheat Report, HMG, Department of Agriculture, NWDP, Siddharthnagar, Bhairahawa, Nepal, pp 365-577.

Ranjit, J.D., 1999. Study on the effect of tillage and weed management in wheat. Nepal Agriculture Research Journal, 3:28-35.

Ranjit, J.D., 2009. Impact of mulching on wheat yield and weed floras in the mid-hills of Nepal. Nepal Agriculture Research Journal, 9:21-26.

Ranjit, J.D., R.R. Bellinder, P. Hobbs, N.K. Rajbhandari and P. Kataki, 2006. Mapping Phalaris minor under the rice-wheat cropping system in different agro-ecological regions of Nepal. Nepal Agriculture Research Journal, 7:54-63.

Regmi, P.P., P.L. Karna and L.S. Karki, 1986. Weed problems of wheat crop in Nepal (typed manuscript).

Sapkota, N., B.R. Dongol and D.R. Bhuju, 2010. Weed species composition and growth in wheat field of mountain ecosystem Khokana, Lalitpur, Nepal. Botanica Orientalis, 7:85-91.

Shah, P., K.R. Dahal, S.K. Shah and D.R. Dangol, 2011a. Effect of tillage, mulch and time of nitrogen application on yield of wheat (Triticum aestivum L.). Agriculture Development Journal, 8:9-19.

Shah, P., K.R. Dahal, S.K. Shah and D.R. Dangol, 2011b. Weed proliferation as affected by long-term tillage, mulch and time of nitrogen application in wheat (Triticum aestivum L.). International Research Journal of Applied and Basic Sciences, 2(2):65-72.

Sharma, L.N., R.P. Chaudhary and V.N.P. Gupta, 2010. Weed diversity in the trans-himalayan wheat fields of Upper Manang, Nepal. In: Jha, P.K., S.B. Karmacharya, M.K. Balla, M.K. Chettri and B.B. Shrestha (eds) Sustainable Use of Biological Resources in Nepal, Ecological Society (ECOS), Kathmandu, pp 72-78.

Shivakoti, G.P., O.P. Gupta and S.R. Bajracharya, 1977. Response of wheat, rape and potato to weeding. J. Inst. Agric. Anim. Sci., 1 (1):103-109.

Sthapit, B.R., 1986. Herbicidal control of various hill weeds in the wheat field. Nepalese J. Agric., 17:125-127.

Thapa, C.B. and R.S. Kayastha, 1996. Weed flora of wheat field in Pokhara Valley. Prithwi: A Research Publication of P.N.C., 1(1):1-8.

Thapa, C.B., 2003. Survey of weed flora of wheat field in Pokhara, Nepal. Botanica Orientalis (Annual Issue), pp 144-145. 


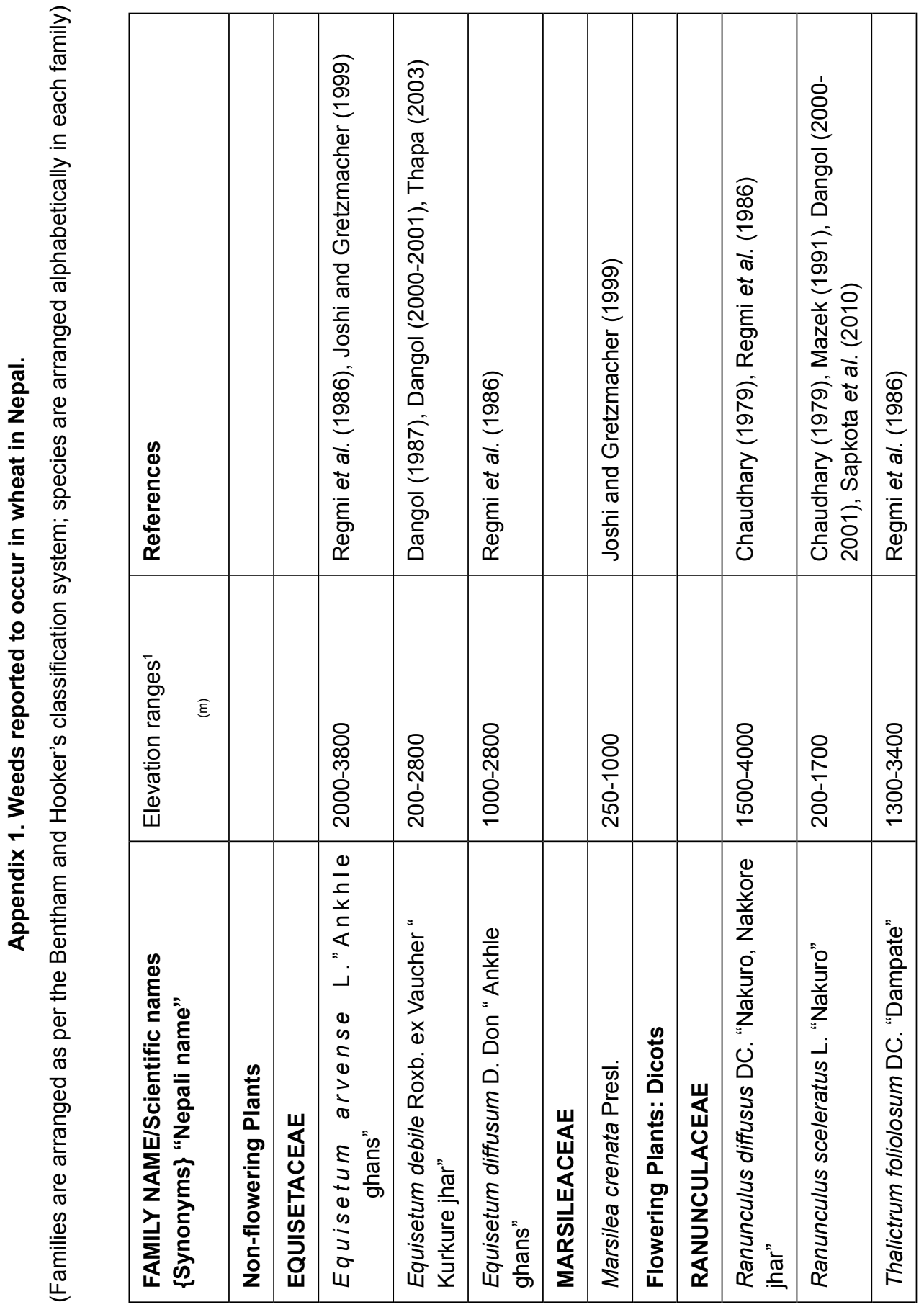




\begin{tabular}{|c|c|c|c|c|c|c|c|c|c|c|c|}
\hline & 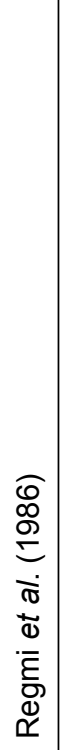 & & 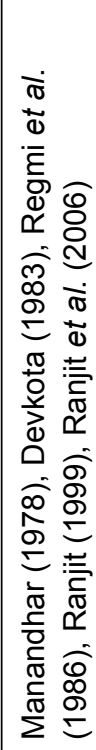 & 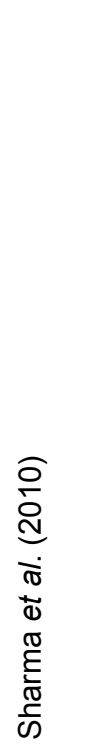 & & 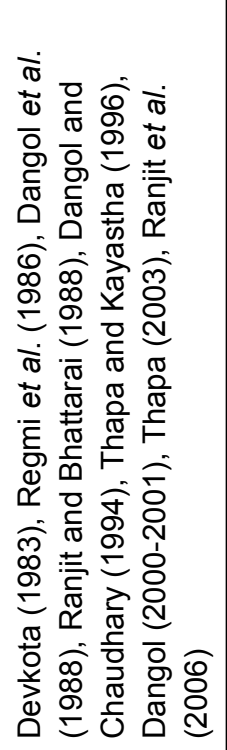 & & 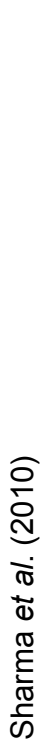 & & 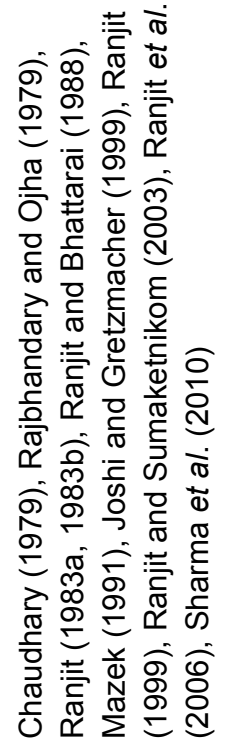 & 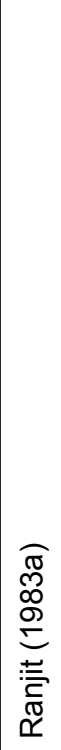 \\
\hline & 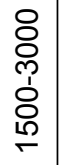 & & 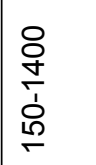 & $\begin{array}{l}\text { م⿱ } \\
\text { Oे } \\
\text { d } \\
\text { लூ }\end{array}$ & & 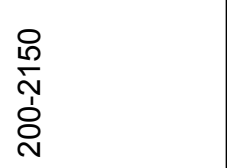 & & 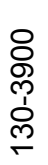 & & $\begin{array}{l}\text { ○ } \\
\text { గீ } \\
\text { †े } \\
\text { ᄋ }\end{array}$ & $\begin{array}{l}8 \\
8 \\
0 \\
+ \\
0 \\
8 \\
10\end{array}$ \\
\hline 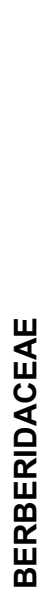 & 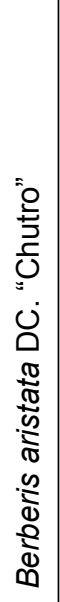 & 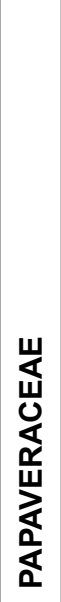 & 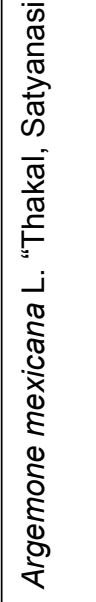 & 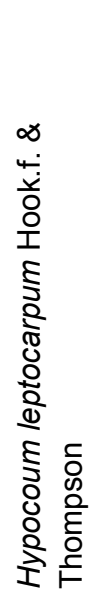 & 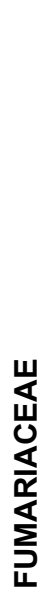 & 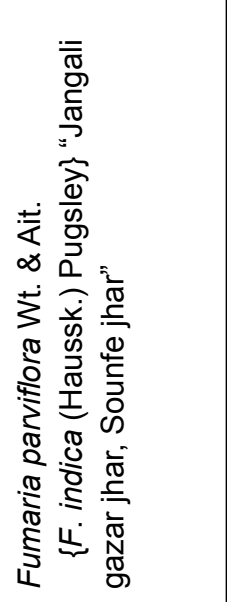 & 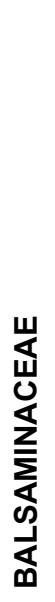 & 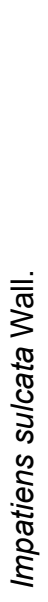 & 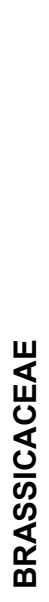 & 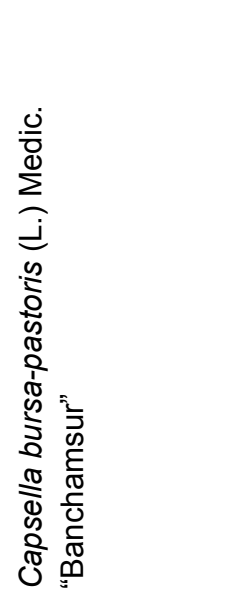 & 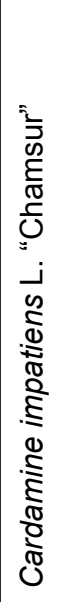 \\
\hline
\end{tabular}




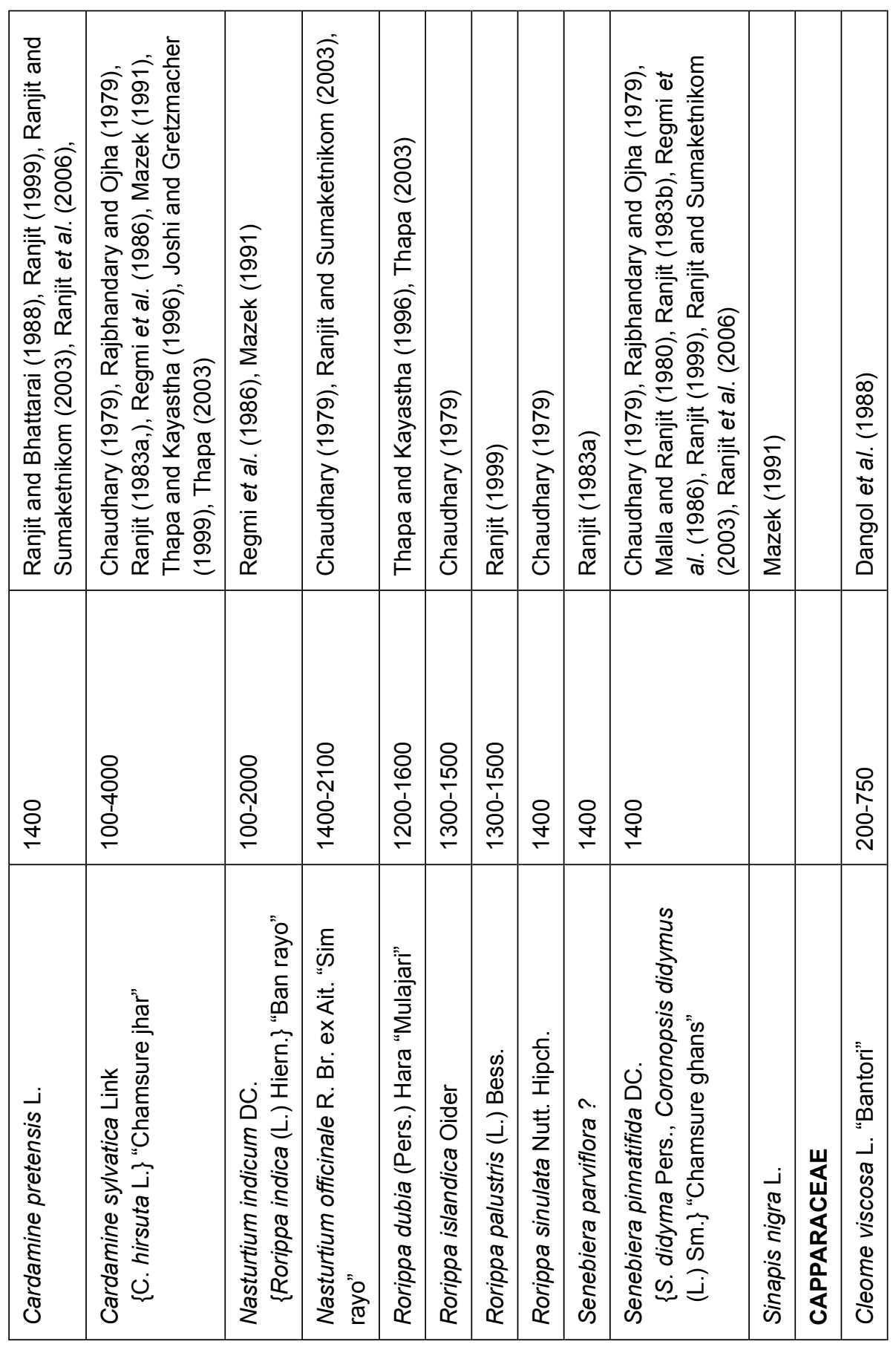




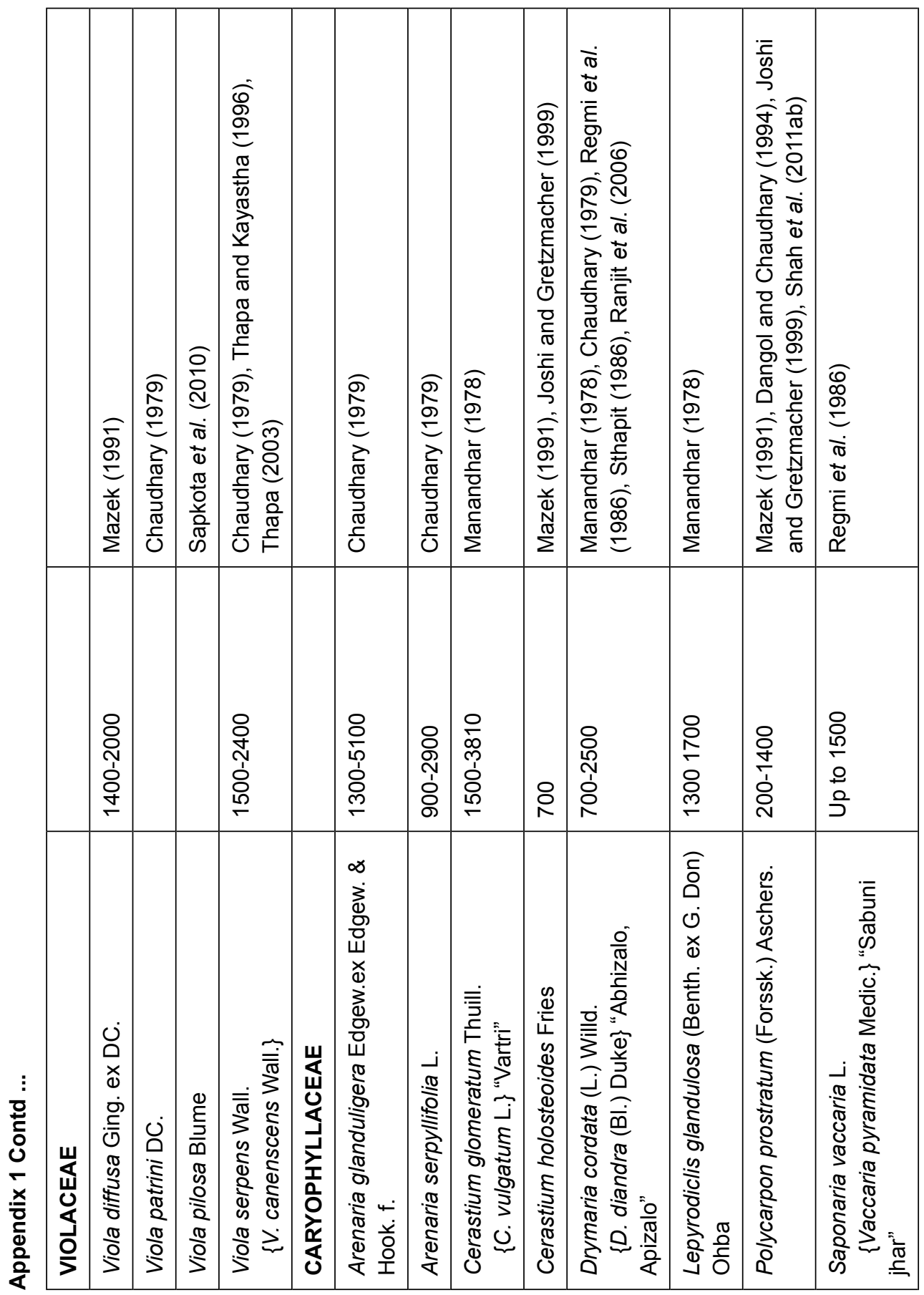




\begin{tabular}{|c|c|c|c|c|c|c|c|c|c|c|c|c|}
\hline 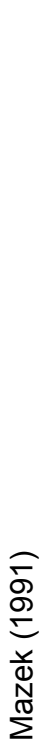 & 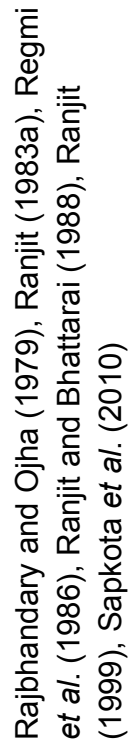 & 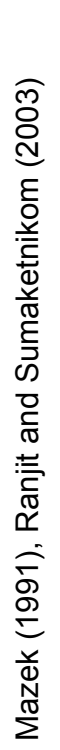 & 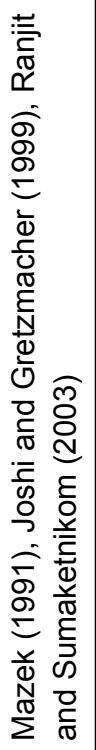 & 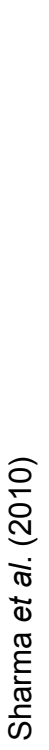 & 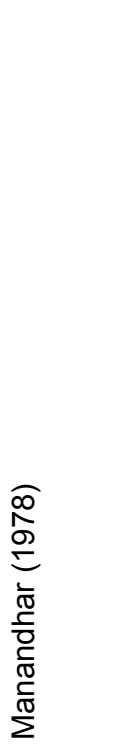 & 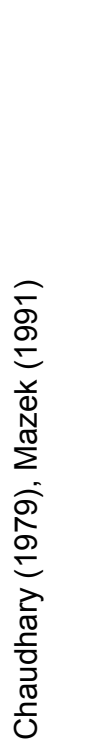 & 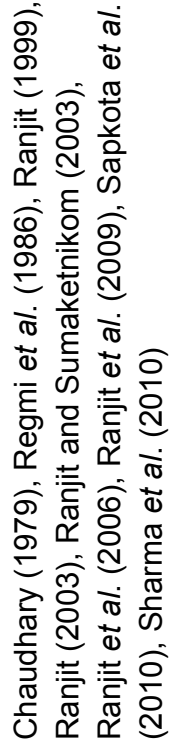 & 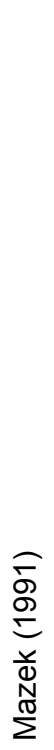 & 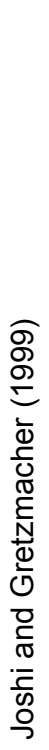 & & 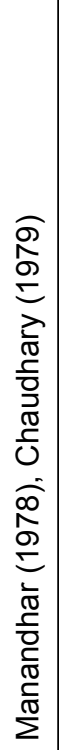 & \\
\hline 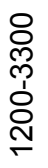 & $\frac{8}{8}$ & 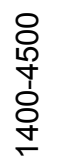 & $\stackrel{\text { \& }}{\stackrel{+}{+}}$ & 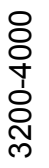 & 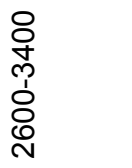 & 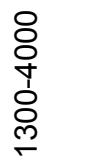 & 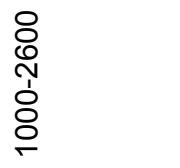 & 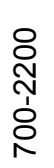 & 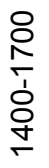 & & 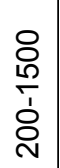 & \\
\hline 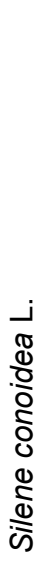 & 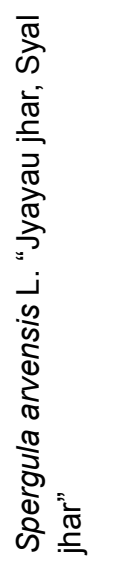 & 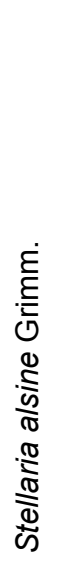 & 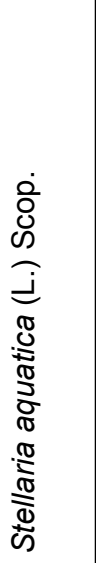 & 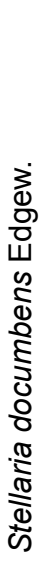 & 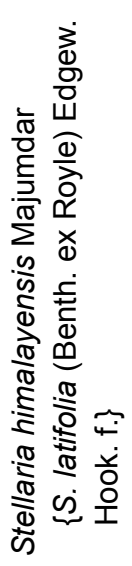 & 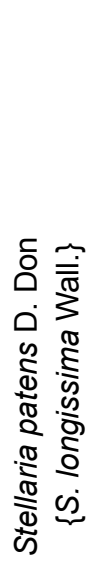 & 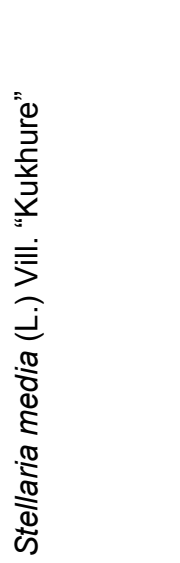 & 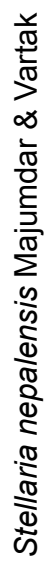 & 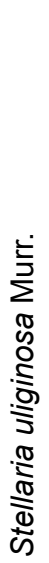 & 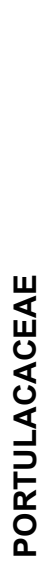 & 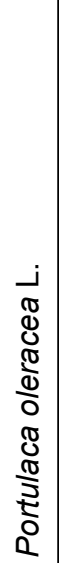 & 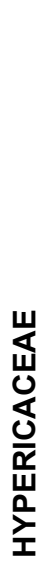 \\
\hline
\end{tabular}




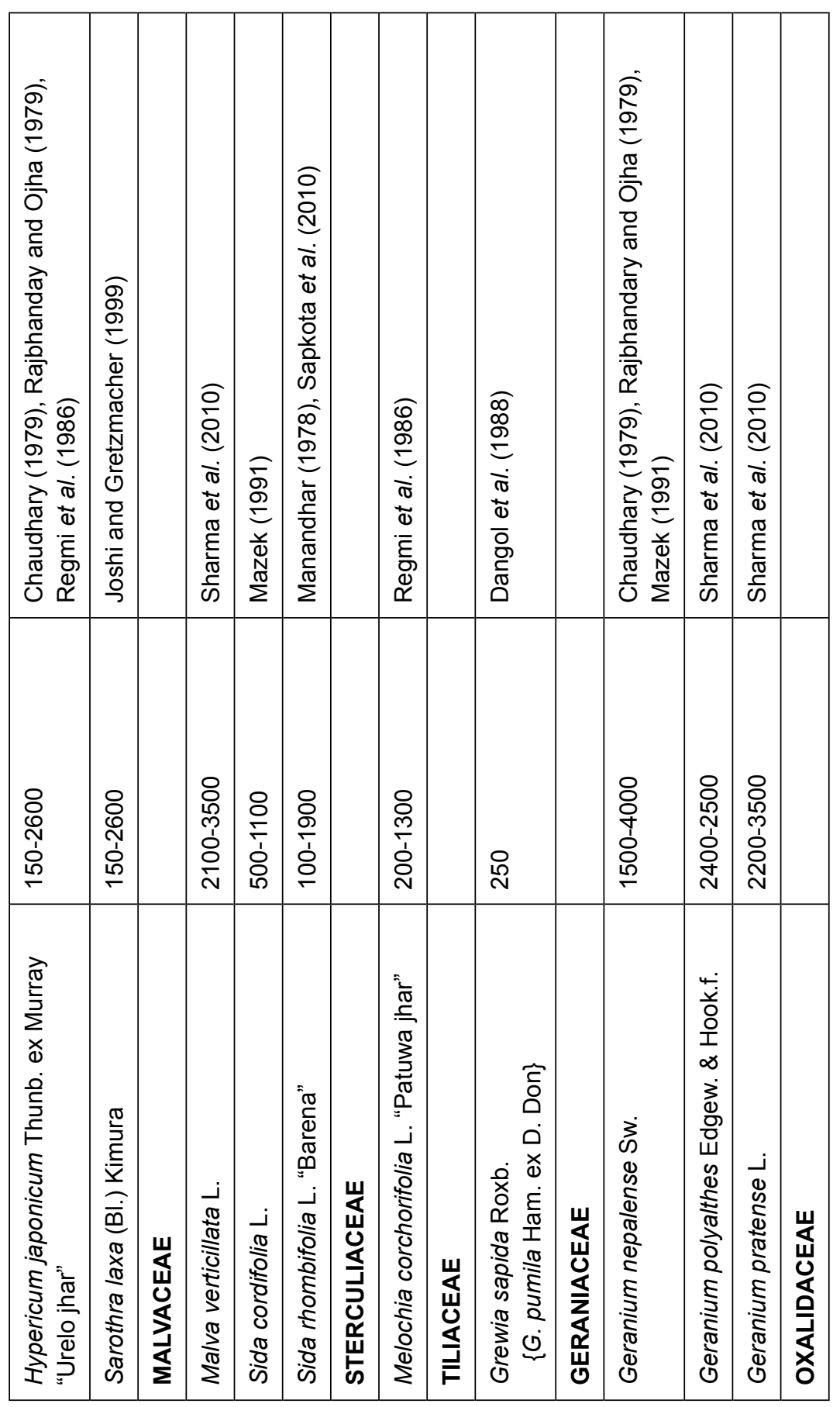




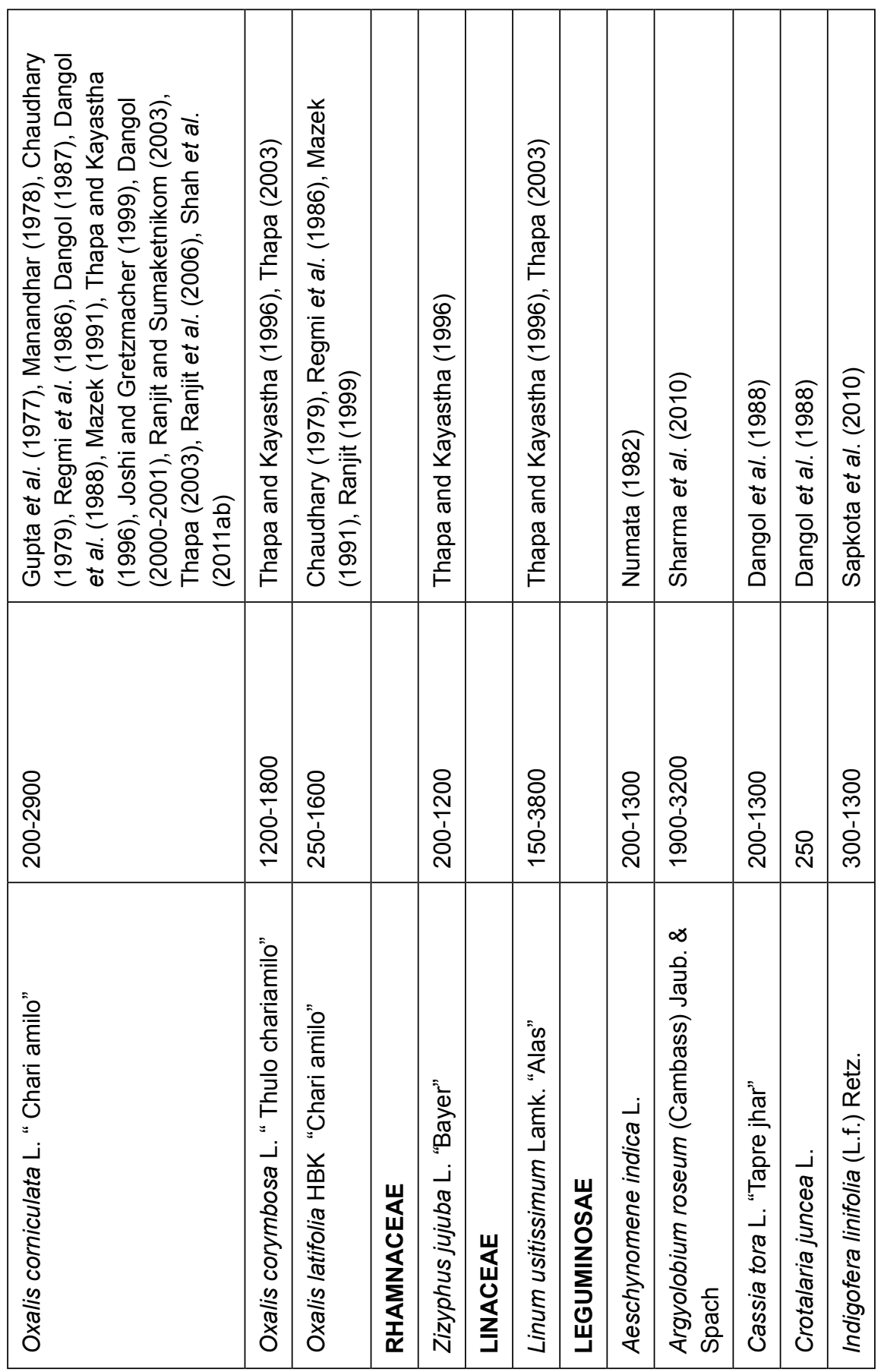




\begin{tabular}{|c|c|c|c|c|c|c|c|c|c|}
\hline 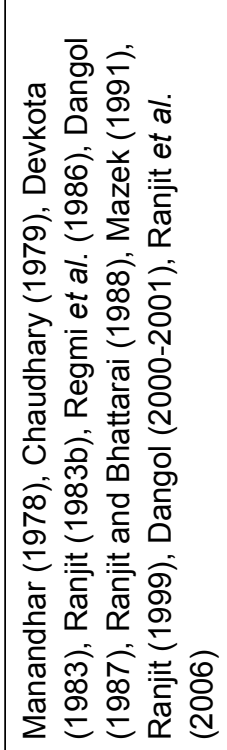 & 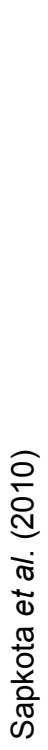 & 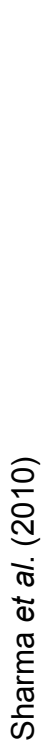 & 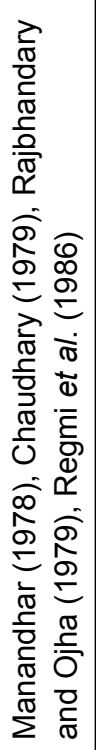 & 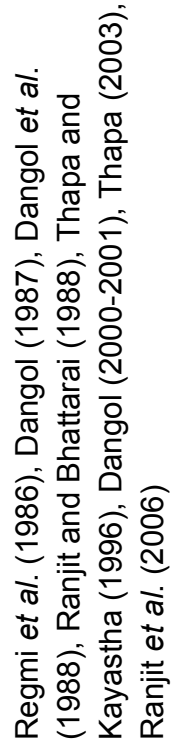 & 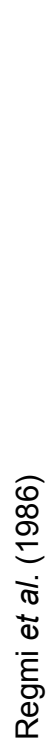 & 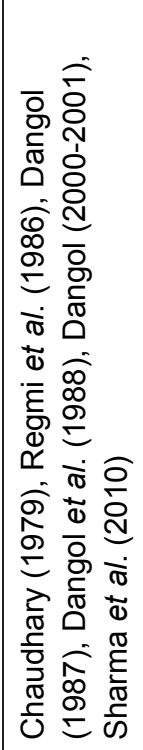 & 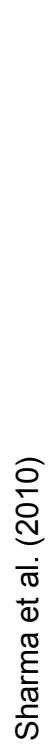 & 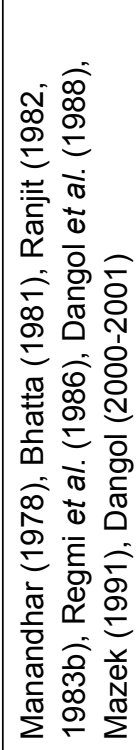 & 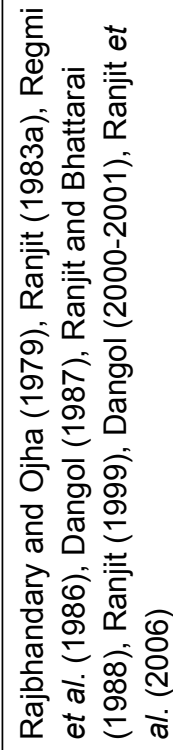 \\
\hline 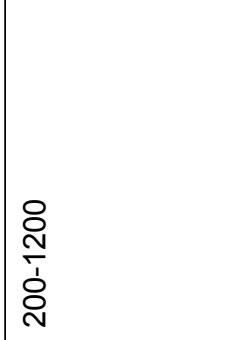 & $\begin{array}{l}8 \\
\frac{1}{1} \\
\frac{1}{2}\end{array}$ & 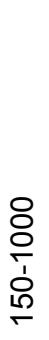 & $\begin{array}{l}\text { ठ } \\
\text { m } \\
\text { ᄋ } \\
\text { 임 }\end{array}$ & $\begin{array}{l}\text { ᄋ } \\
\text { +े } \\
\text { ᄋ̊ }\end{array}$ & 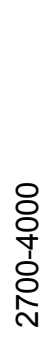 & $\begin{array}{l}\text { 오 } \\
\text { ச } \\
\text { ○े } \\
\text { ᄋ }\end{array}$ & ষ্ণ & $\frac{\text { 옹 }}{\text { 어 }}$ & $\begin{array}{l}\text { ᄋ } \\
\text { ల్ } \\
\text { ’ } \\
\text { ㅇ }\end{array}$ \\
\hline 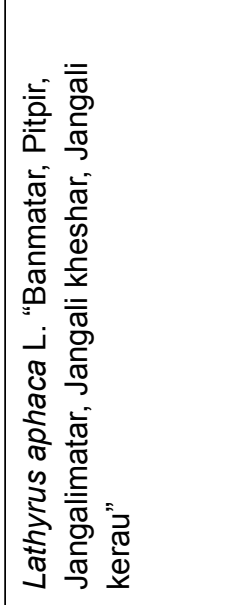 & 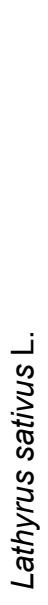 & 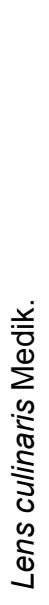 & 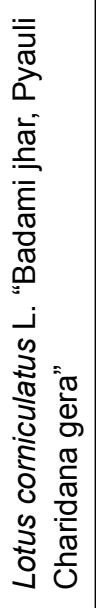 & 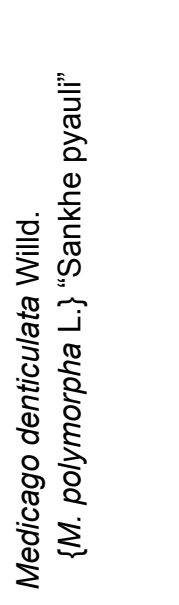 & 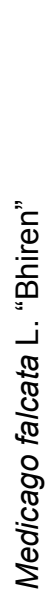 & 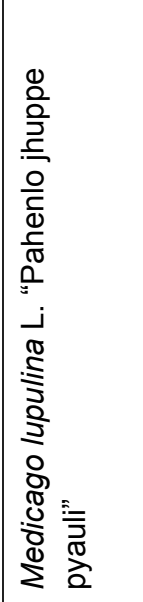 & 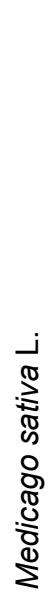 & 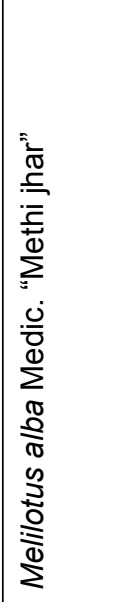 & 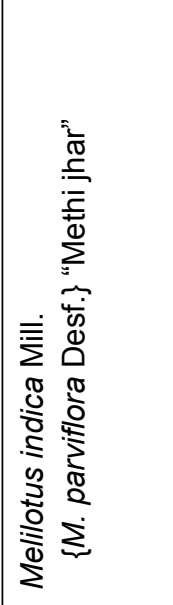 \\
\hline
\end{tabular}




\begin{tabular}{|c|c|c|c|c|c|c|c|c|}
\hline 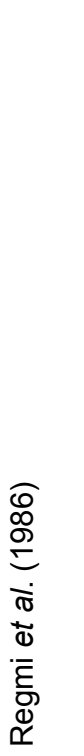 & 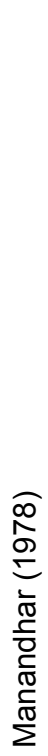 & 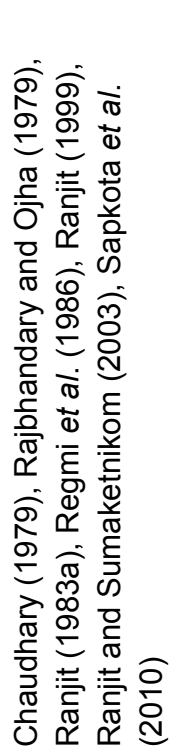 & 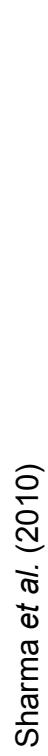 & 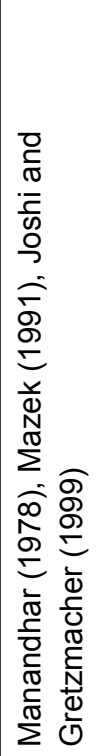 & 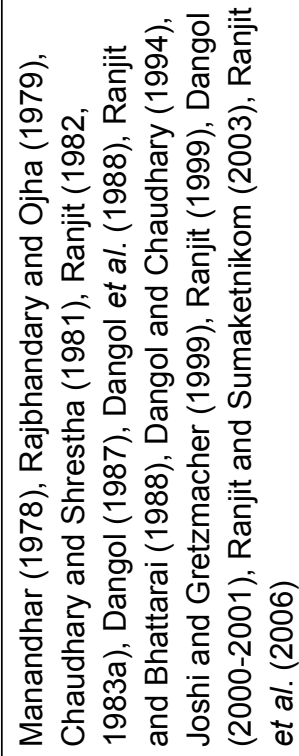 & 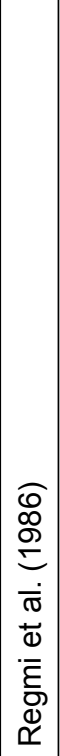 & 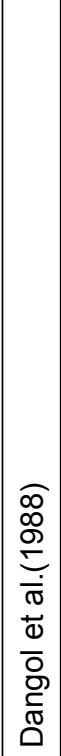 & 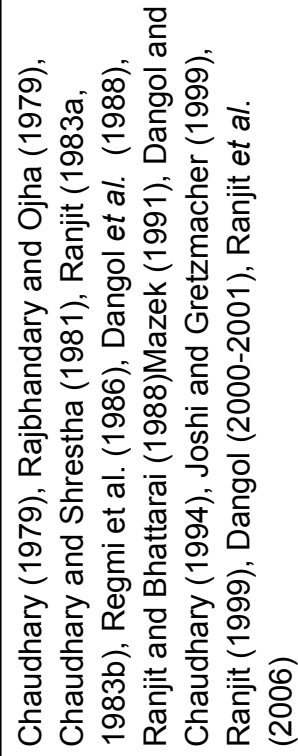 \\
\hline 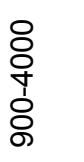 & 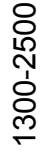 & 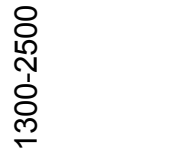 & $\begin{array}{l}\text { ᄋ } \\
\frac{8}{1} \\
\text { ᄋ } \\
\frac{\infty}{-}\end{array}$ & $\begin{array}{l}\text { ○ } \\
\text { ச } \\
\text { ণ } \\
\text { ঠ }\end{array}$ & $\begin{array}{l}8 \\
\text { N } \\
\text { ò } \\
\text { ㅇ }\end{array}$ & & 유 & 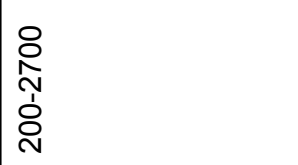 \\
\hline 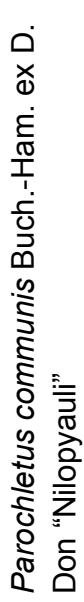 & 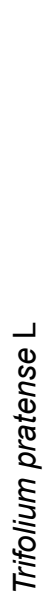 & 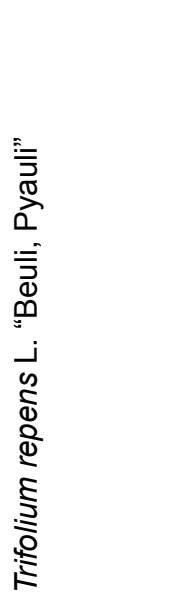 & 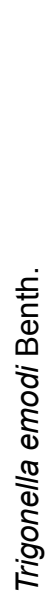 & 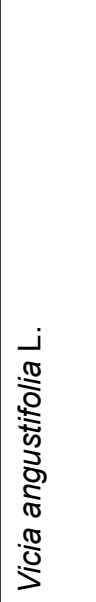 & 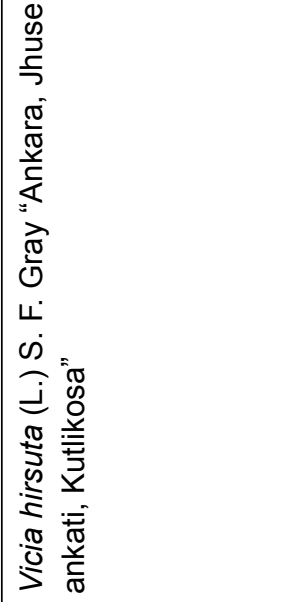 & 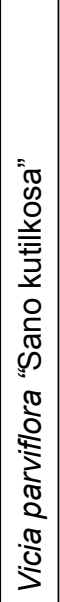 & 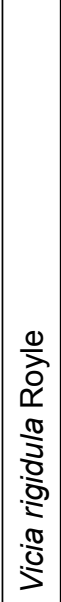 & 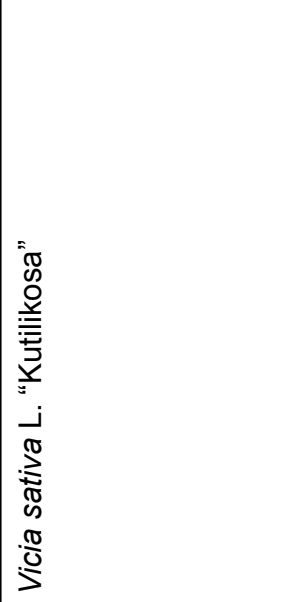 \\
\hline
\end{tabular}




\begin{tabular}{|c|c|c|c|c|c|c|c|c|c|c|c|c|}
\hline 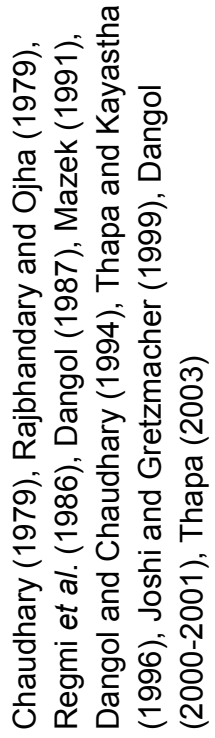 & & 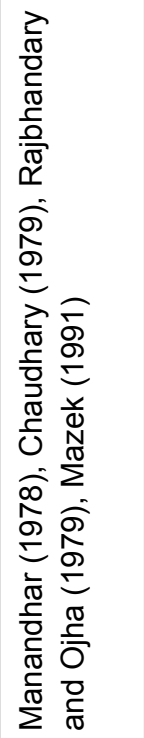 & 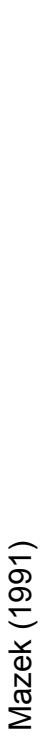 & 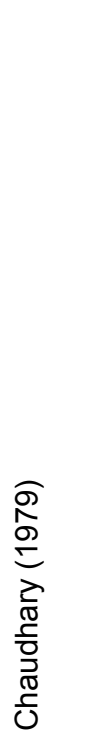 & 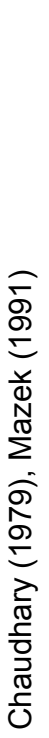 & 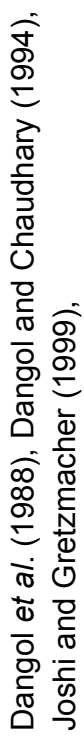 & & 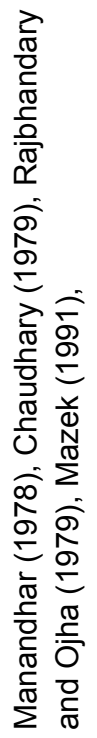 & 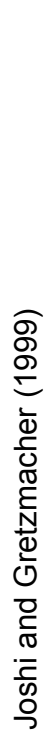 & & 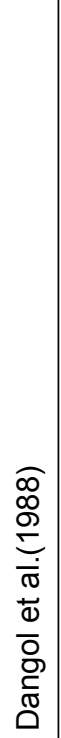 & 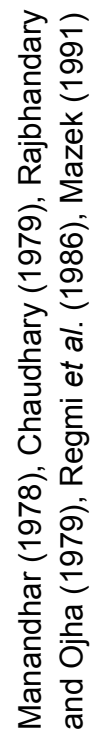 \\
\hline 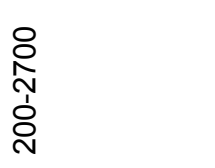 & & $\begin{array}{l}\text { 오 } \\
\text { స్} \\
\text { ঠे } \\
\text { ᄋ }\end{array}$ & 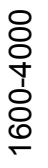 & 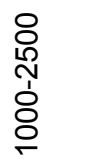 & 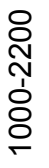 & $\begin{array}{l}\text { 옹 } \\
\text { 옹 }\end{array}$ & & $\begin{array}{l}\text { \& } \\
\text { N్} \\
\text { ஸे } \\
\text { م }\end{array}$ & $\begin{array}{l}\text { 尺े } \\
\text { } \\
\text { ஸे } \\
\text { مै }\end{array}$ & & 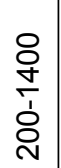 & 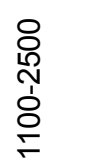 \\
\hline 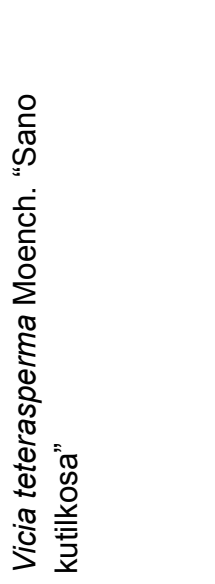 & 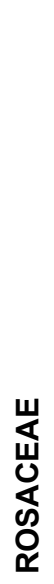 & 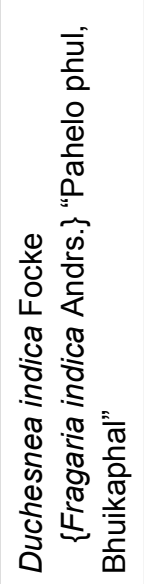 & 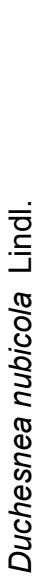 & 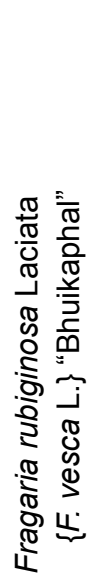 & 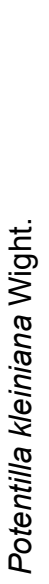 & 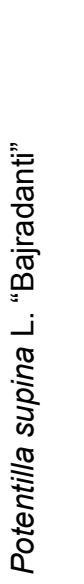 & 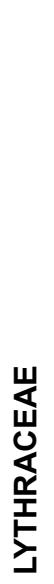 & 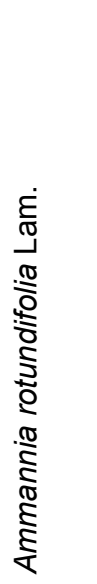 & 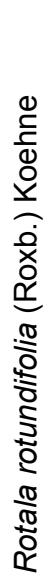 & 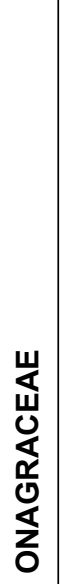 & 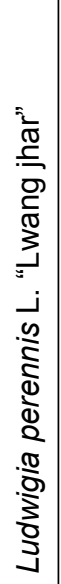 & 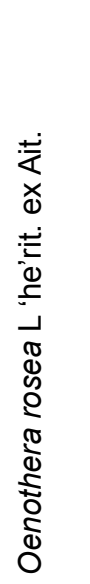 \\
\hline
\end{tabular}




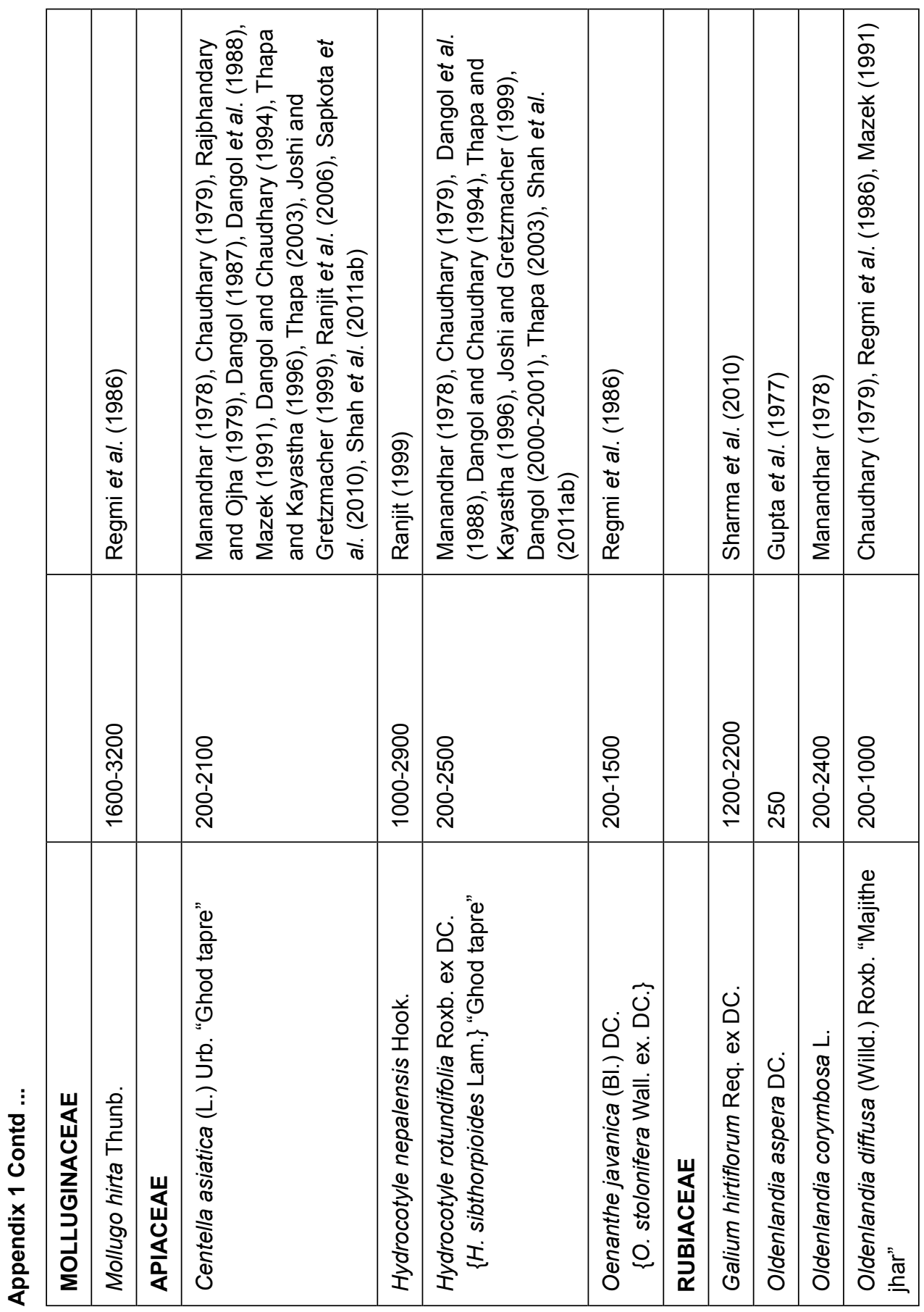




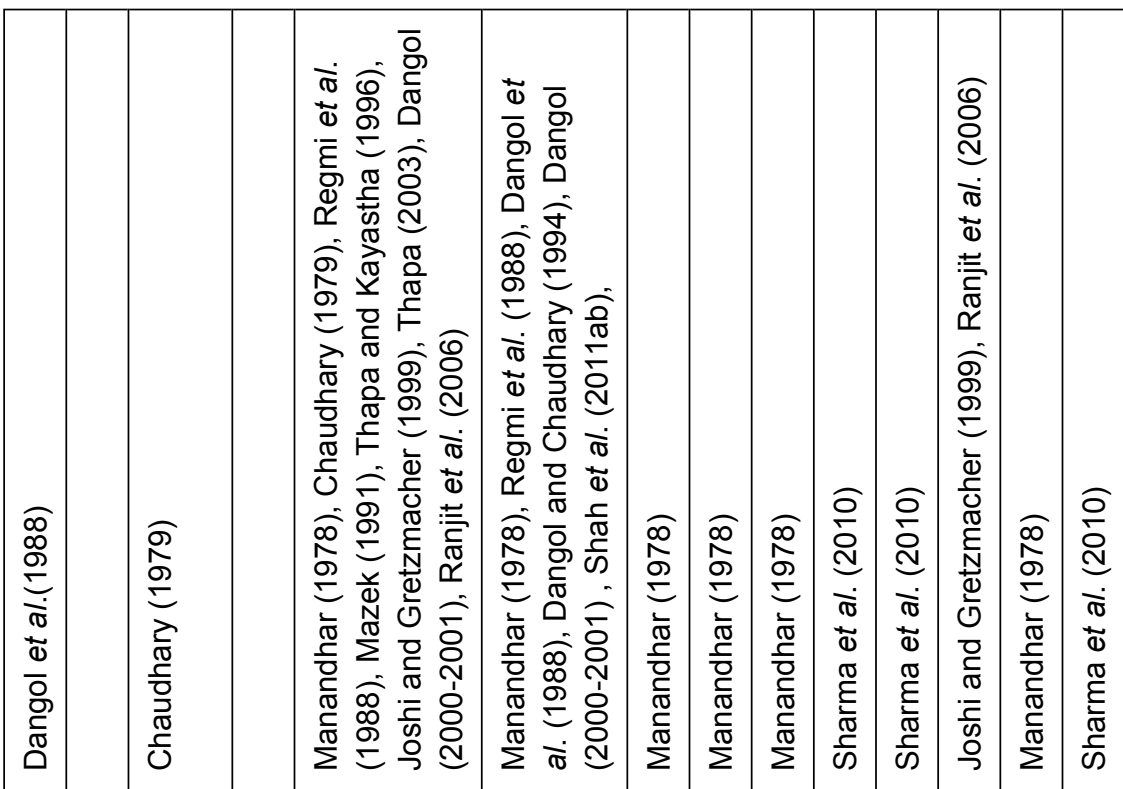

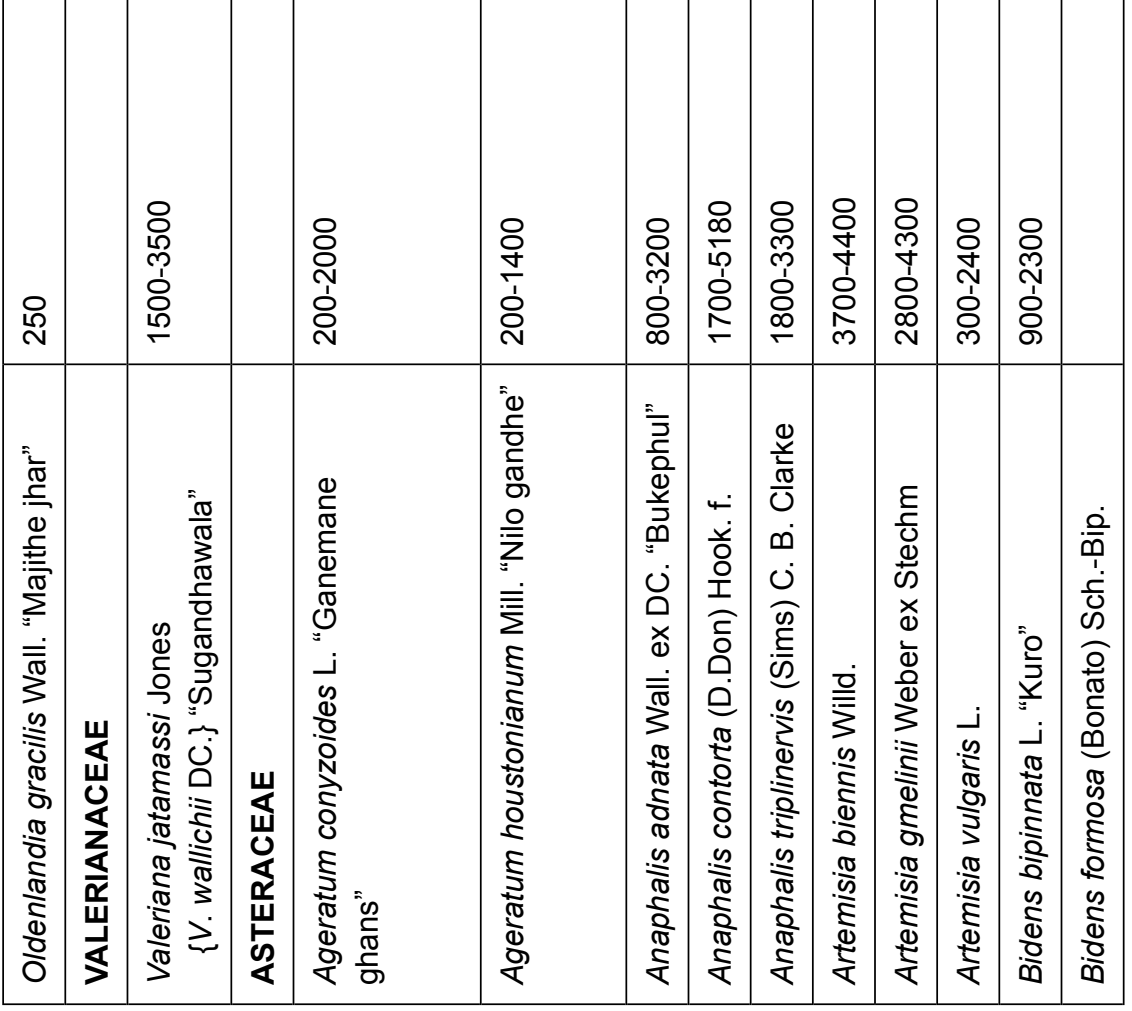




\begin{tabular}{|c|c|c|c|c|c|c|c|c|c|}
\hline 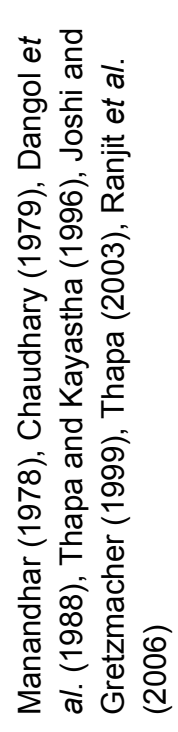 & 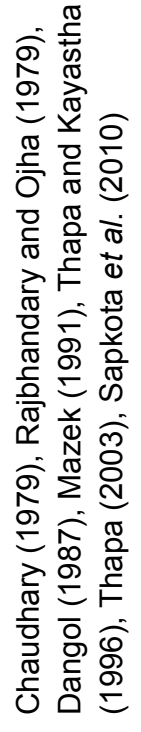 & 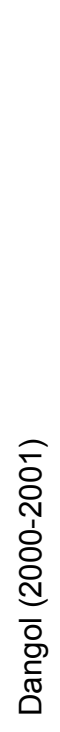 & 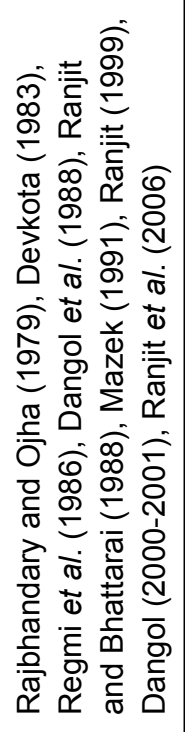 & 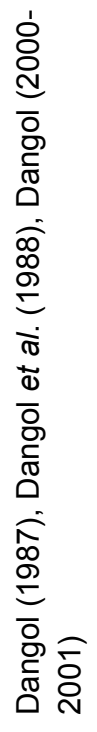 & 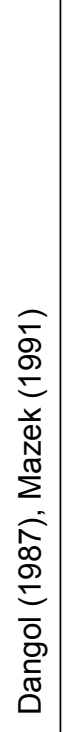 & 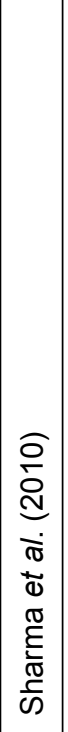 & 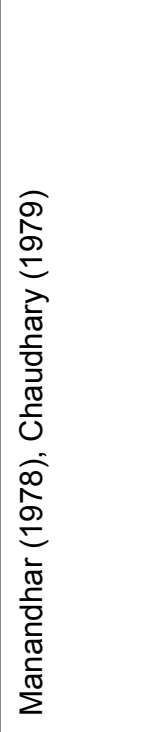 & 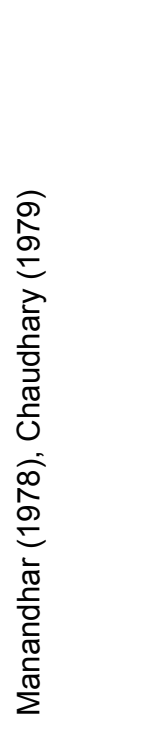 & 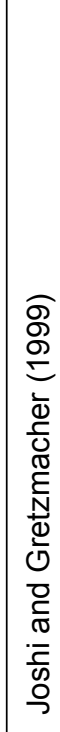 \\
\hline 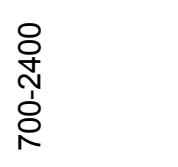 & 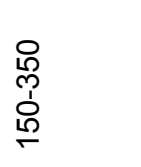 & 吕 & $\frac{8}{\frac{8}{1}}$ & 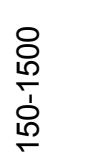 & $\begin{array}{l}\stackrel{8}{0} \\
\frac{0}{\grave{d}} \\
\stackrel{N}{ }\end{array}$ & 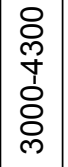 & $\begin{array}{l}0 \\
\text { h } \\
\text { م } \\
\text { h } \\
\text { م. }\end{array}$ & 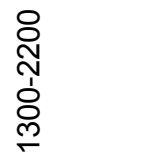 & 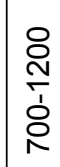 \\
\hline 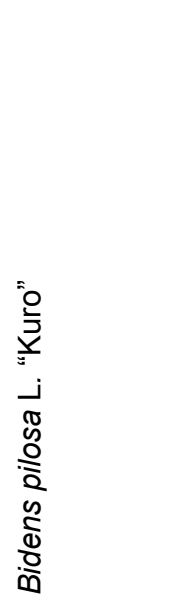 & 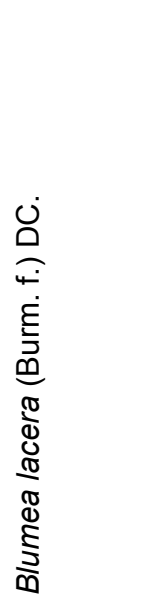 & 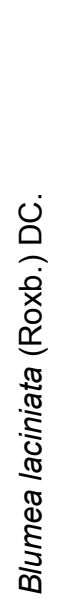 & 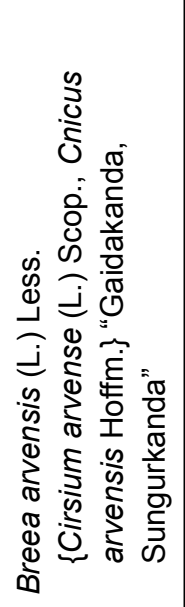 & 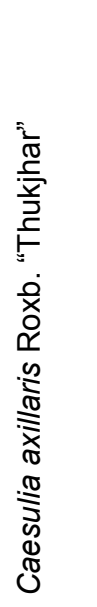 & 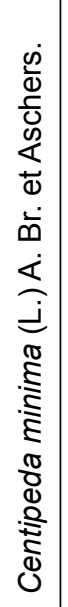 & 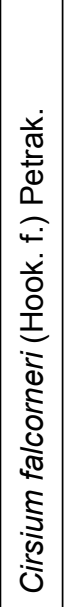 & 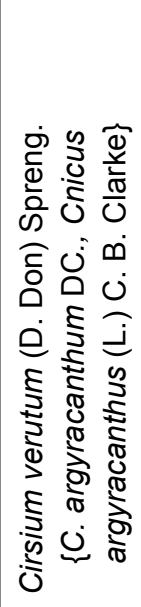 & 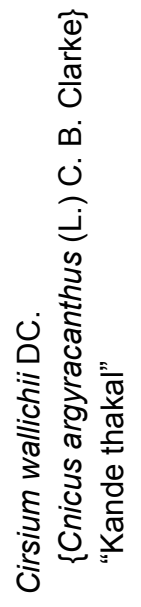 & 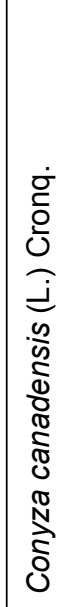 \\
\hline
\end{tabular}




\begin{tabular}{|c|c|c|c|c|c|c|c|c|c|c|}
\hline 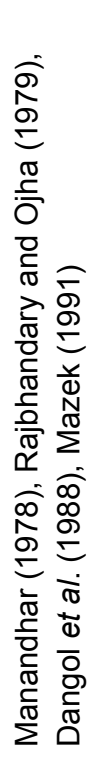 & 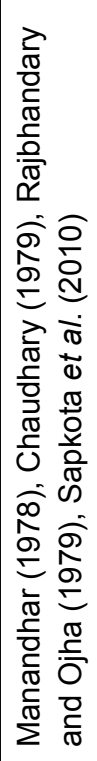 & 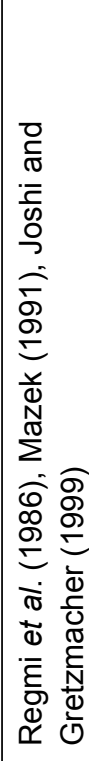 & 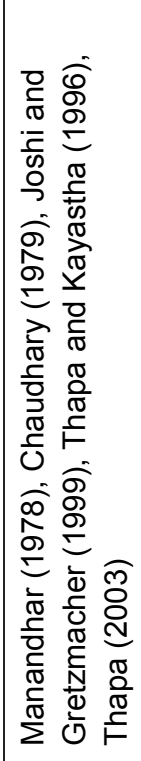 & 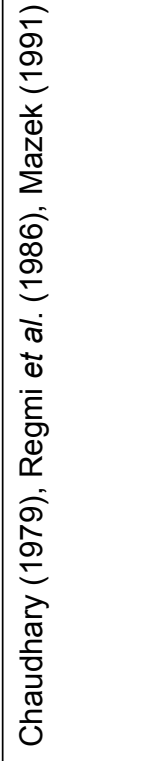 & 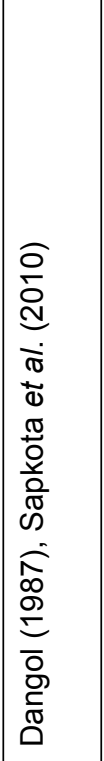 & 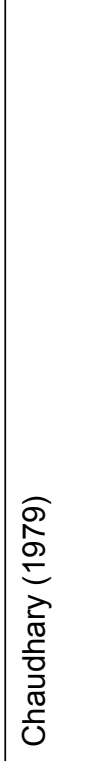 & 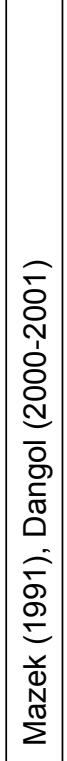 & 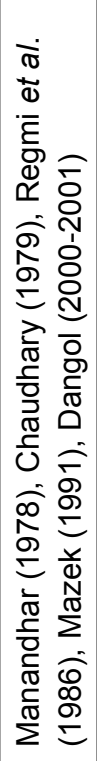 & 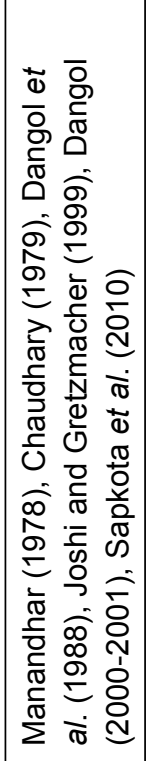 & 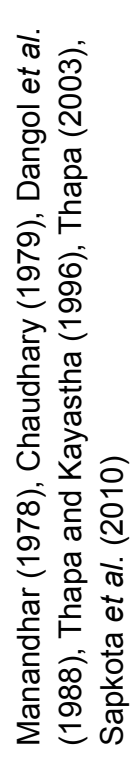 \\
\hline $\begin{array}{l}\text { రి } \\
\text { ஸे } \\
\text { ᄋి }\end{array}$ & $\begin{array}{l}8 \\
\text { ণิ } \\
\text { ठ̀े }\end{array}$ & 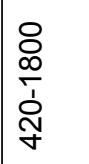 & 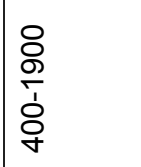 & 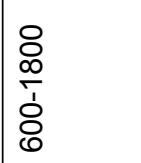 & $\begin{array}{l}\text { Oे } \\
\text { N̦ } \\
\text { రి } \\
0\end{array}$ & 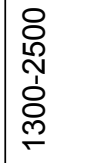 & $\begin{array}{l}8 \\
0 \\
N \\
1 \\
b \\
N\end{array}$ & 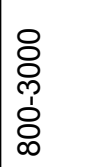 & 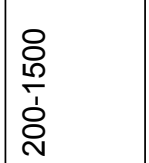 & 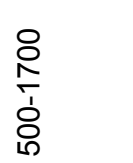 \\
\hline 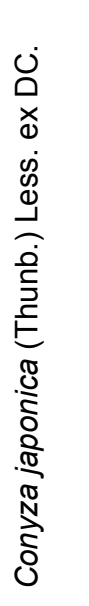 & 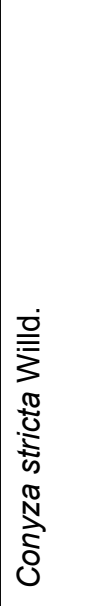 & 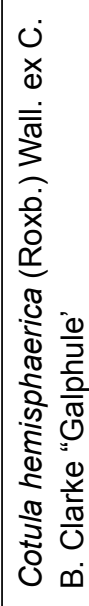 & 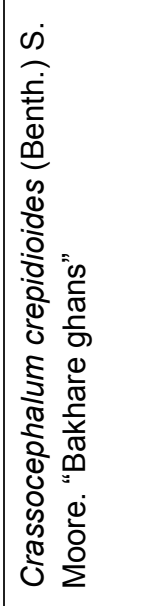 & 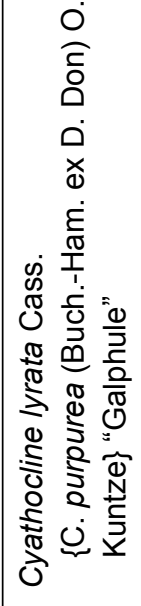 & 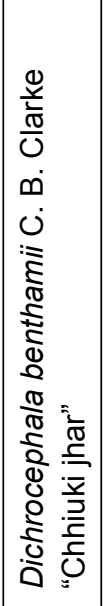 & 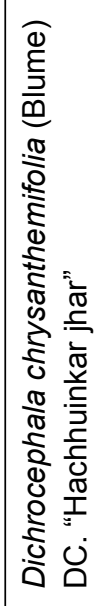 & 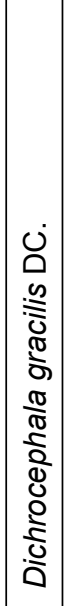 & 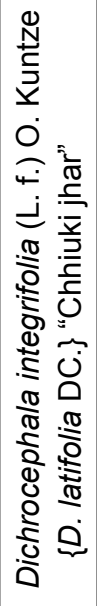 & 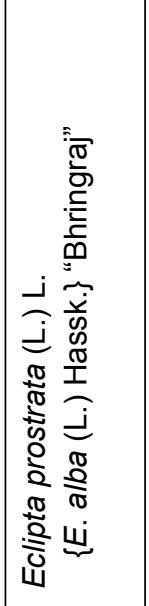 & 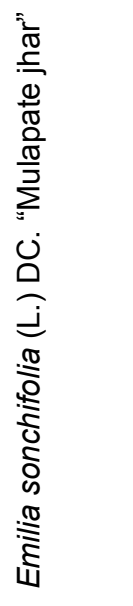 \\
\hline
\end{tabular}




\begin{tabular}{|c|c|c|c|c|c|c|c|c|}
\hline 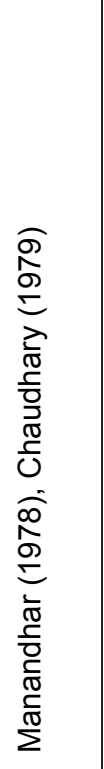 & 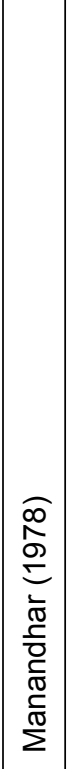 & 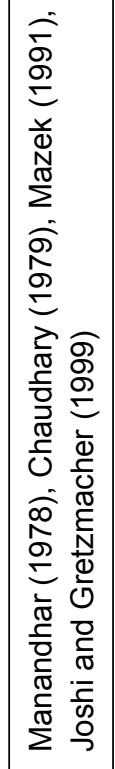 & 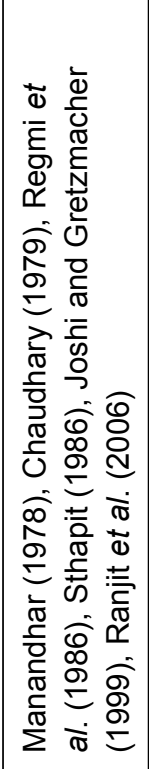 & 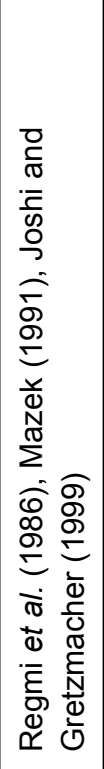 & 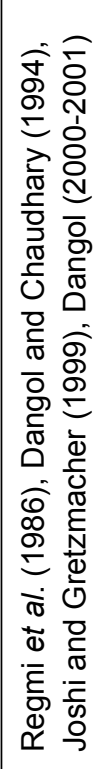 & 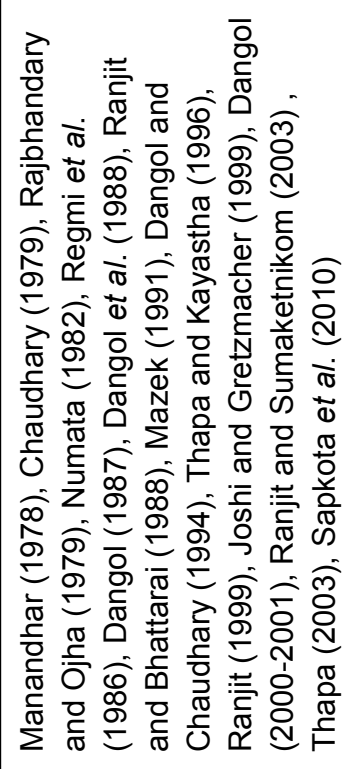 & 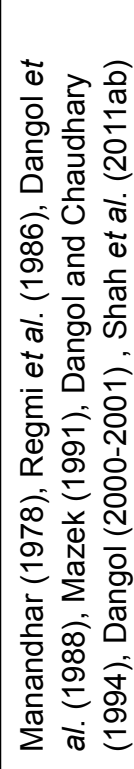 & 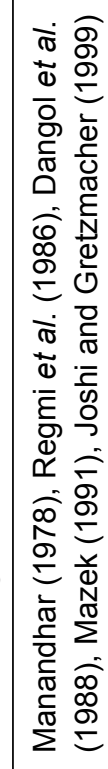 \\
\hline 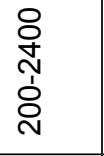 & 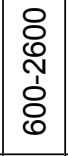 & $\begin{array}{l}\text { \& } \\
\stackrel{+}{*} \\
\text { d } \\
\infty \\
\infty\end{array}$ & 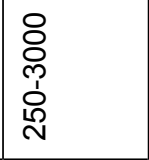 & 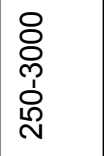 & $\frac{8}{\text { ò }}$ & 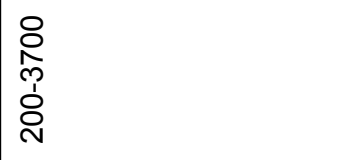 & $\frac{\text { ơ }}{\frac{+}{\grave{d}}}$ & 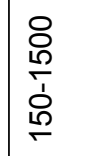 \\
\hline 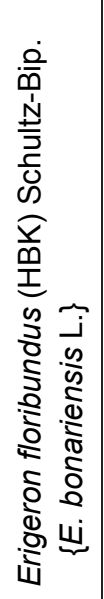 & 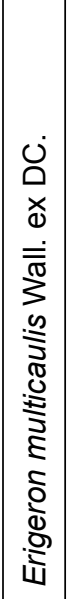 & 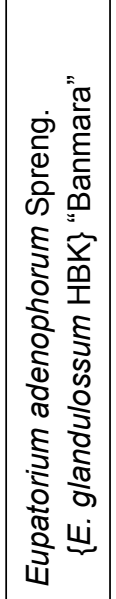 & 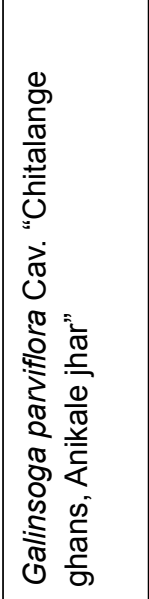 & 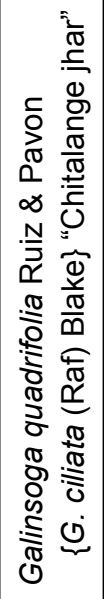 & 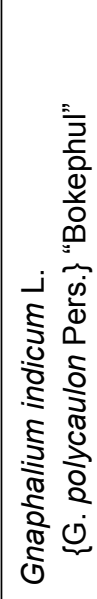 & 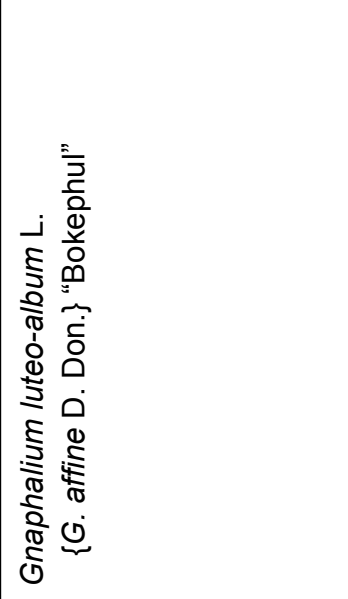 & 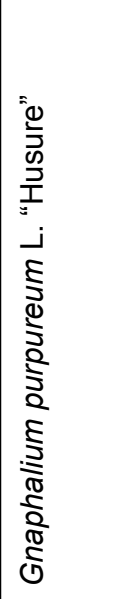 & 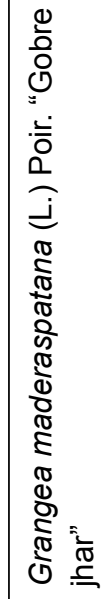 \\
\hline
\end{tabular}




\begin{tabular}{|c|c|c|c|c|c|c|c|c|c|c|c|c|c|c|}
\hline 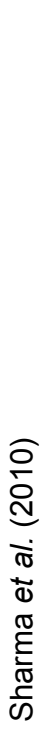 & 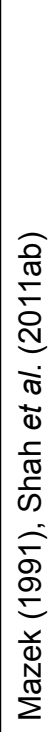 & 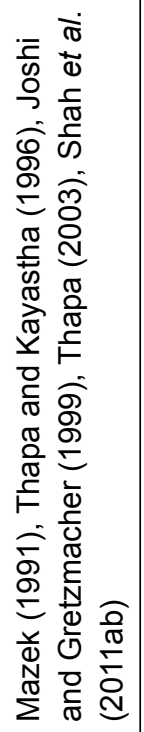 & 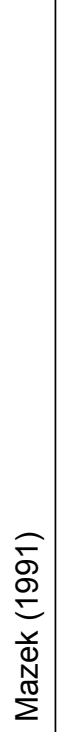 & 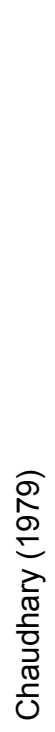 & 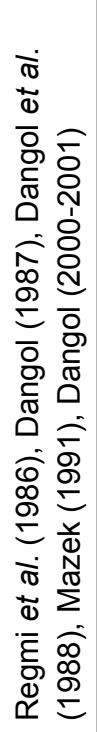 & 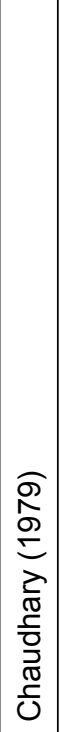 & 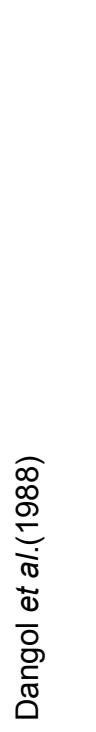 & 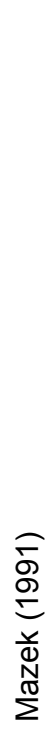 & 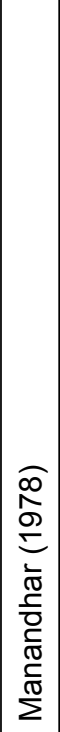 & 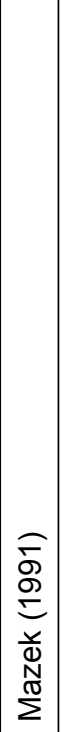 & 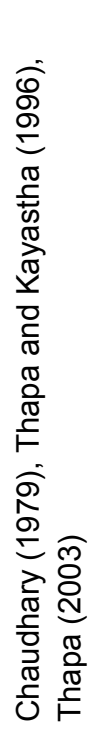 & 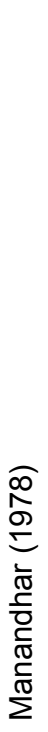 & 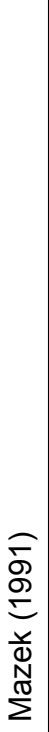 & 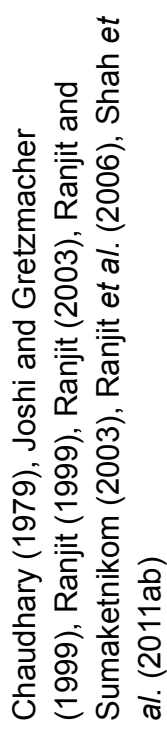 \\
\hline $\begin{array}{l}\text { ㅇ } \\
\text { О } \\
\text { ஸे } \\
\stackrel{2}{\sim}\end{array}$ & ষ্ণ & 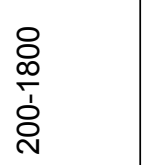 & $\begin{array}{l}8 \\
\text { ñ } \\
\delta \\
\varnothing \\
\circ\end{array}$ & 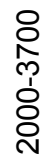 & $\begin{array}{l}\stackrel{8}{\circ} \\
\text { ণิ } \\
\text { ঠे }\end{array}$ & $\begin{array}{l}8 \\
\\
+\end{array}$ & 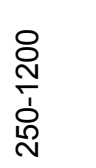 & 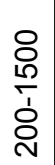 & $\begin{array}{l}0 \\
\infty \\
\infty \\
0 \\
\delta \\
\infty \\
\infty\end{array}$ & 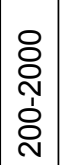 & 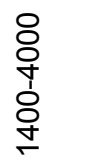 & $\underset{8}{\stackrel{8}{+}}$ & 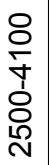 & 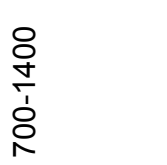 \\
\hline 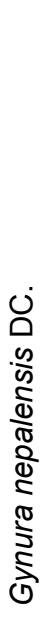 & 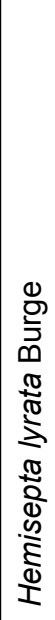 & 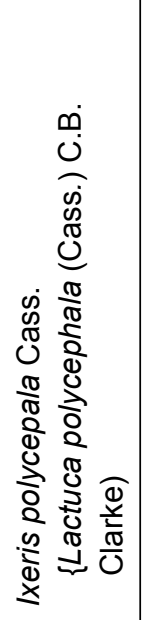 & 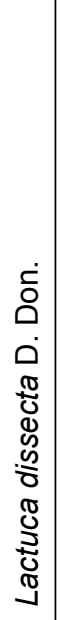 & 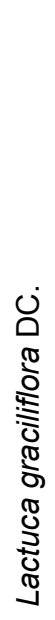 & 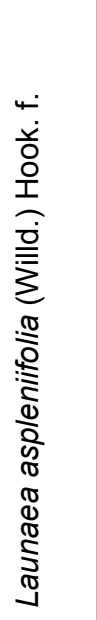 & 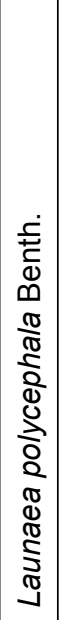 & 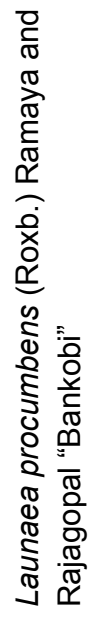 & 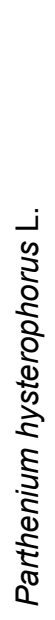 & 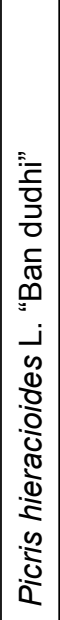 & 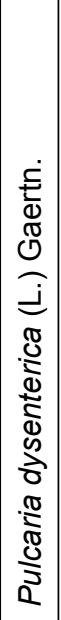 & 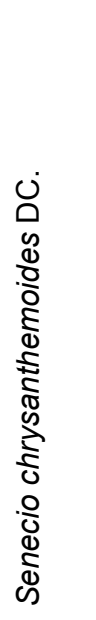 & 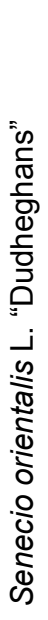 & 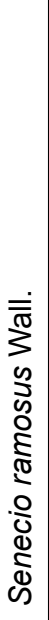 & 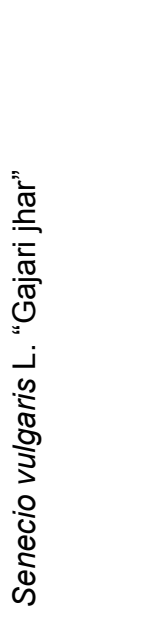 \\
\hline
\end{tabular}




\begin{tabular}{|c|c|c|c|c|c|c|c|c|c|c|}
\hline 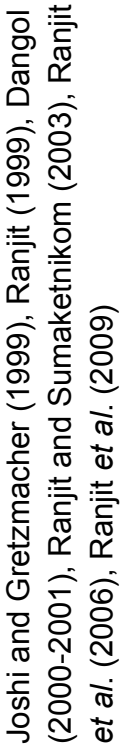 & 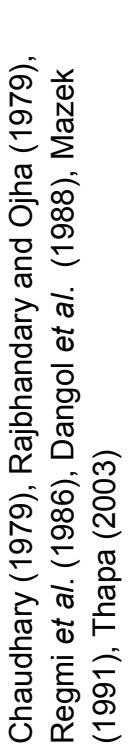 & 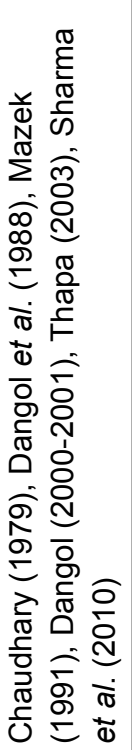 & 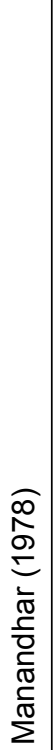 & 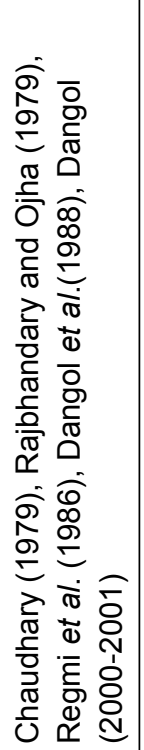 & 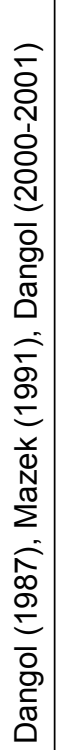 & 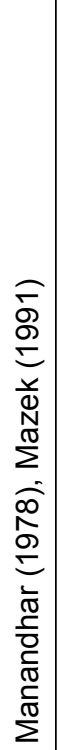 & 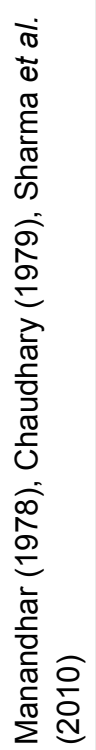 & 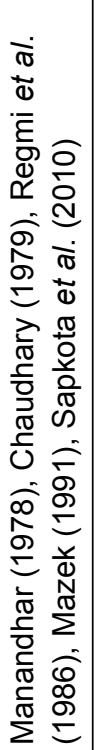 & 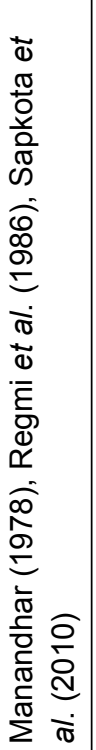 & 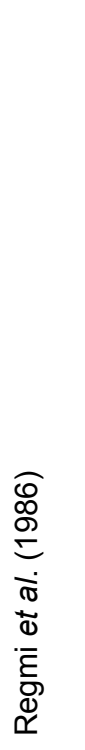 \\
\hline 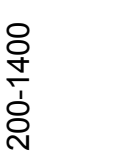 & $\begin{array}{l}\text { 유 } \\
\text { مొ } \\
\text { ’ } \\
\text { مొ } \\
\text { N }\end{array}$ & $\begin{array}{l}\text { ᄋ } \\
\text { ల్ } \\
\text { రి } \\
\text { مె }\end{array}$ & 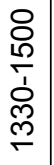 & $\begin{array}{l}\text { ○ी } \\
\text { ભొ } \\
\text { రి } \\
\text { ల్ల }\end{array}$ & 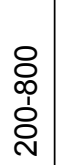 & $\begin{array}{l}\text { ㅇ } \\
\text { స్ } \\
\text { ర্ঠ }\end{array}$ & $\begin{array}{l}8 \\
8 \\
\text { †े } \\
\text { ᄋे } \\
\stackrel{0}{2}\end{array}$ & $\begin{array}{l}\text { 옹 } \\
\frac{1}{\grave{1}} \\
\stackrel{0}{\circ}\end{array}$ & 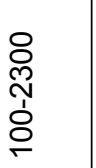 & ద్రి \\
\hline 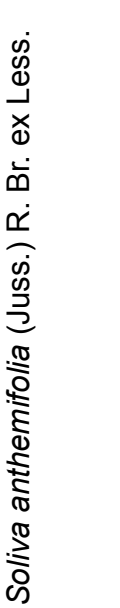 & 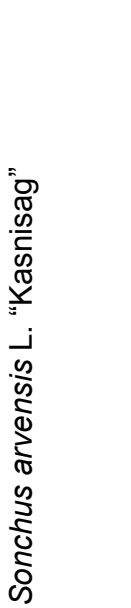 & 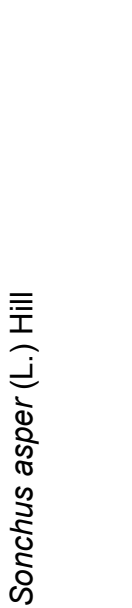 & 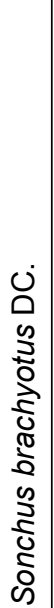 & 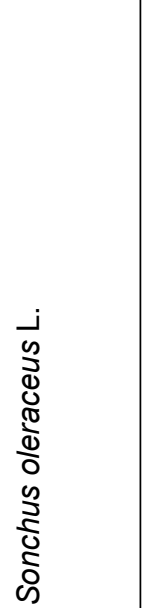 & 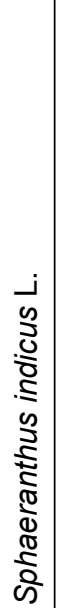 & 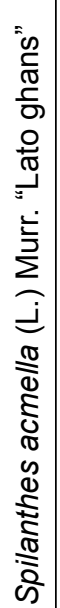 & 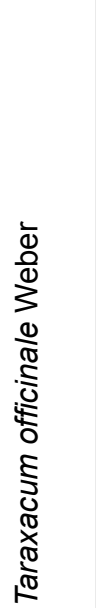 & 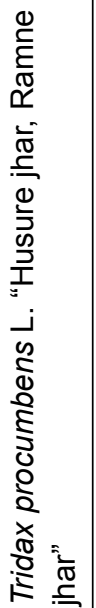 & 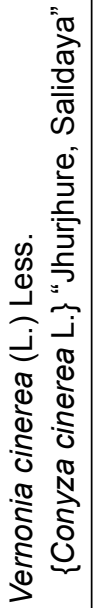 & 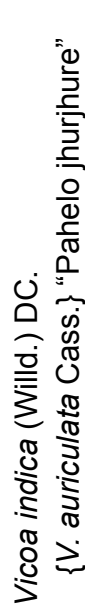 \\
\hline
\end{tabular}




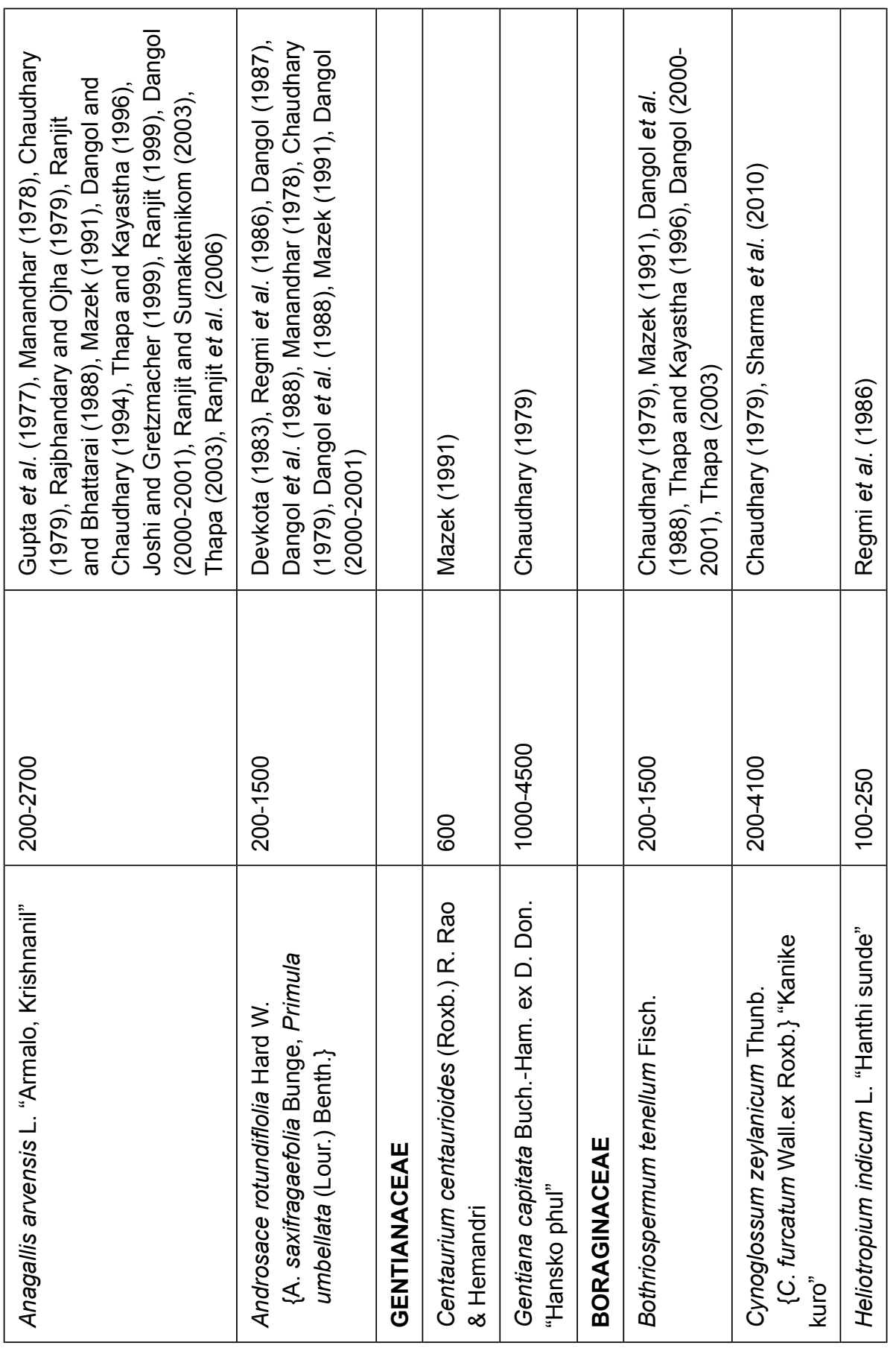




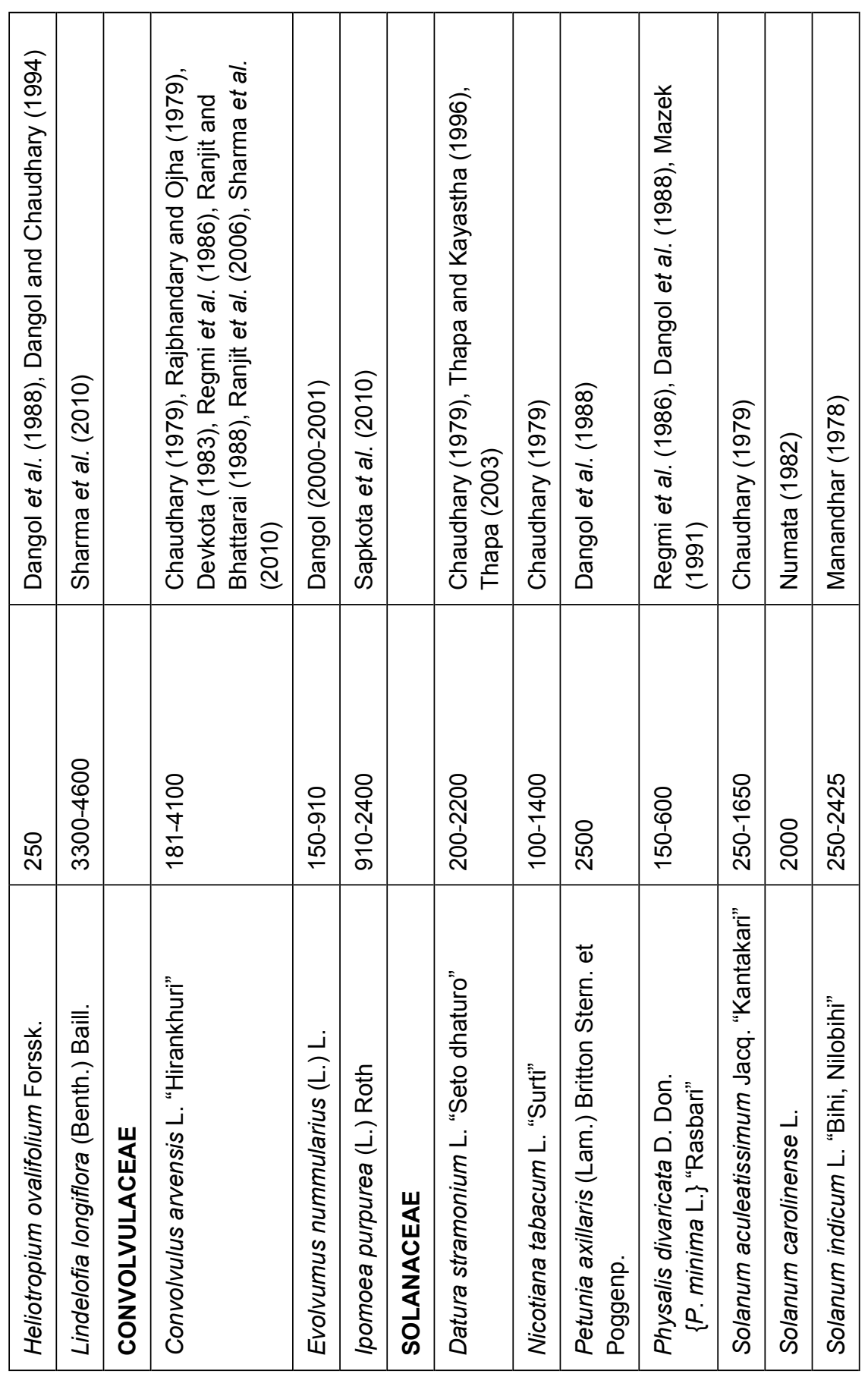




\begin{tabular}{|c|c|c|c|c|c|c|c|c|c|c|}
\hline 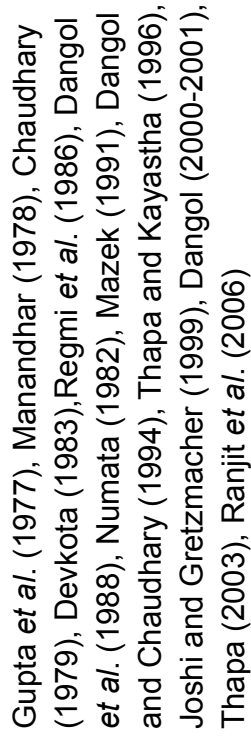 & 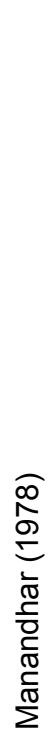 & 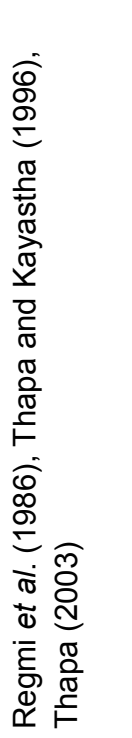 & 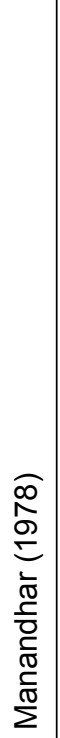 & & 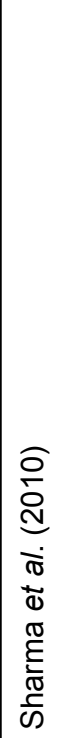 & 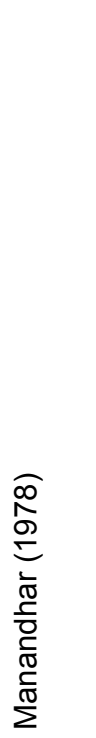 & 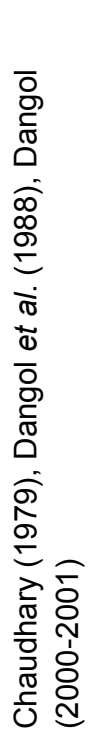 & 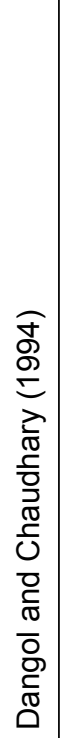 & 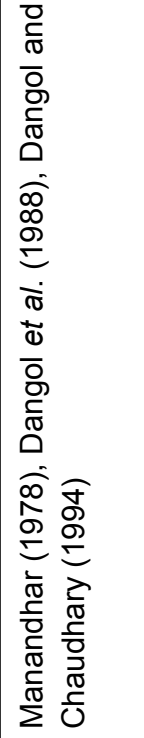 & 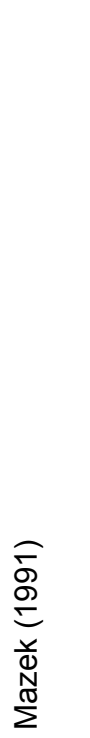 \\
\hline 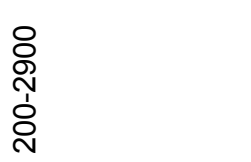 & $\begin{array}{l}\text { ঠి } \\
\text { ஸे } \\
\text { ঠे } \\
\text { † }\end{array}$ & 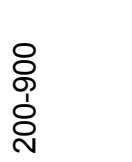 & $\begin{array}{l}8 \\
\text { స్ } \\
\text { ஸे } \\
\stackrel{N}{N}\end{array}$ & & 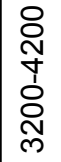 & $\begin{array}{l}8 \\
\text { @ } \\
\text { ஸे } \\
\text { סे }\end{array}$ & 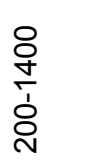 & 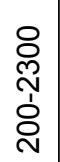 & 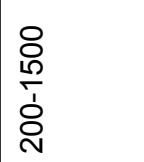 & 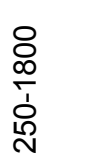 \\
\hline 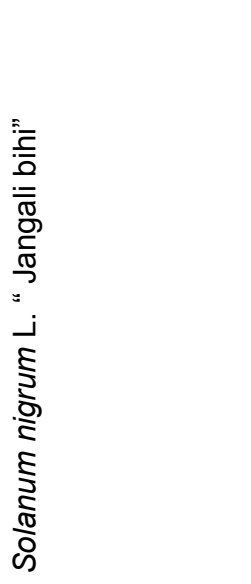 & 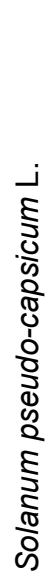 & 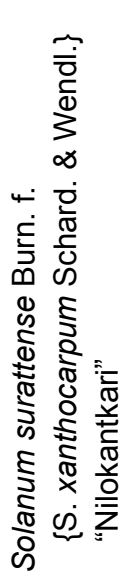 & 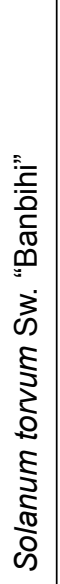 & 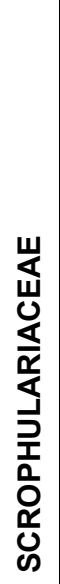 & 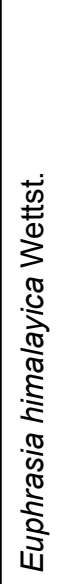 & 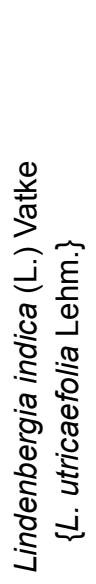 & 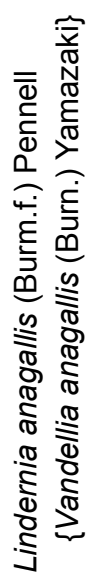 & 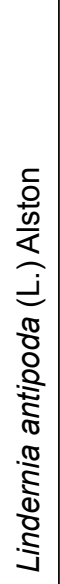 & 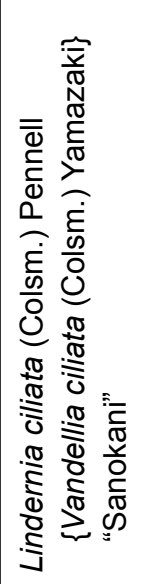 & 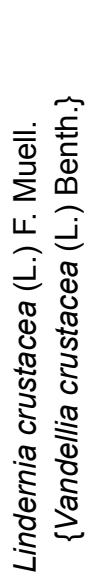 \\
\hline
\end{tabular}




\begin{tabular}{|c|c|c|c|c|c|c|c|c|c|c|c|c|c|}
\hline 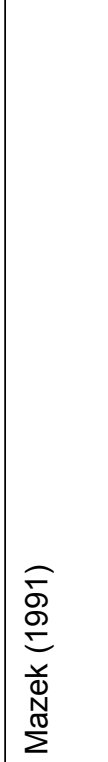 & 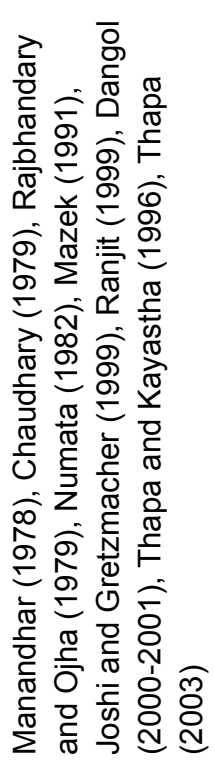 & 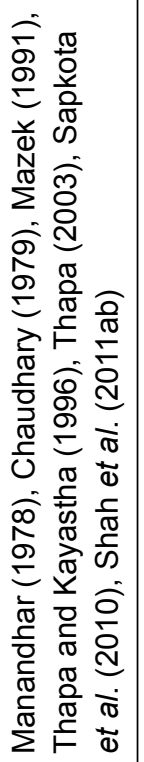 & 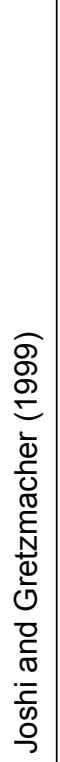 & 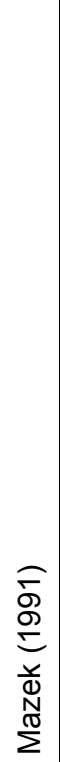 & 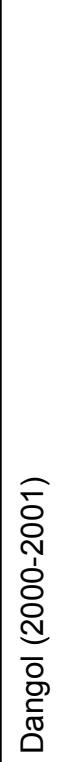 & 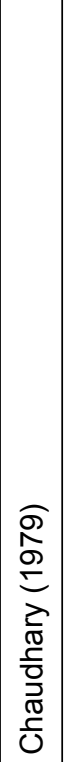 & 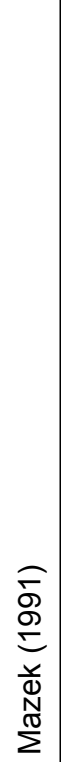 & 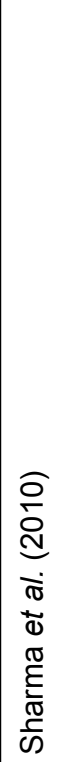 & 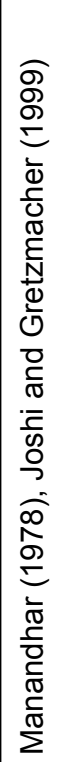 & 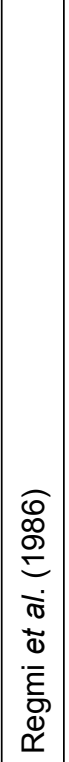 & & 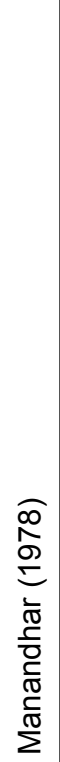 & 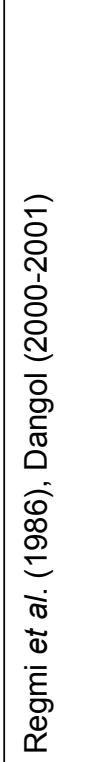 \\
\hline $\begin{array}{l}\text { O্ণ } \\
\text { స̀. } \\
\text { ঠे }\end{array}$ & $\frac{8}{\frac{8}{\grave{~}}}$ & 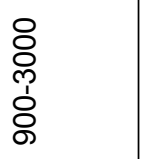 & $\begin{array}{l}\text { 유 } \\
\stackrel{m}{1} \\
\stackrel{ }{D}\end{array}$ & 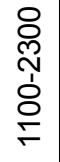 & $\frac{8}{\stackrel{\circ}{\circ}}$ & & 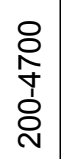 & $\begin{array}{l}\text { ○ } \\
\text { ণ } \\
\text { ல } \\
\text { ల్ }\end{array}$ & 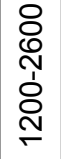 & 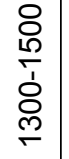 & & 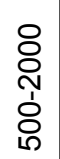 & 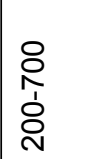 \\
\hline 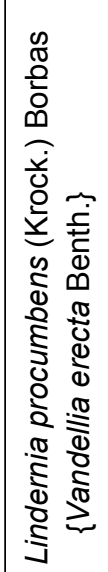 & 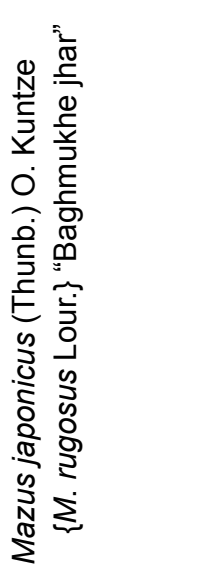 & 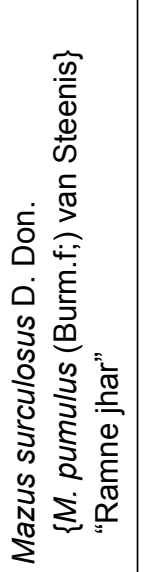 & 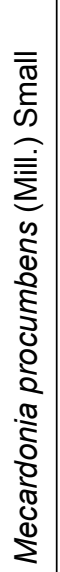 & 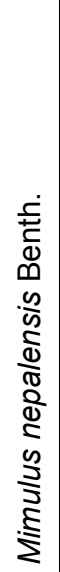 & 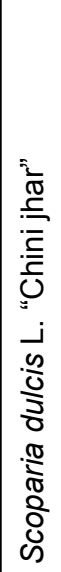 & 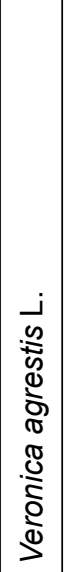 & 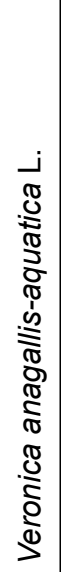 & $\begin{array}{l}-1 \\
0 \\
0 \\
0 \\
0 \\
0 \\
0 \\
.0 \\
0 \\
0 \\
\end{array}$ & 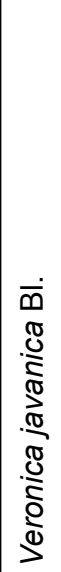 & 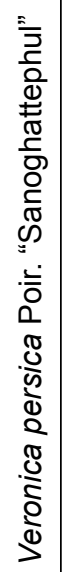 & 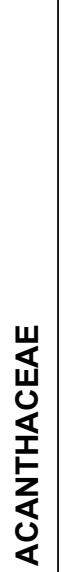 & $\begin{array}{l}0 \\
0 \\
0 \\
2 \\
0 \\
0 \\
00 \\
0 \\
5 \\
0 \\
00 \\
0 \\
0 \\
00 \\
000 \\
000 \\
0.0\end{array}$ & 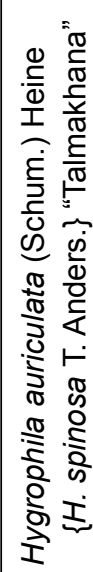 \\
\hline
\end{tabular}




\begin{tabular}{|c|c|c|c|c|c|c|c|c|c|c|c|c|}
\hline 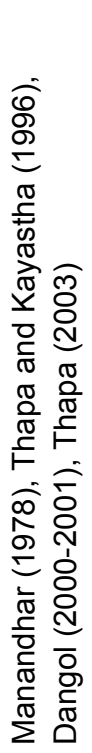 & 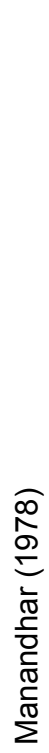 & 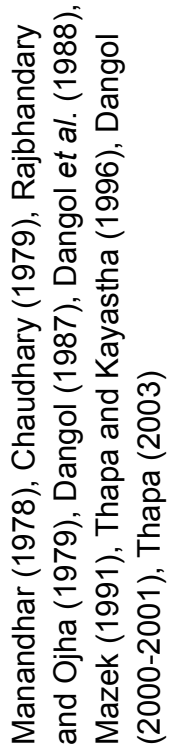 & & 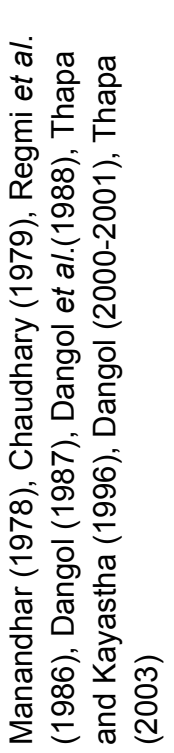 & 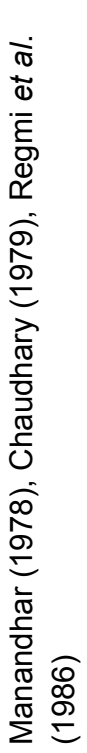 & & 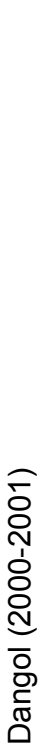 & 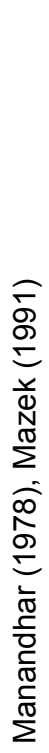 & 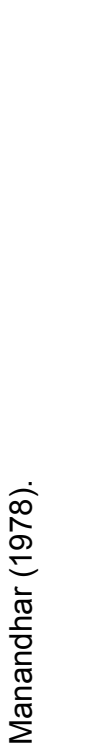 & 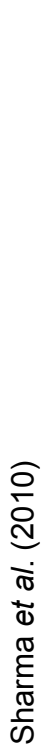 & 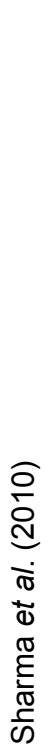 & 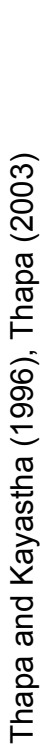 \\
\hline 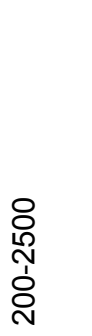 & $\begin{array}{l}\text { 음 } \\
\text { ஸे } \\
\text { 음 }\end{array}$ & 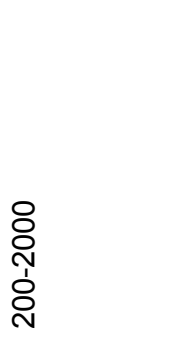 & & $\frac{\text { ᄋ }}{\frac{+}{+}}$ & 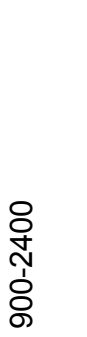 & & 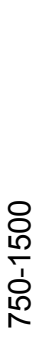 & 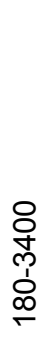 & 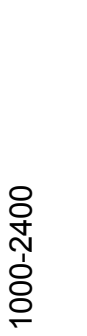 & & 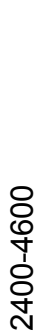 & ஓి \\
\hline 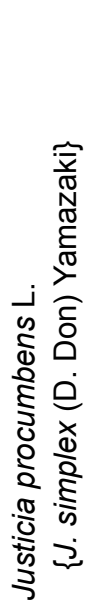 & 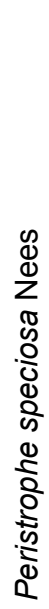 & 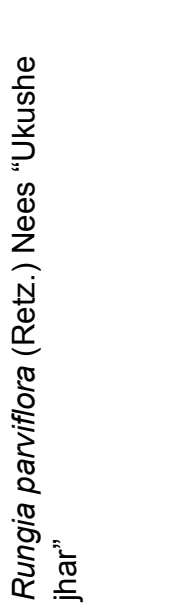 & 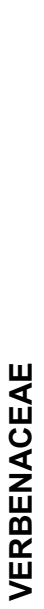 & 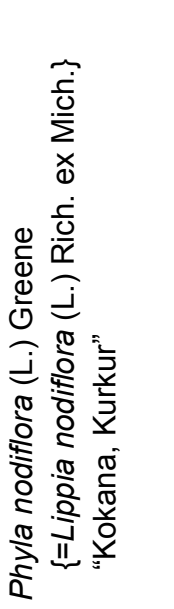 & 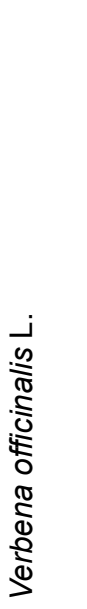 & 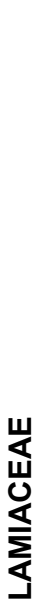 & 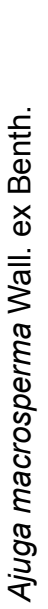 & 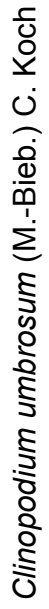 & 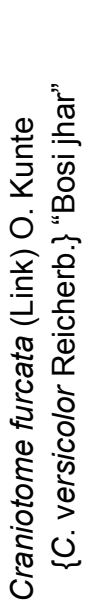 & 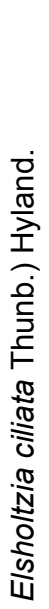 & 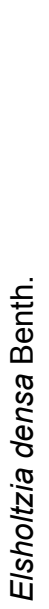 & 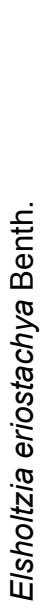 \\
\hline
\end{tabular}




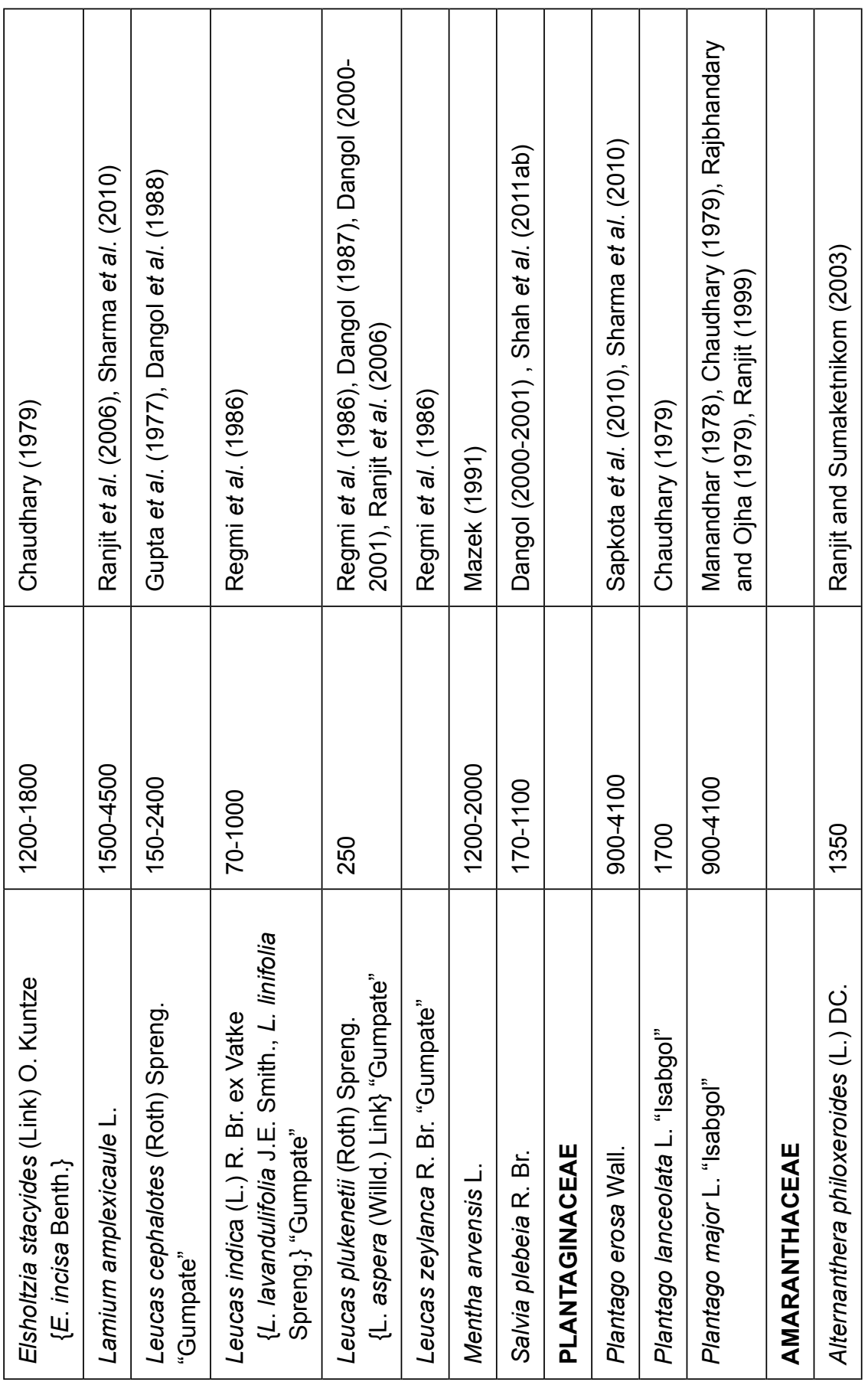




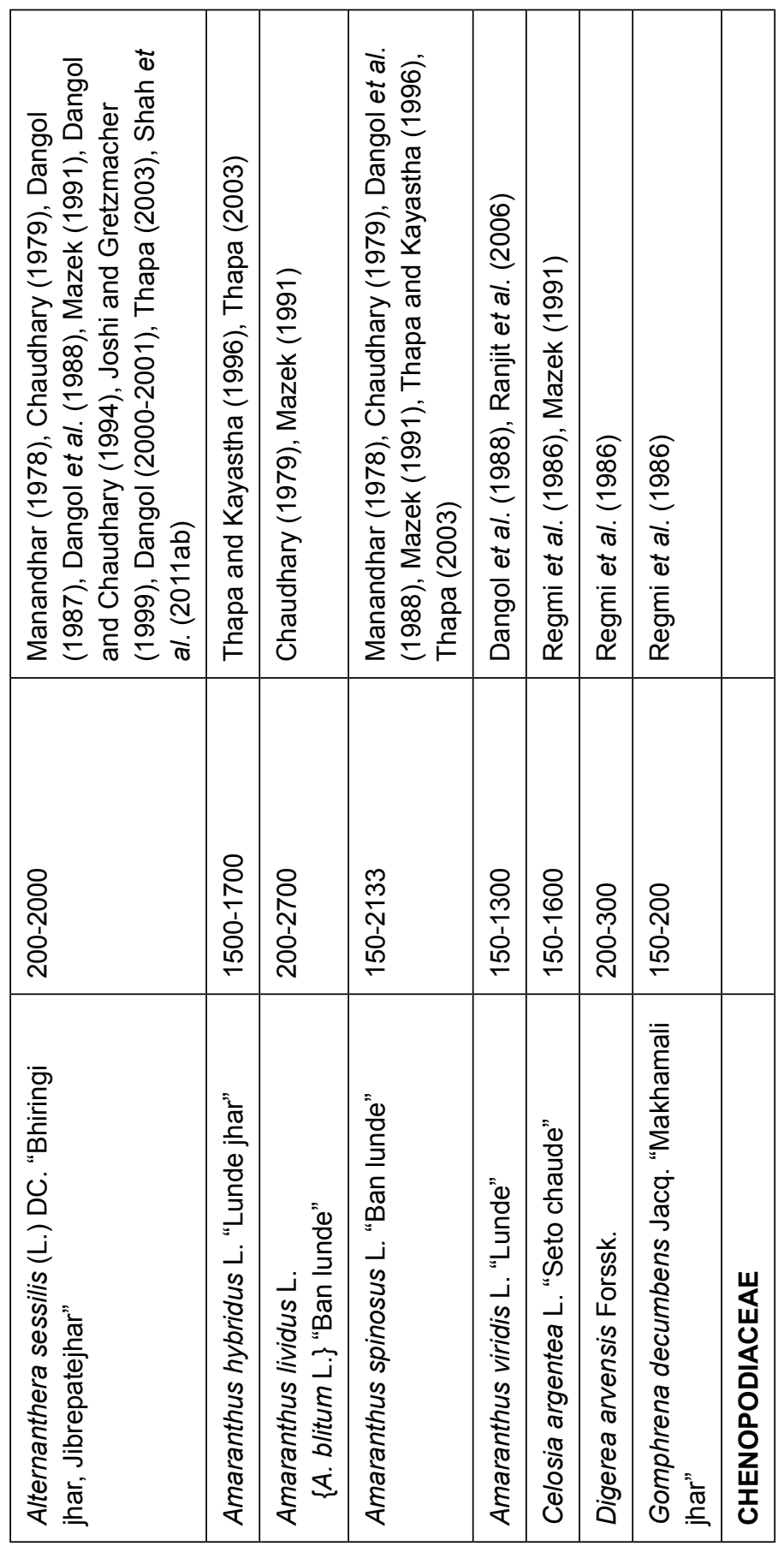




\begin{tabular}{|c|c|c|c|c|c|c|c|}
\hline 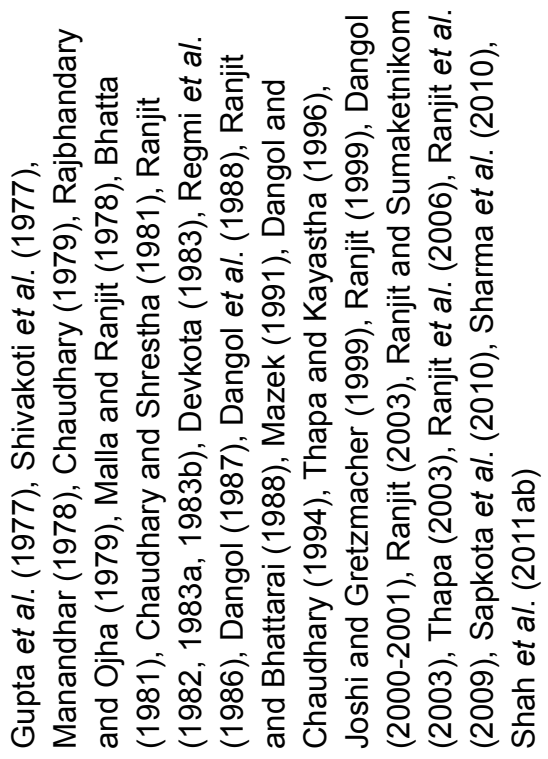 & 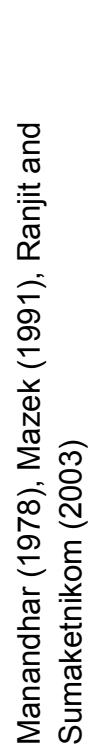 & 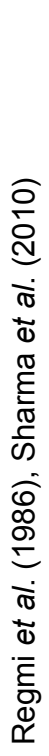 & 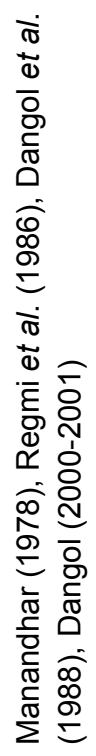 & & 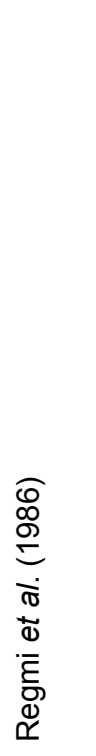 & 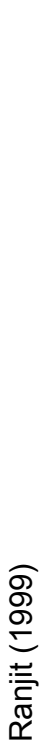 & 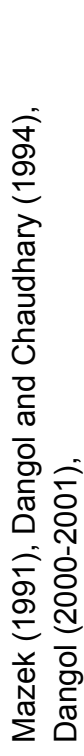 \\
\hline $\begin{array}{l}8 \\
\text { ○ } \\
\text { †े } \\
\text { ঠ̊ }\end{array}$ & $\begin{array}{l}\text { ᄋి } \\
\text { ల్ } \\
\text { రి } \\
\text { }\end{array}$ & $\begin{array}{l}\text { ᄋ } \\
\text { ㅇ } \\
\text { ᄋ } \\
\text { ঠे }\end{array}$ & 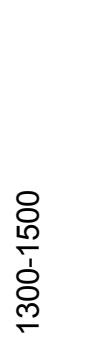 & & 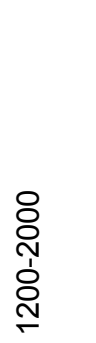 & 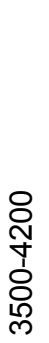 & 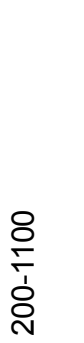 \\
\hline 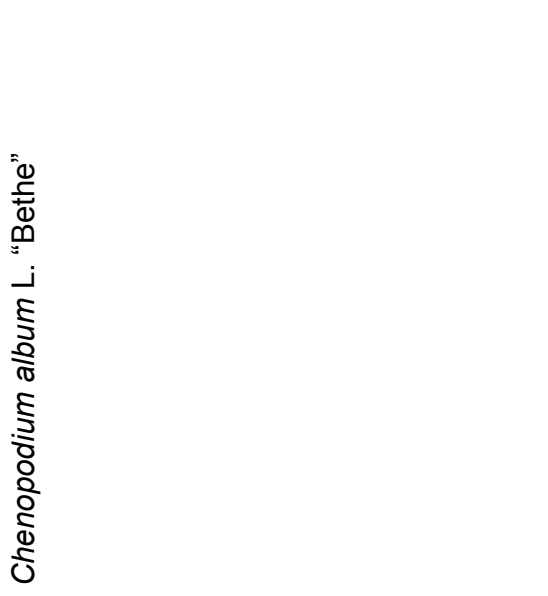 & 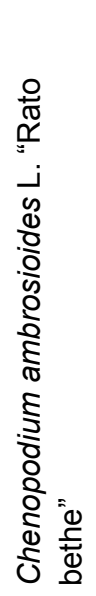 & 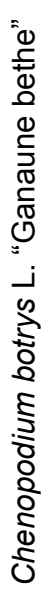 & 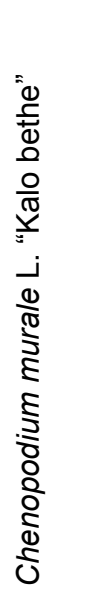 & 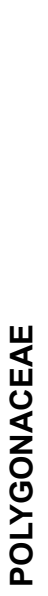 & 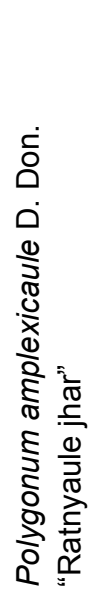 & 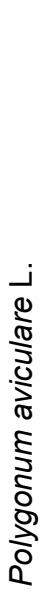 & 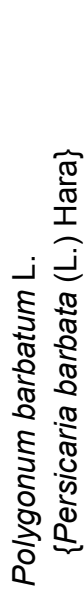 \\
\hline
\end{tabular}




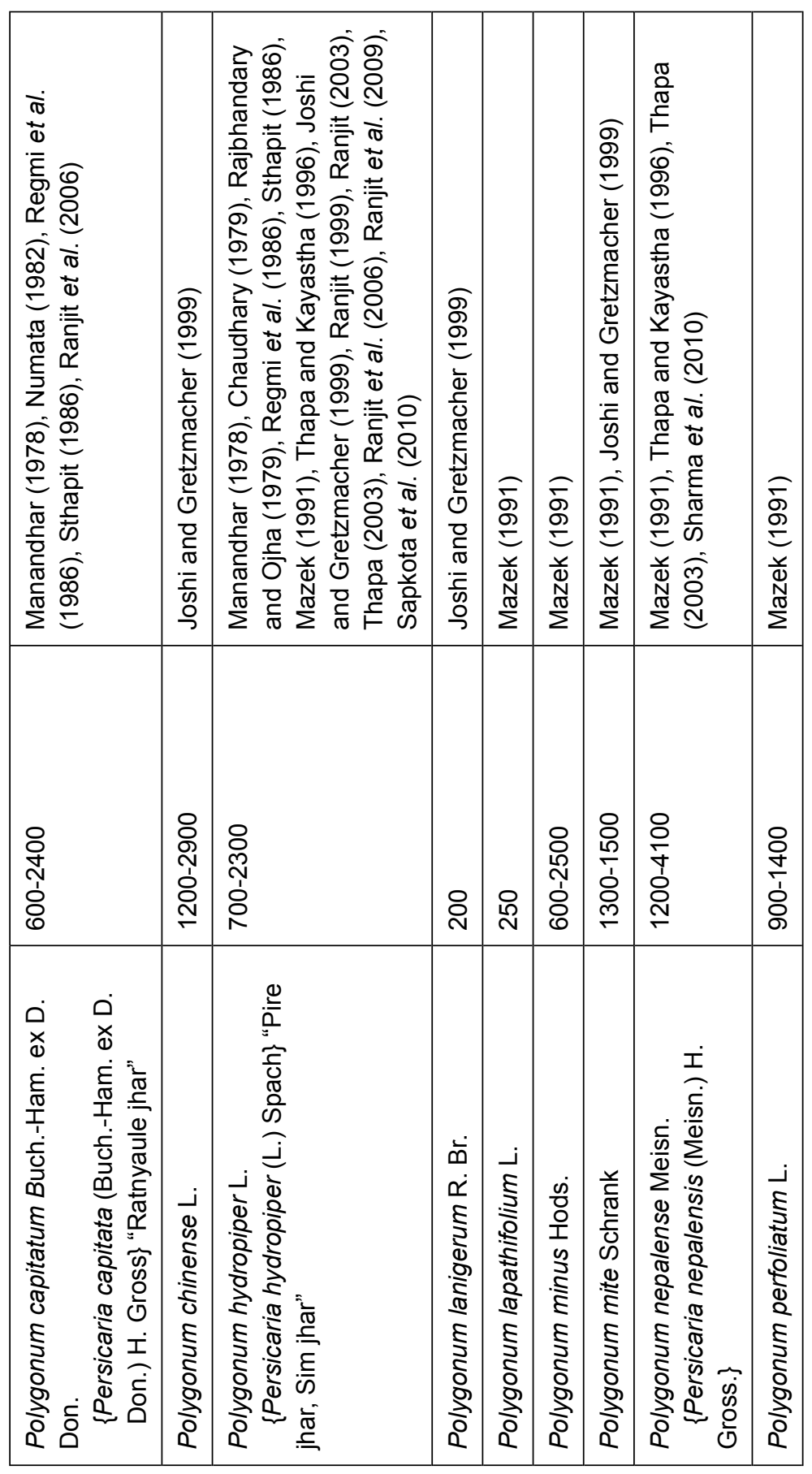




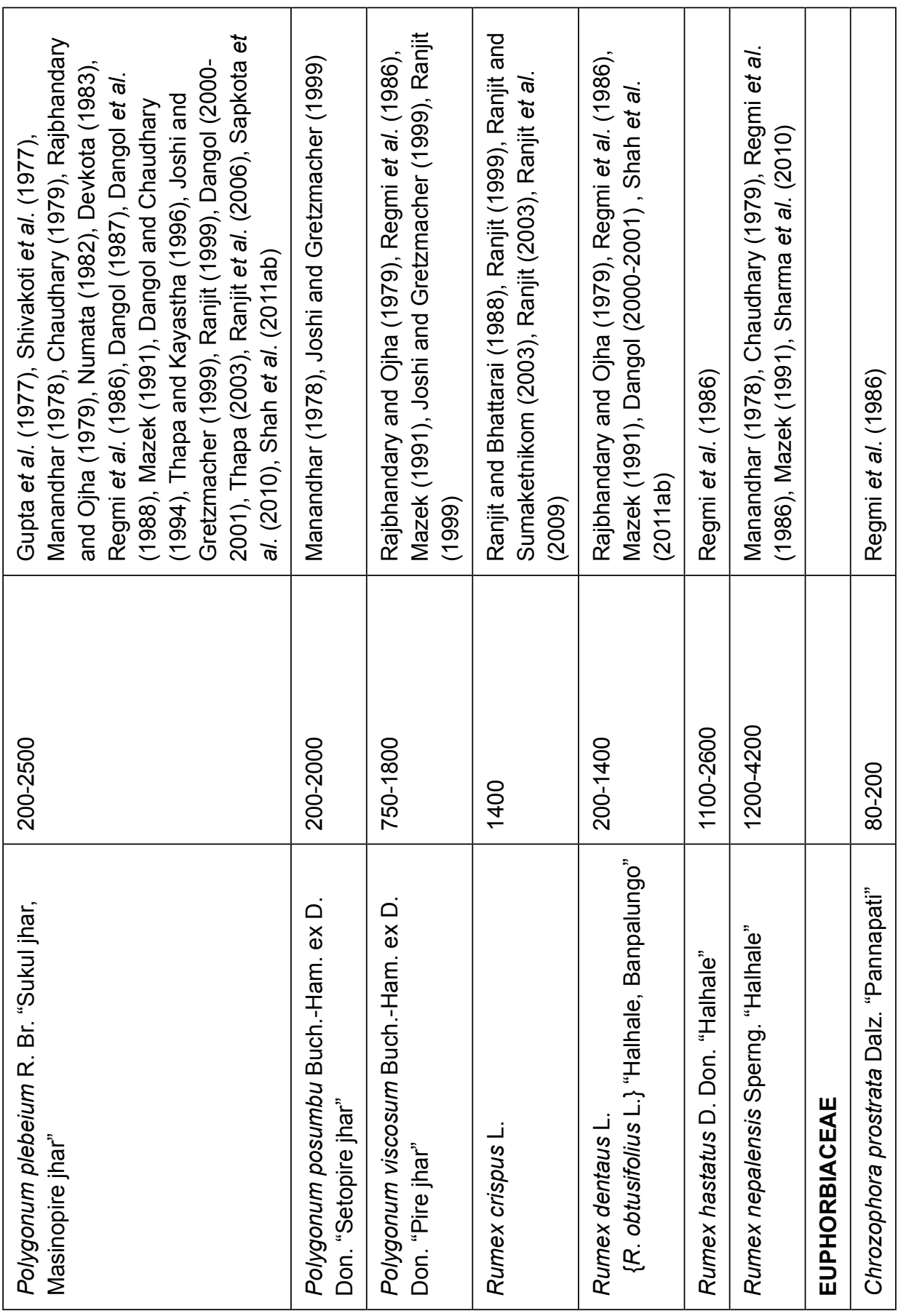




\begin{tabular}{|c|c|c|c|c|c|c|c|c|c|c|c|}
\hline 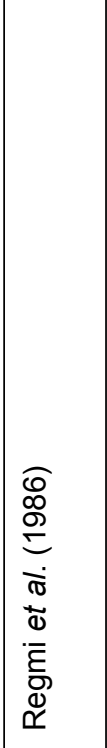 & 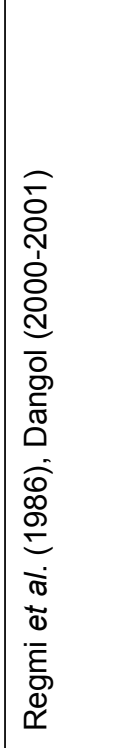 & 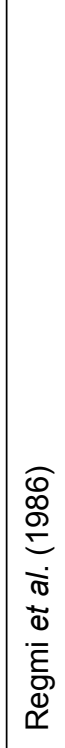 & 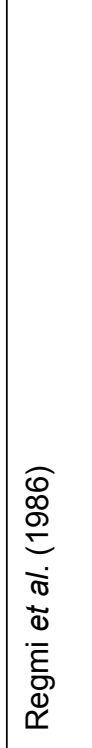 & 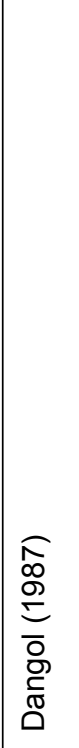 & 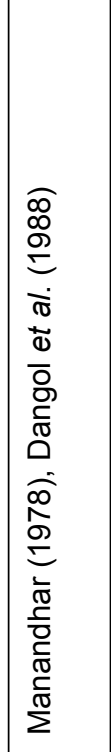 & 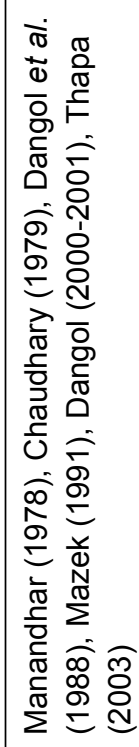 & 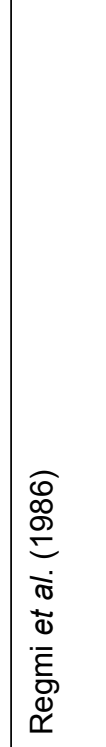 & 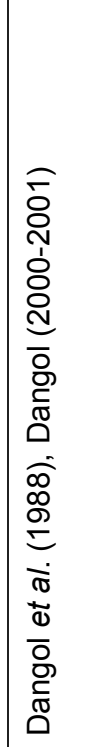 & 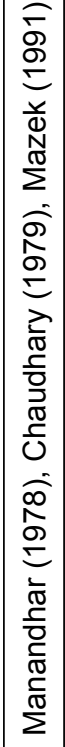 & 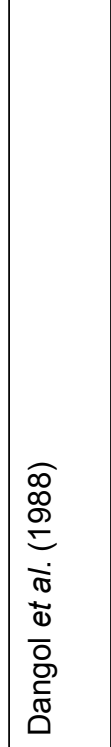 & 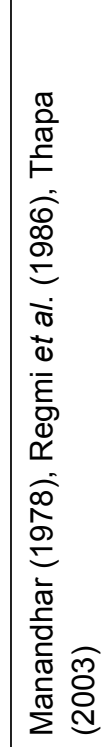 \\
\hline 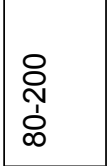 & $\begin{array}{l}\text { o } \\
\text { ô } \\
\text { o } \\
0\end{array}$ & $\begin{array}{l}8 \\
0 \\
1 \\
1\end{array}$ & 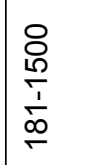 & $\begin{array}{l}\text { Oి } \\
\text { o } \\
\stackrel{0}{0} \\
\stackrel{2}{3}\end{array}$ & 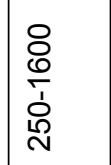 & $\mid \begin{array}{l}8 \\
0 \\
0 \\
0\end{array}$ & & \begin{tabular}{|l} 
ơ \\
ț \\
ph
\end{tabular} & 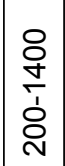 & 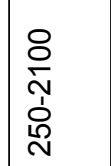 & 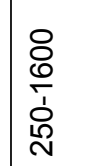 \\
\hline 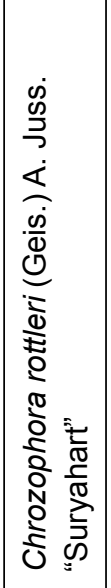 & 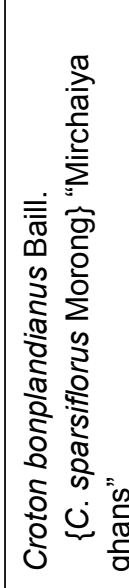 & 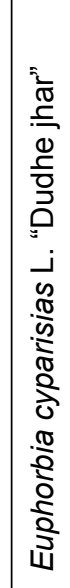 & 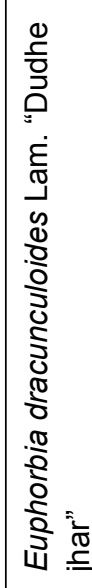 & 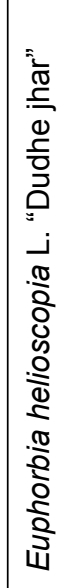 & 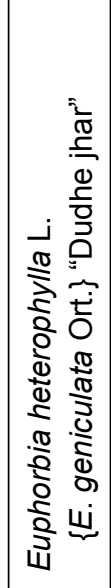 & 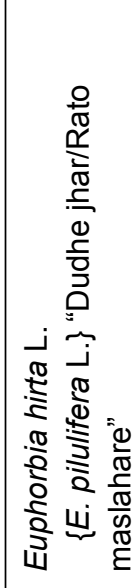 & 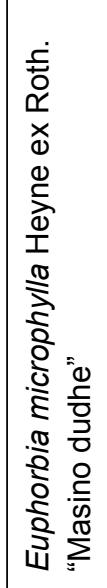 & 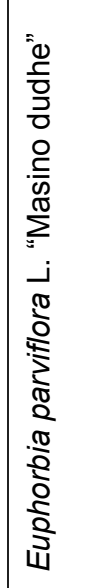 & 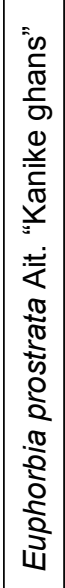 & 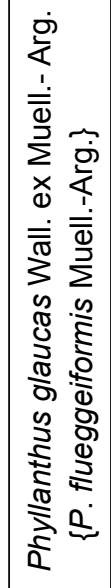 & 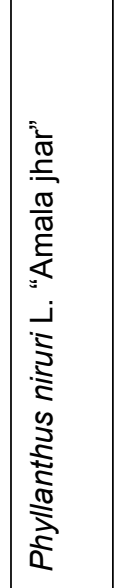 \\
\hline
\end{tabular}




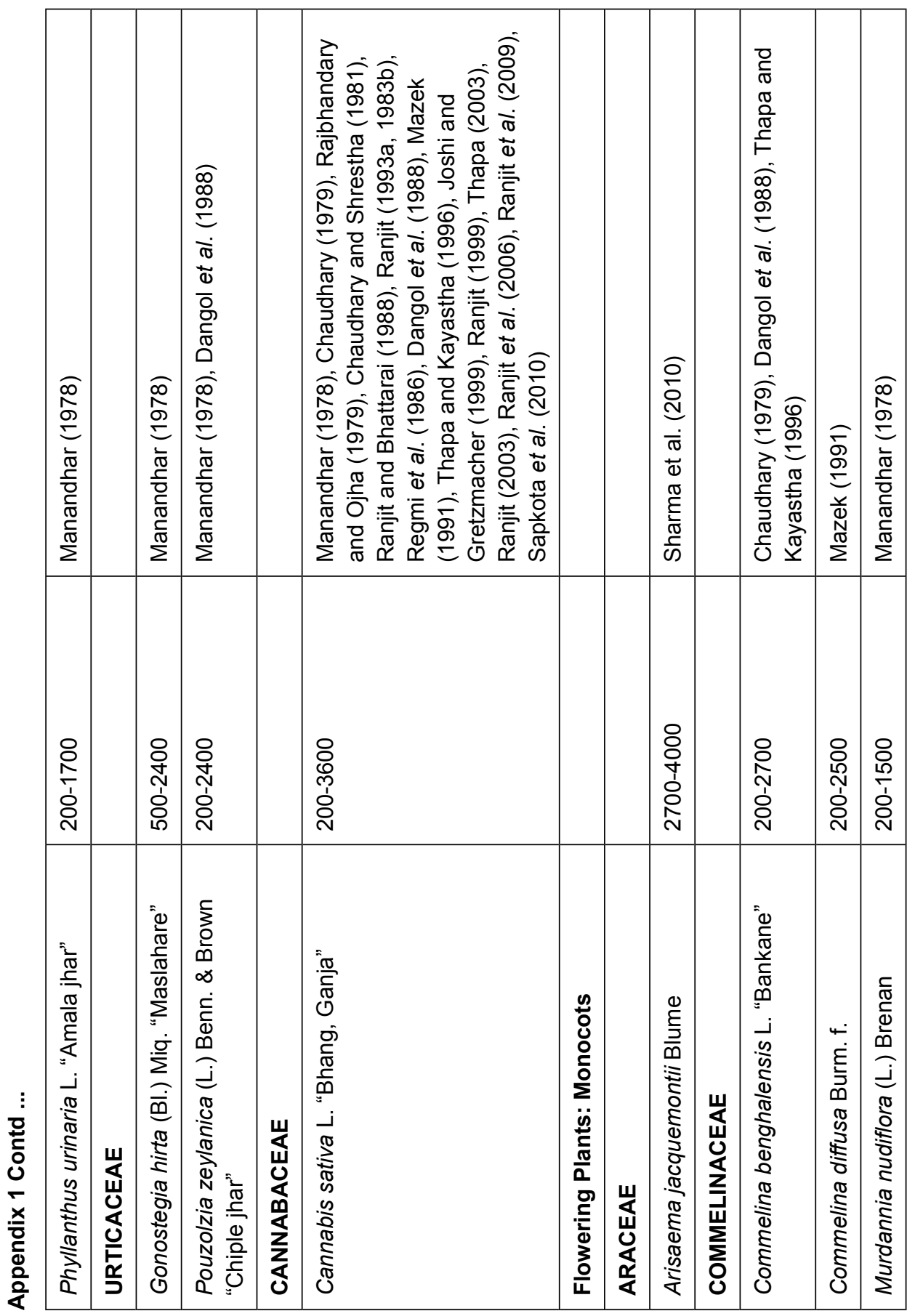




\begin{tabular}{|c|c|c|c|c|c|c|c|c|c|c|c|c|c|c|c|}
\hline & 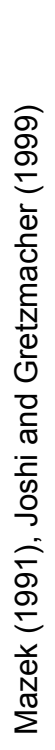 & 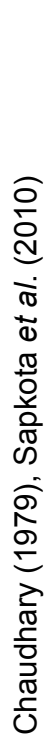 & 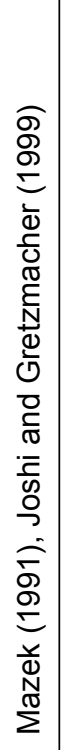 & & 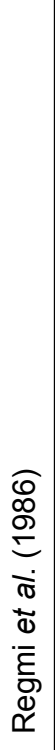 & & 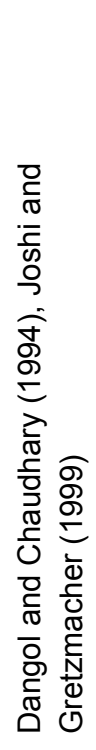 & 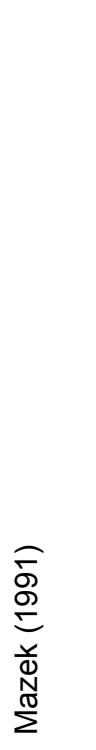 & 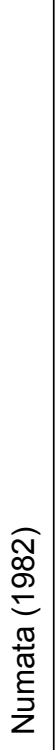 & 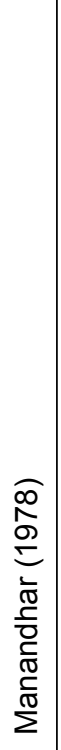 & 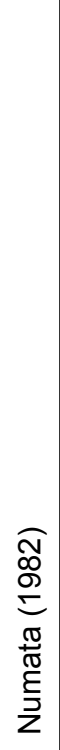 & 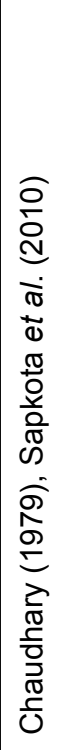 & 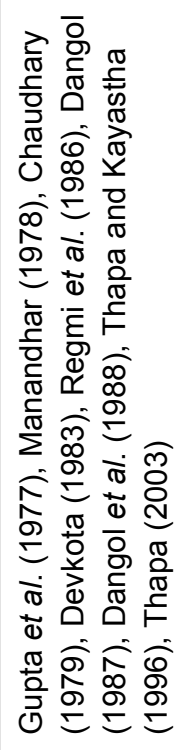 & 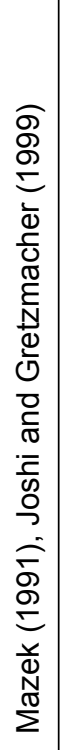 & 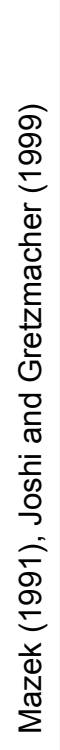 \\
\hline & 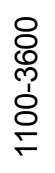 & 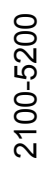 & 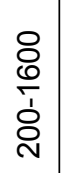 & & 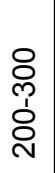 & & 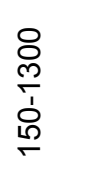 & 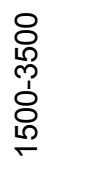 & $\begin{array}{l}8 \\
8 \\
+ \\
8 \\
8 \\
10 \\
\end{array}$ & 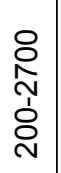 & $\begin{array}{l}\text { ᄋ } \\
\text { స్ } \\
\text { ○े } \\
\stackrel{N}{N}\end{array}$ & 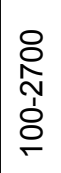 & \begin{tabular}{l} 
O \\
\multirow{+}{+}{} \\
$\frac{1}{\infty}$ \\
$\stackrel{\infty}{-}$
\end{tabular} & 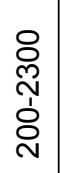 & 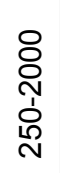 \\
\hline 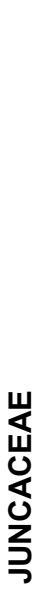 & 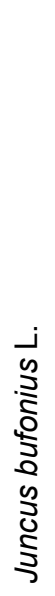 & 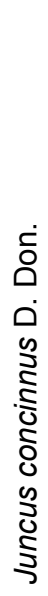 & 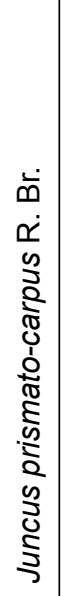 & 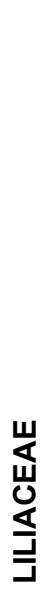 & 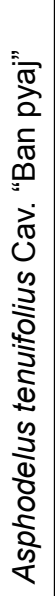 & 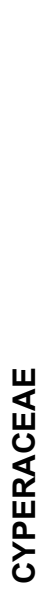 & 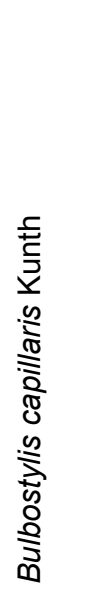 & 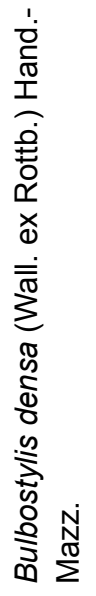 & 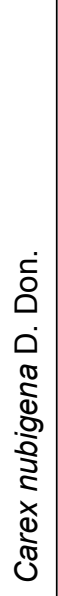 & 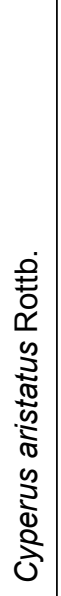 & 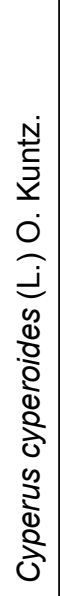 & 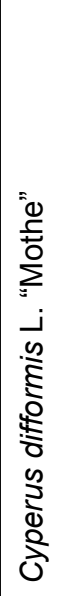 & 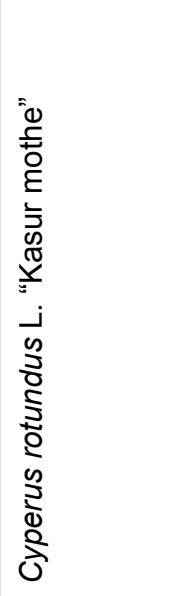 & 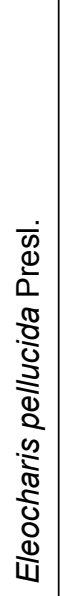 & 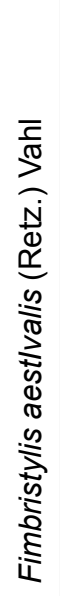 \\
\hline
\end{tabular}




\begin{tabular}{|c|c|c|c|c|c|c|c|c|c|c|c|c|}
\hline 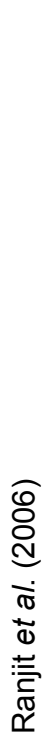 & 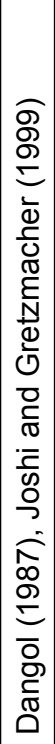 & 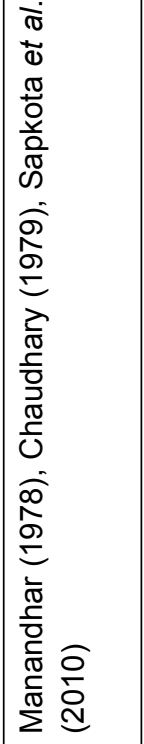 & 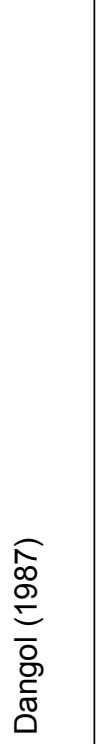 & & 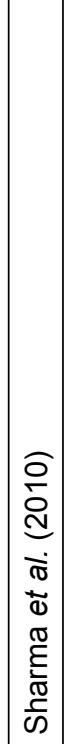 & 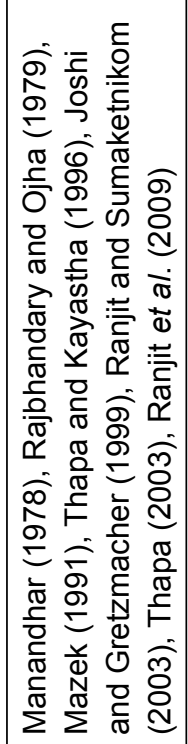 & 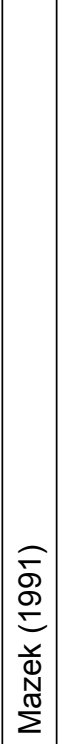 & 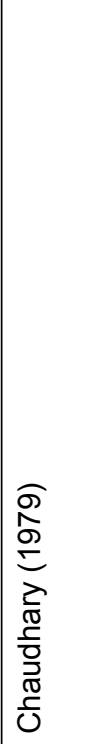 & 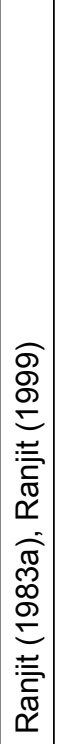 & 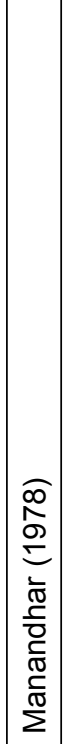 & 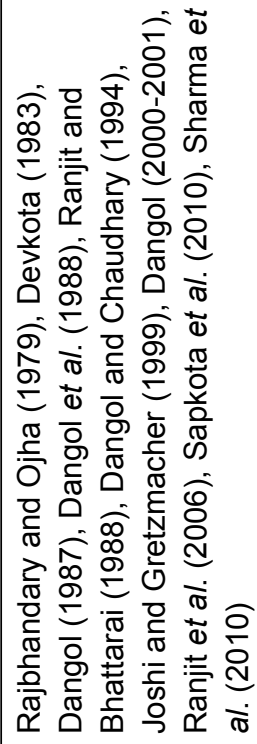 & 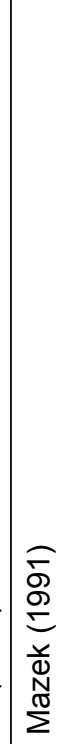 \\
\hline 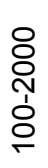 & 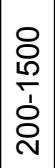 & $\begin{array}{l}\text { ㅇ } \\
\text { ஸે } \\
\text { ○े } \\
\text { ᄋ }\end{array}$ & 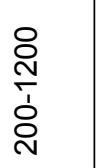 & & 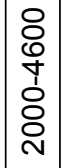 & $\begin{array}{l}\text { O্ } \\
\text { N } \\
\text { ঠे } \\
\text { ల్ల }\end{array}$ & $\stackrel{8}{\stackrel{8}{2}}$ & \& & $\begin{array}{l}8 \\
8 \\
\square\end{array}$ & 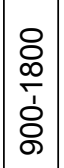 & 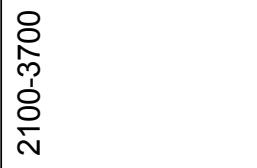 & $\begin{array}{l}\text { ᄋ } \\
\frac{\infty}{\grave{1}} \\
\text { ঠे }\end{array}$ \\
\hline 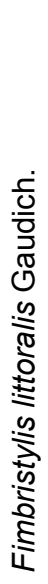 & 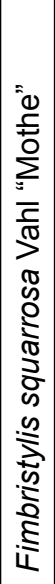 & 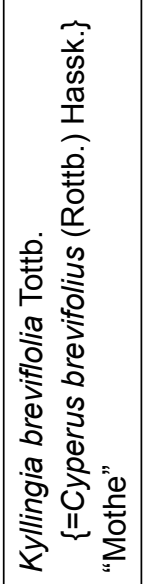 & 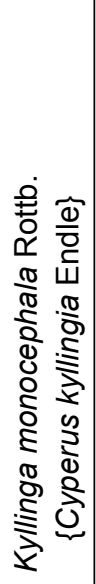 & 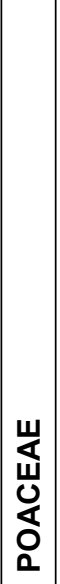 & 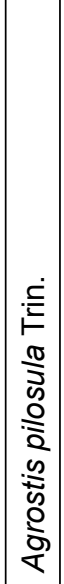 & 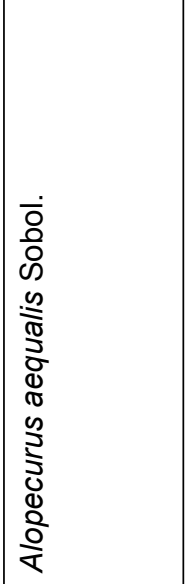 & 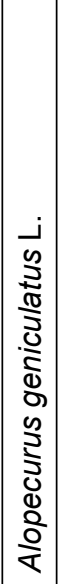 & 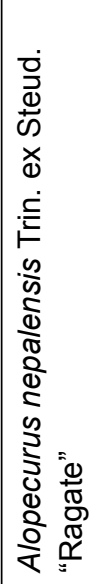 & 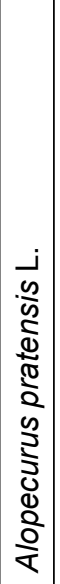 & 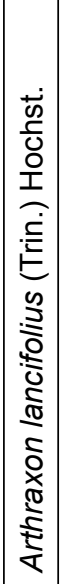 & 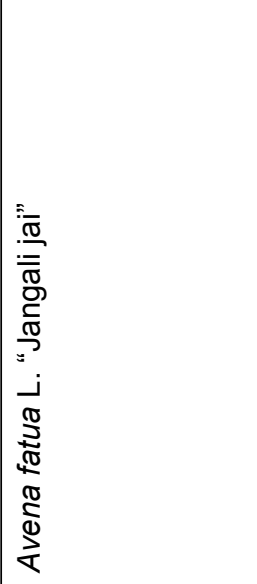 & 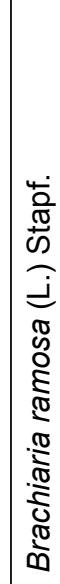 \\
\hline
\end{tabular}




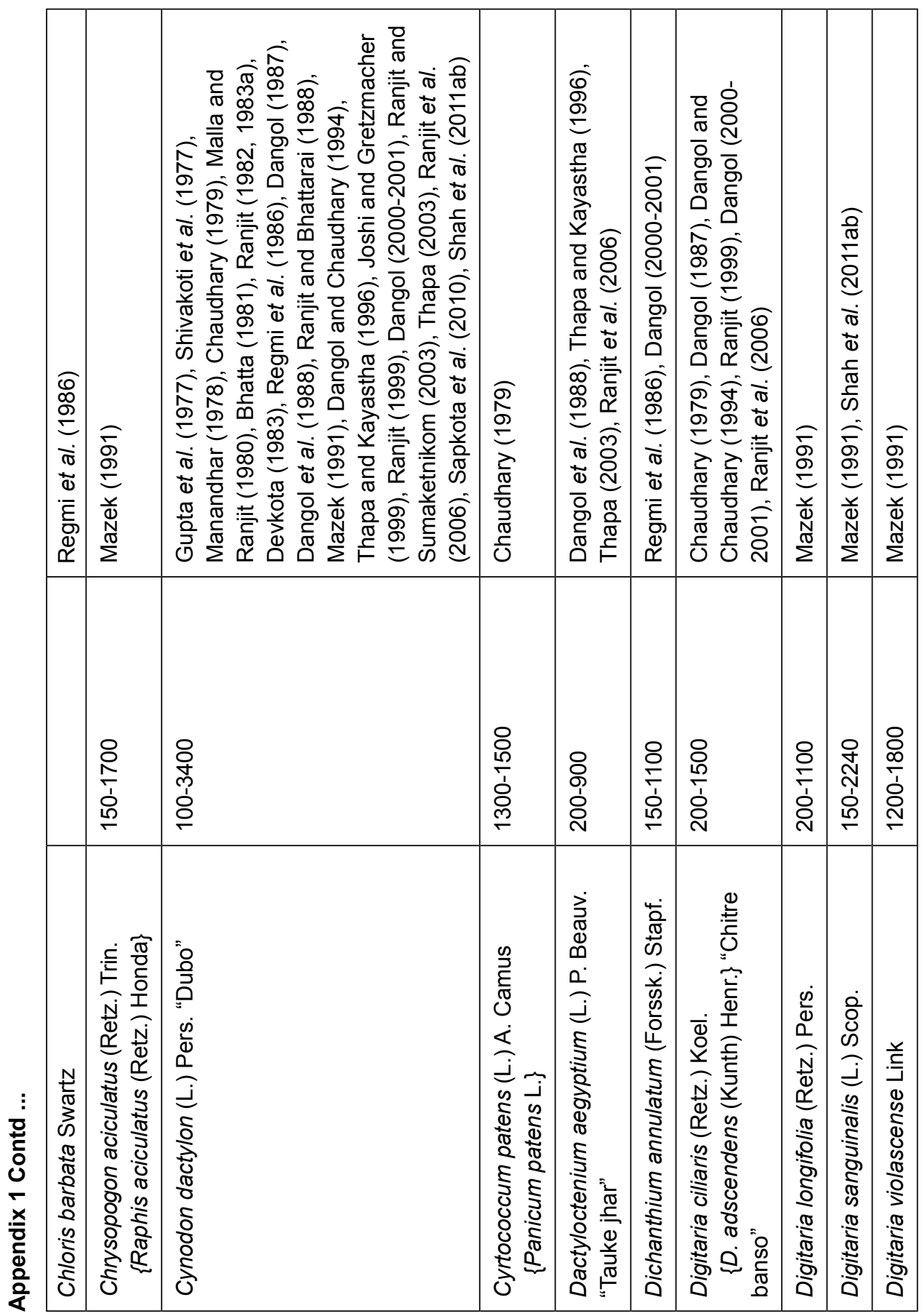




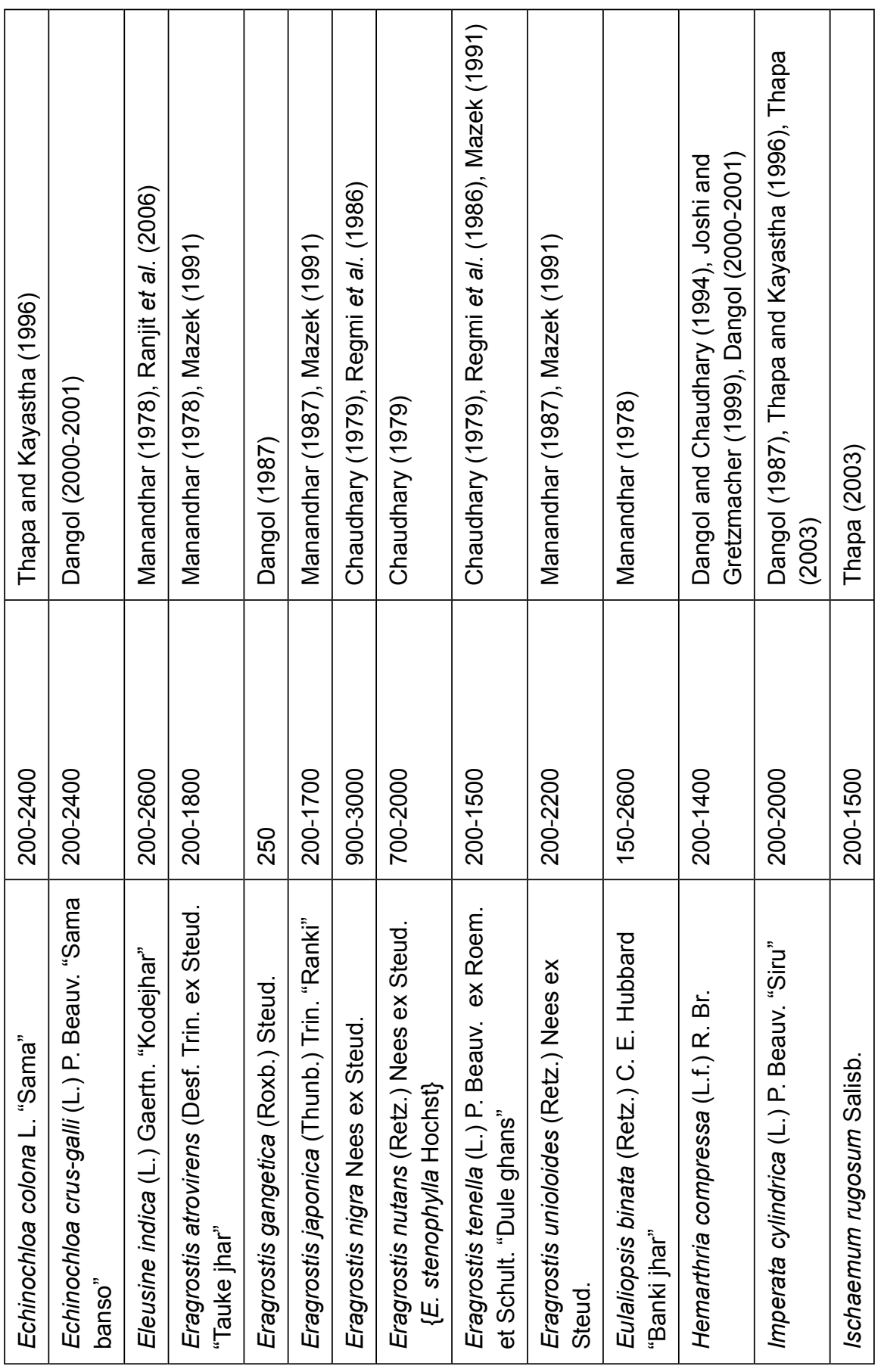




\begin{tabular}{|c|c|c|c|c|c|c|c|c|}
\hline 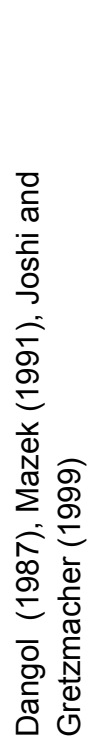 & 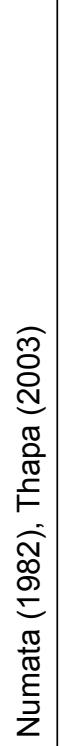 & 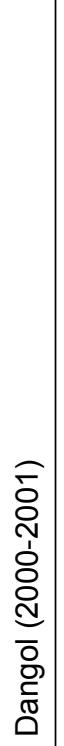 & 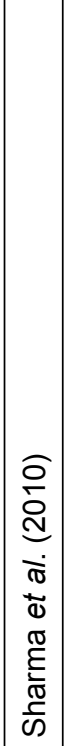 & 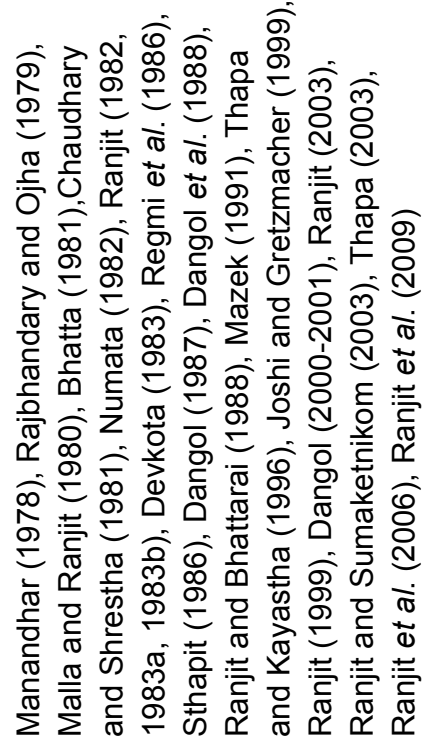 & 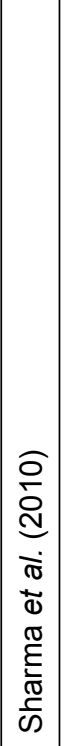 & 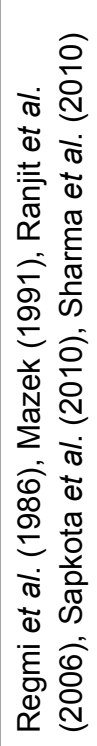 & 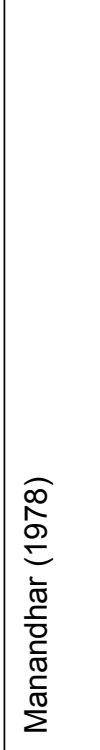 & 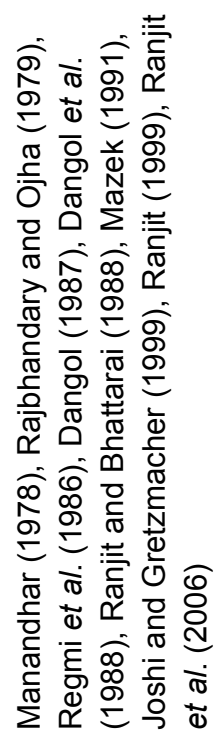 \\
\hline $\begin{array}{l}8 \\
\frac{1}{1} \\
\frac{1}{8}\end{array}$ & 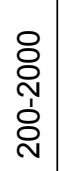 & 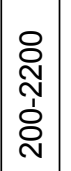 & 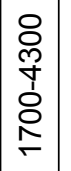 & 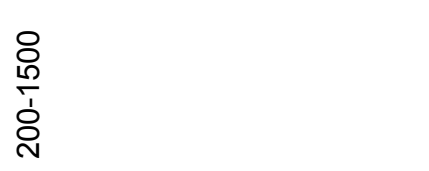 & & 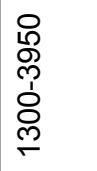 & 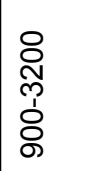 & 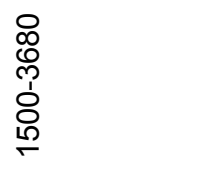 \\
\hline 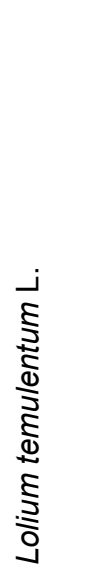 & 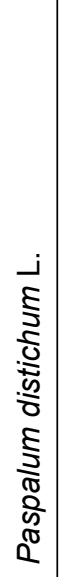 & 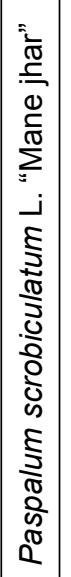 & 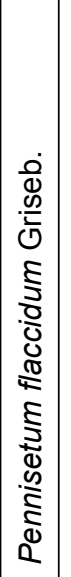 & 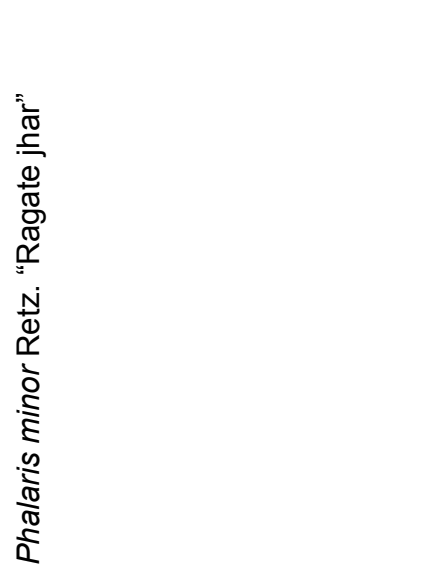 & 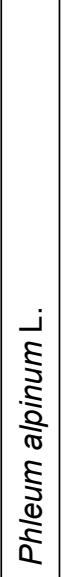 & 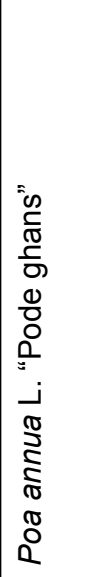 & 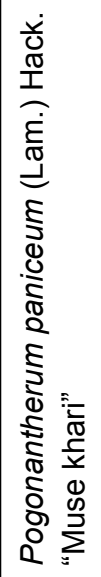 & 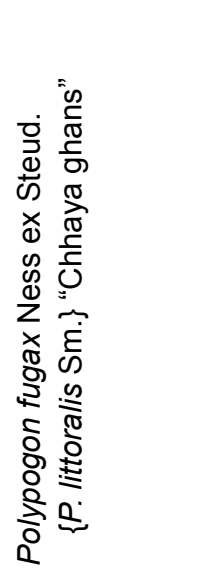 \\
\hline
\end{tabular}




\begin{tabular}{|c|c|c|c|c|c|c|c|c|c|c|}
\hline 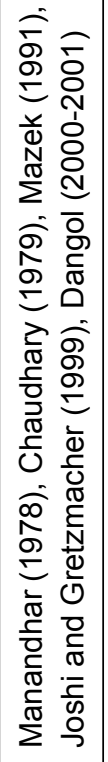 & 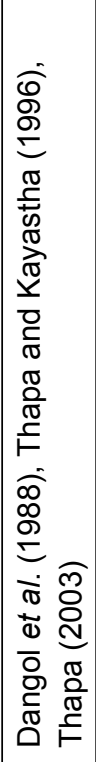 & 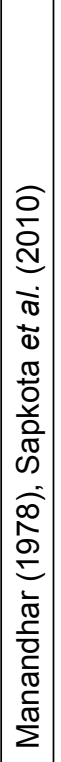 & 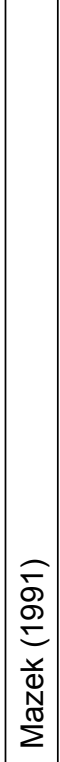 & 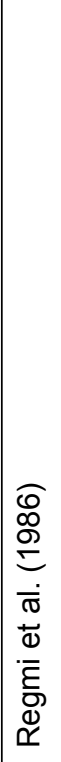 & 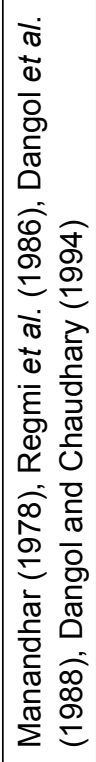 & 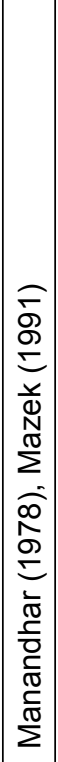 & 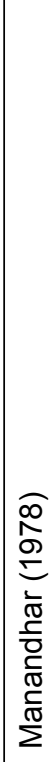 & 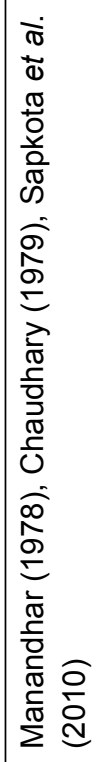 & 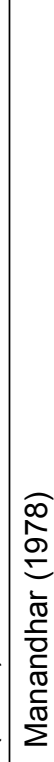 & 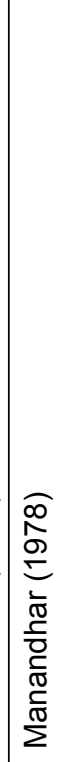 \\
\hline
\end{tabular}

ำ

$\infty$

ลิ

बं

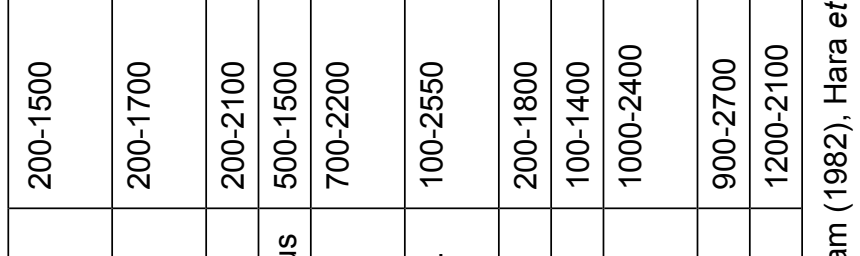

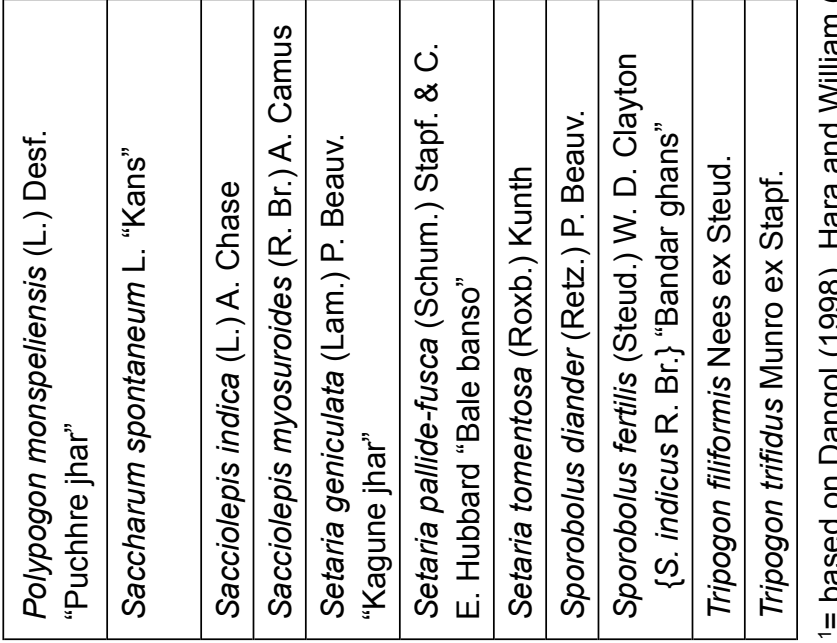

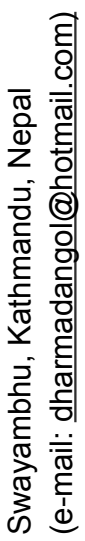

